\title{
Shells with Hybrid Active-Passive Damping Treatments: Modeling and Vibration Control
}

\author{
C. M. A. Vasques* and J. Dias Rodrigues ${ }^{\dagger}$ \\ Faculdade de Engenharia da Universidade do Porto, Porto, Portugal
}

\begin{abstract}
This paper concerns the mathematical modeling and finite element (FE) solution of general anisotropic shells with hybrid active-passive damping treatments. A fully-coupled piezo-visco-elastic mathematical model of the shell (host structure) and segmented arbitrarily stacked layers of damping treatments is considered. A discrete layer approach is employed in this work, and the weak form of the governing equations is derived for a single generic layer of the multilayer shell using Hamilton's principle and a mixed (displacement/stresses) definition of the displacement field. First, a fully refined deformation theory of the generic layer, based on postulated out-of-plane shear stress definitions and in the in-plane stresses obtained with a Reissner-Mindlin type shell theory, is outlined. A semi-inverse procedure is used to derive the layer mixed non-linear displacement field, in terms of a blend of the generalized displacements of the Love-Kirchhoff and ReissnerMindlin theories and of the stress components at the generic layer interfaces. No assumptions regarding the thinness of the shell are considered. Regarding the definition of the electric potential, the direct piezoelectric effects are condensed into the model through effective stiffness and strains definitions, and the converse counterpart is considered by the action of prescribed electric potential differences in each piezoelectric layer. Then, the weak forms of a partially refined theory, where only the zero-order term of the non-linear fully refined transverse displacement is retained, are derived for an orthotropic doublycurved piezo-elastic generic shell layer. Based on the weak forms a FE solution is initially developed for the single layer. The degrees of freedom (DoFs) of the resultant four-noded generic piezo-elastic single layer FE are then "regenerated" into an equivalent eight-node 3-D formulation in order to allow through-the-thickness assemblage of displacements and stresses, yielding a partially refined multilayer FE assuring displacement and shear stress interlayer continuity and homogeneous shear stress conditions at the outer surfaces. The shear stresses DoFs are dynamically condensed and the FE is reduced to a displacementbased form. The viscoelastic damping behavior is considered at the global FE model level by means of a Laplace transformed ADF model. The active control of vibration is shortly discussed and a set of indices to quantify the damping performance and the individual contributions of the different mechanisms are proposed.
\end{abstract}

\section{Introduction}

$\mathrm{T}$ HE 21st century has emerged with a "new" challenge for science. Unified multiphysics coupled formulations moving towards the precise modeling of real physical problem pushes the underlying complexity to higher levels. It involves the efforts of specialists of different areas contributing in a unified way to find a solution to those "complex" coupled problems. In parallel with that demanding refinement of the models, the processing capabilities of modern computers has also increased. An example of that multidisciplinarity can be found in the modeling of general plane or curved structures with hybrid active-passive damping treatments which are applied to control structural vibrations and/or noise radiation from structures.

*PhD Student, Faculdade de Engenharia da Universidade do Porto, Departamento de Engenharia Mecânica e Gestão Industrial, Rua Dr. Roberto Frias s/n, Edifício M, Sala 206, 4200-465 Porto, Portugal, AIAA Student Member.

${ }^{\dagger}$ Associate Professor, Faculdade de Engenharia da Universidade do Porto, Departamento de Engenharia Mecânica e Gestão Industrial, Rua Dr. Roberto Frias s/n, 4200-465 Porto, Portugal. 
In order to efficiently model these treatments, the piezo-visco-elastic couplings between an elastic host structure (e.g., shell) and viscoelastic and piezoelectric damping layers must be accurately considered. It is well known that modeling pure mechanical laminated shell structures is already a very complicated subject involving a lot of thinking concerning kinematic assumptions, displacement-strain-stress relationships, different variational principles, constitutive relations, consistency of the resultant governing equations, etc., with the extra complication of having the problem formulated in curvilinear coordinates. In addition, the viscoelastic and piezoelectric layers behaviors are also incorporated in the model increasing its complexity. That involves viscoelastic damping modeling issues in the one hand, utilized to describe the damping behavior of the viscoelastic counterpart of the structural system, and electro-mechanical coupling modeling assumptions for the piezoelectric layers counterpart in the other hand.

The finite element (FE) method is usually the preferable way of obtaining solutions for structures with more complicated geometries, boundary conditions and applied loads types. "Perfect" FEs of general shell structures without any numerical pathologies are still an issue to be solved by the scientific community and a challenge to deal with. However, models have been developed to simulate the structure and damping treatments and to assess their performance. The ultimate aim of this simulation is to define the optimal configuration (e.g., thickness, locations, number and type of sensors and actuators, control law, etc.) and put them in practice, making them work in order to solve some of the problems involving noise and vibration issues that our society nowadays has to deal with. Hence, one should also be familiarized with control theory aspects both for simulation or real-time implementation. These reasons attest the interesting multidisciplinarity and attracting challenge of modeling this type of coupled physical system, already reasonably studied over the last two decades, however with many aspects that still need to be solved. Further refinement of the models, fully coupling the physical ingredients, and coming up with some new approaches should be the main tendencies. There is still much work to be done and this research issue is far from being fully understood.

The derivation of shell theories has been one of the most prominent challenges in solid mechanics for many years. The idea is to develop appropriate models that can accurately simulate the effects of shear deformations and transverse normal strains in laminated shells with good trade-off between accuracy and complexity, which is a big mathematical difficulty. Physical 3-D shells are usually modeled recurring to approximated mathematical 2-D models. They are obtained by imposing some chosen kinematic and mechanical assumptions to the 3-D continuum, e.g., by explicitly assuming a through-the-thickness axiomatic displacement field definition and assuming a plane-stress state. When compared to 3-D solid FEs, 2-D shell FEs allow a significant reduction of the computational cost without losing much accuracy. However, to make matters worse, this sort of approximations lead to so-called locking effects (e.g., shear and membrane locking), which produces an overstiffening of the FE model which in turn produces erroneous results. Furthermore, for shell-type structures, with a more complex shear-membrane-bending coupling behavior, the locking effects are not yet fully understood yet, making these numerical pathologies difficult to remedy. Fully modeling the viscoelastic damping behavior is another important difficulty since their constitutive behavior is temperature and frequency dependent. Usually, for simplicity, only the frequency dependent material properties are considered and isothermal conditions are assumed.

When modeling this kind of structural system, various things have to be taken into account: the advances and alternative formulations of representative mathematical models of the physical behavior of shells with damping treatments; the developments (e.g., improved alternative configurations, control approaches, etc.) that the damping treatments suffered in the last decades; the developments and alternative approaches to viscoelastic damping modeling; considerations concerning the electro-mechanical coupling of piezoelectric layers; and the implementation and development of simple and reliable, locking-free, easily implemented FE solution methods. Over the years many authors have developed models for this type of problem. It's very difficult to comprehensively review their contributions because the multidisciplinarity of the subject makes the literature vast and the technical developments and improvements to appear dispersed in technical journals of different areas. However, the major disciplines, considered relevant for this work, are shortly reviewed here, namely: (1) elastic deformation theories, (2) piezoelectric materials and electro-mechanical coupling assumptions, (3) viscoelastic damping, (4) coupled piezo-visco-elastic FE formulations and (5) control strategies. This will allow to introduce the subject of this paper and to justify the options and assumptions taken for developing the model. Furthermore, it will allow to identify the aspects of the work that are new and significant in a more founded way.

In the development of structural mathematical models, different theories have been considered to axiomatically define the kinematics of laminated structural systems, where the planar dimensions are one to 
two orders of magnitude larger than their thickness (e.g., beams, plates or shells). Usually, these structural systems are formed by stacking layers of different isotropic or orthotropic composite materials with arbitrary fiber orientation or then, in this specific case, by arbitrary stacking sequences of active piezoelectric or passive viscoelastic damping layers.

These theories, following Kraus ${ }^{1}$ were originally developed for single layer "monocoque" thin structures made of traditional isotropic materials. Generally speaking, they can be grouped into two classes of alternate theories: one in which all of the Love's original assumptions ${ }^{2}$ are preserved, and other, following higher-order linear theories in which one or another of Love's assumptions are suspended. Many additional theories of thin and thick elastic shells have been proposed and the chronicle of these efforts are presented for example in Refs. 3 and 4.

Two different approaches are often used. The first one, the so-called Equivalent Single Layer (ESL) theories, where the number of independent generalized variables doesn't depend of the number of layers, are derived from 3-D elasticity by making suitable assumptions concerning the kinematics of deformation or the stress state through the thickness of the laminate, allowing the reduction of a 3-D problem to a 2-D one. The second one, the so-called Layerwise Theories (LWT), where the number of generalized variables depends on the number of physical (or nonphysical) layers, rely on the basis that the kinematic assumptions are established for each individual layer, which might be modeled (or not) as a 3-D solid. The problem is then reduced to a 2-D problem, however, retaining the 3-D intralaminar and interlaminar effects.

As reported by Yang et al., ${ }^{5}$ plate and shell structures made of laminated composite materials have often been modeled as an ESL using the classical laminate theory (CLT) (see for example the textbook of Reddy ${ }^{6}$ ), in which the out-of-plane stress components are ignored. The CLT is a direct extension of the well-known Kirchhoff-Love kinematic hypothesis, i.e., plane sections before deformation remain plane and normal to the mid-plane after deformation and that normals to the middle surface suffer no extension (Kirchhoff contribution) and others (cf. Ref.4), however applied to laminate composite structures. This theory is adequate when the ratio of the thickness to length (or other similar dimension) is small, the dynamic excitations are within the low-frequency range and the material anisotropy is not severe. The application of such theories to layered anisotropic composite shells could lead to $30 \%$ or more errors in deflections, stresses and frequencies. ${ }^{6}$ In order to overcome the deficiencies in the CLT, new refined laminate theories have been proposed relaxing some of the Love's postulates according to Koiter's recommendations, ${ }^{7}$ where it is stated that "... a refinement of Love's approximation theory is indeed meaningless, in general, unless the effects of transverse shear and normal stresses are taken into account at the same time." However, as stated by Carrera, ${ }^{8}$ for 2-D modelings of multilayered structures (such as laminated constructions, sandwich panels, layered structures used as thermal protection, intelligent structural systems embedding piezoelectric and/or viscoelastic layers) require amendments to Koiter's recommendation. Among these, the inclusion of continuity of displacements, zig-zag effects, and of transverse shear and normal stresses interlaminar continuity at the interface between two adjacent layers, are some of the amendments necessary. The role played by zig-zag effects and interlaminar continuity has been confirmed by many 3-D analysis of layered plates and shells. ${ }^{9-15}$ These amendments become more significant when complicating effects such as high in-plane and/or out-of-plane transverse anisotropy are present. Hence, as referred by Carrera, ${ }^{8}$ Koiter's recommendation concerning isotropic shells could be re-written for the case of multilayered shells as "... a refinement of ... unless the effects of interlaminar continuous transverse shear and normal stresses are taken into account at the same time." This enforces the need of also assuring interlaminar continuity of the out-of-plane stresses $\left(C_{z}^{0}\right.$ requirements $\left.{ }^{16}\right)$.

A refinement of the CLT, in which the transverse shear stresses are taken into account, was achieved with the extension to laminates of the so-called Reissner-Mindlin theory, or First-order transverse Shear Deformation Theory (FSDT). It provides improved global response estimates for deflections, vibration frequencies and buckling loads of moderately thick composites when compared to the CLT (see Ref. 6). Both approaches (CLT and FSDT) consider all layers as one anisotropic ESL and, as a consequence, they cannot model the warping effect of cross-sections. Furthermore, the assumption of a non-deformable normal results in incompatible shearing stresses between adjacent layers. The latter approach, because it assumes constant transverse shear stress, also requires the introduction of an arbitrary shear correction factor which depends on the lamination parameters for obtaining accurate results. Such a theory is adequate to predict only the gross behavior of laminates. Higher-Order Theories (HOTs), overcoming some of these limitations, were presented for example by Reddy ${ }^{17,18}$ for laminated plates and shells. However, because of the material mismatch at the intersection of the layers, the HOT also lead to transverse shear and normal stress mismatch at 
the intersection. In conclusion, ESL theories are found to be inadequate for detailed, accurate, local stress analysis of laminated structures.

If detailed response of individual layers is required, as is the case for piezoelectric layers, and if significant variations in displacements gradients between layers exist, as is the case of local phenomena usually in viscoelastic layers, LWT (discrete layer) become more suitable to model the intralaminar and interlaminar effects and the warping of the cross section. The LWT corresponds to the implementation of CLT, FSDT or HOT at a layer level. That is, each layer is seen as an individual plate or shell and compatibility (continuity) of displacement (and eventually out-of-plane stress) components with correspondence to each interface is imposed as a constraint. As can be seen in Refs. 19 and 20, high-order displacement-based or mixed LWT have been successfully used to accurately model the behavior of laminates taking into account the interlaminar and intralaminar effects.

Another alternative, with a reduced computational effort, in the framework of ESL theories, is the use of the so-called Zig-Zag Theories (ZZTs), which have their origins and most significant contributions coming from the Russian school. Refined ZZT have therefore been motivated to fulfill a priori (in a complete or partial form) the $C_{z}^{0}$ requirements. The fundamental ideas in developing ZZT consists to assume a certain displacement and/or stress model in each layer and then to use compatibility and equilibrium conditions at the interface to reduce the number of the unknown variables and keep the number of variables independent of the number of layers.

As stated by Carrera, ${ }^{21}$ the first contribution to the ZZT was supposedly given by Lekhnitskii in the 1930s (see the brief treatment concerning a layered beam in the English translation of his book [22, Section 18]). Apart from the method proposed by Lekhnitskii, which was almost ignored, two other independent contributions, which received much more attention from the scientific community, have been proposed in the literature in the second half of last century. The first of these was originally given by Ambartsumian in the $1950 \mathrm{~s}$, motivated by the attempt to refine the CLT to include partially or completely the $C_{z}^{0}$ requirements, and was applied to anisotropic single and multilayer plates and shells (see his textbooks ${ }^{23,24}$ ). Several variations have been presented which consisted in direct or particular applications of the original Ambartsumian's idea. Whitney [25, Chapter 7] introduced the theory in the Western community and applied it to nonsymmetrical plates whereas the extension to multilayer shells and dynamic problems was made by Rath and Das. ${ }^{26}$ However, several unuseful works concerning particular applications of Ambartsumian's original theory were developed presenting progressive refinements towards the original idea. It was only in the 1990s that the original theory was re-obtained as can be found, among others, in the works of Cho and Parmerter, ${ }^{27}$ Beakou and Touratier ${ }^{28}$ and Soldatos and Timarci. ${ }^{29}$ Regarding the second independent contribution, it was given by Reissner in 1980s, ${ }^{30}$ who proposed a mixed variational theorem that allows both displacements and stress assumptions to be made. Significant contributions to the theory proposed by Reissner were made by Murakami $^{31}$ that introduced a zig-zag form of displacement field and Carrera ${ }^{32}$ that presented a systematic generalized manner of using the Reissner mixed variational principle to develop FE applications of ESL theories and LWT of plates and shells. For further details, an historical review of ZZT was performed by Carrera. ${ }^{21,33}$ A discussion on the theories and FE for multilayered structures, with numerical assessment and a benchmarks for plate and shell structures can be found in the literature. ${ }^{33-39}$

When modeling piezoelectric sensors and actuators, different electrical assumptions can be taken into account in the theoretical model when considering the electro-mechanical (or piezo-elastic) coupling. These assumptions regard mainly the use (or not) of electric degrees of freedom (DoFs) and the approximations of the in-plane and out-of-plane components of the electric displacement vector and/or through-the-thickness variation of the electric potential. Therefore, they might lead to decoupled, partial and fully coupled electromechanical theories, which in turn can lead to different modifications of the structure's stiffness and different approximations of the physics of the system. Those electro-mechanical coupling theories can be considered by the use of effective stiffness parameters (ESP), defined according to the electric boundary condition considered, as shown in Refs. 40-42 for a smart piezoelectric beam and plate. This ESP formulation, however, can also be extended to other types of structures such as shells. Interesting textbooks concerning the piezoelectric modeling of beams, plates and shells can be found in Refs. 43-45.

In the last decade the technology associated with a more refined design and adequate implementation of viscoelastic-based damping treatments has achieved a relative maturity and is frequently applied in practice by the scientific community and its industrial partners. Active Constrained Layer Damping (ACLD) treatments have revealed from the early 1990s to be an effective means of vibration suppression. ${ }^{46}$ In an attempt to improve performance, different configurations of the active (piezoelectric) and passive (viscoelastic) con- 
straining layers have been used since then. These treatments are called arbitrary ACLD treatments or hybrid active-passive damping treatments. The ACLD treatments combine the high passive capacity of viscoelastic materials to dissipate vibratory energy at high frequencies with the active capacity of piezoelectric materials at low frequencies. Therefore, in the same damping treatment, a broader band control is achieved benefiting from the advantages of both passive (simplicity, stability, fail-safe, low-cost) and active (adaptability, highperformance) systems. In order to adequately design these viscoelastic damping treatments the viscoelastic material properties need to be accurately measured and considered for the underlying mathematical models. The temperature and frequency dependent material properties of the viscoelastic materials complicate the mathematical model and make the solution of the problem more difficult to obtain. Usually, isothermal conditions are assumed and only the frequency dependent constitutive behavior is taken into account. The simplest way of modeling these materials is achieved by a Complex Modulus Approach (CMA) where the material properties are defined for each discrete frequency value. ${ }^{47,48}$ The CMA is also associated with the so-called Modal Strain Energy (MSE) method ${ }^{49}$ where the loss factors of each individual mode are determined from the ratios between the dissipated modal strain energy of the viscoelastic counterpart and the storage modal strain energy of the global structural system. The MSE method is known to lead to poor viscoelastic damping estimation of highly damped structural systems. Furthermore, the CMA is a frequency domain method that is limited to steady state vibrations and single-frequency harmonic excitations. Thus, to account for the frequency dependent material properties, iterative versions of the MSE have been used successfully for moderate damping values. ${ }^{50}$ Time domain models, relying on internal variables (see Johnson $^{51}$ ), such as the Golla-Hughes-McTavish $(\mathrm{GHM})^{52,53}$ and Anelastic Displacement Fields (ADF), ${ }^{54,55}$ or others, ${ }^{56,57}$ utilizing additional dissipation variables, have been successfully utilized and yield good damping estimates. Alternatively, the use of Fractional Calculus (FC) ${ }^{58,59}$ models, based upon the use of fractional derivatives, has the drawback of generating a "non-standard" FE formulation, with a more complex characteristic solution procedure, but yielding also good damping estimates. Other relevant contribution for the viscoelastic damping modeling is given by Adhikari's work in Ref. 60 and his references therein. Regarding the temperature effects, studies taking into account the temperature dependence of the properties and the self-heating of viscoelastic materials have been performed by Lesieutre and his co-workers in Refs. 61 and 62 which extended the ADF model for these cases leading, however, to nonlinear differential equations. The effects of the operating temperature on hybrid damping treatments performance and viscoelastic damping efficiency were analyzed, for example, in Refs. 63-67.

Time domain models represent better alternatives to CMA-based models allowing the reduction of the computational burden and the study of the transient response in a more straightforward manner, even for highly damped structural systems. Among the time domain models, internal variables models are more interesting from the computational point of view and easiness of implementation into FE codes. Thus, the GHM and ADF models are alternative methods, used to model the damping behavior of viscoelastic materials in FE analysis, which yield a standard FE formulation (however with the addition of some "non-physical" dissipative variables). In order to use them, one needs the GHM and ADF characteristic parameters which allow characterizing the complex (frequency dependent) constitutive behavior of the viscoelastic material being used. To do that, experimental procedures to measure the isotropic constitutive behavior (usually the shear modulus) and numerical identification procedures of the measured data need to be developed (see Vasques et al. $\left.{ }^{68}\right)$.

When designing hybrid active-passive treatments (see the review articles in Ref. 69 and 70) it is important to know the configuration of the structure and treatment that gives optimal damping. For simulation the designer needs a model of the system in order to define the optimal locations, thicknesses, configurations, control law, etc. Thus, there are numerous options at the design stage. Modeling this kind of structural systems usually requires a coupled piezo-visco-elastic structural FE model, comprising the piezoelectric, viscoelastic and elastic layers constitutive behaviors. Surveys on the advances in FE modeling of piezoelectric adaptive structures with or without hybrid active-passive damping treatments are presented by Benjeddou. ${ }^{71,72} \mathrm{~A}$ review and assessments of hybrid treatments on beams is also performed by Trindade. ${ }^{73}$

In the last decades the advances in digital signal processing and sensors and actuators technology have prompted interest in active control and a considerable effort has been put in the development and implementation of Active Noise Control (ANC) and Active Vibration Control (AVC) theories (see related textbooks $\left.{ }^{74-81}\right)$. These might be divided into two fundamental classes, namely, feedback and feedforward control algorithms. The former control strategy has been shown to be most suitable in applications where the structure is under impulsive or stochastic unknown disturbances and the latter to the case where deter- 
ministic or correlated information about the disturbance is known. Variations of the two general methods exist, each with advantages, disadvantages and limitations. A review paper concerning active structural vibration control strategies is presented by Alkhatib and Golnaraghi. ${ }^{82}$

In the open literature several studies concerning different control of vibration strategies (e.g., feedforward wave suppression, proportional and velocity feedback, optimal control) with different types of actuation (e.g., point forces, pair of moments, piezoelectric patch actuation) in different structural systems with piezoelectric actuators or hybrid active-passive treatments can be found in Refs. 83-86. Comparisons of different classical and/or optimal feedback control strategies were performed, for example, by Gandhi and Munsky ${ }^{87}$ and Vasques and Rodrigues. ${ }^{88}$ While feedback control has been vastly applied in the vibration control of structures with arbitrary ACLD (hybrid active-passive) damping treatments, only a few works utilizing feedforward theory can be found in the open literature. ${ }^{89-91}$ The most typical application of feedforward control presented in textbooks concerns the noise attenuation in ducts and applications concerning vibration reduction are mainly devoted to Active Structural Acoustic Control (ASAC). Furthermore, hybrid (combined feedback/feedforward) control strategies were successfully used in the past for noise and vibration suppression (see for example Refs. 92-95). However, only one study concerning hybrid control with ACLD treatments applied to a beam was performed by Vasques and Rodrigues. ${ }^{96}$

This paper concerns the mathematical modeling and FE solution of general anisotropic shells with hybrid active-passive damping treatments. A fully-coupled piezo-visco-elastic mathematical model of the shell (host structure) and segmented arbitrarily stacked layers of damping treatments is considered in the framework of a discrete layer approach. Thus, the weak form of the governing equations is derived for a single generic layer of the multilayer shell using Hamilton's principle and a mixed (displacement/stresses) definition of the displacement field.

First, a fully refined deformation theory of the generic layer, based on postulated out-of-plane shear stress components and the in-plane stresses obtained with a Reissner-Mindlin type shell theory, is outlined. A semiinverse iterative procedure is used to derive the layer mixed non-linear displacement field, in terms of a blend of the generalized displacements of the Love-Kirchhoff and Reissner-Mindlin theories and stress components at the generic layer interfaces, without any simplifying assumptions regarding the thinness of the shell being considered. The electrical potential is defined assuming negligible in-plane electric displacement field components, and a constant transverse electric displacement. Regarding the electro-mechanical coupling, a fully coupled theory is considered where the direct piezoelectric effect is adequately taken into account and the converse piezoelectric effect is considered by the action of a prescribed electric potential difference in the generic piezoelectric layer surface electrodes.

Then, the weak forms of a partially refined theory, where only the zero-order term of the non-linear fully refined transverse displacement is retained, are derived for an orthotropic doubly-curved piezo-elastic generic layer. Based on the weak forms a FE solution is developed for the generic shell layer. The degrees of freedom (DoFs) of the resultant four-noded generic piezo-elastic single layer FE are then "regenerated" into an equivalent eight-node 3-D formulation in order to allow through-the-thickness assemblage of displacements and stresses and the generation of a partially refined multilayer piezo-elastic FE. A dynamic condensation technique is employed to eliminate the stress DoFs and to cast the problem in an equivalent displacementbased FE model form.

Last but not the least, the viscoelastic damping behavior is considered by means of a Laplace transformed ADF model implemented at the global FE model level and a partially refined piezo-visco-elastic multilayer FE model of orthotropic doubly-curved shells is presented. The active control of vibration is shortly discussed and a set of indices to quantify the damping performance and the individual contributions of the different mechanisms are proposed, allowing the phenomenological study and the design of the hybrid damping treatments in a more straightforward manner.

Regarding the deformation theory developed in this work, it is inspired in Ambartsumian's contributions for the deformation theory of single layer anisotropic plates and shells. ${ }^{23,24,97}$ Ambartsumian basically used a semi-inverse method to develop refined shear deformation theories. They are based on assuming a refined distribution of the transverse shear stresses and in the use of the the equilibrium and constitutive equations to derive expressions for the in-plane displacements, which in turn become non-linear in the thickness coordinate. Improvements including the effect of transverse normal strain were also considered for plates and shells. In some of these refined theories transverse shear stress distributions are assumed to follow a parabolic law and to satisfy zero shear stress conditions at the top and bottom surfaces of the plate or shell. However, if out-of-plane stresses continuity is required, a mixed formulation should consider also the stresses on the 
interfaces of the single layer, which, for the sake of simplicity, were, in general, assumed to be nil in the single layer theories developed by Ambartsumian. That puts some limitations in the generalization of the theory to multilayer structures since neither the displacement nor the normal stresses are available in the interfaces and interlayer continuity can not be imposed. Furthermore, his multilayer approach doesn't allow to consider segmented layers, which is something required in the study of segmented hybrid damping treatments. When that is the case, individual refined theories must be considered for each individual discrete-layer.

When compared with Ambartsumian's first and second improved theories of anisotropic shells (see Ref. 24), the proposed fully and partially refined theories, similar to Ambartsumian's first and second improved theories, respectively, assume as a first approximation of the in-plane stresses the ones obtained with the FSDT instead of the CLT. Additionally, all the surface shear stresses are retained in the formulation since they will be used afterward with the "regenerated" 3-D element to generalize the theory to segmented multilayered shells. Furthermore, the theory is extended to coupled piezo-visco-elastic multilayered shells and a FE solution is developed. Thus, however strongly inspired in Ambartsumian's work, the underlying deformation theory of the present work has some important differences and represents a further step towards the increasing refinement of this type of multiphysics problem. It is worthy to mention that it would be very complicated and cumbersome to fulfill a priori all the $C_{z}^{0}$ requirements for a multilayer anisotropic shell. Instead, a discrete layer approach is used, which allows interlayer displacement and out-of-plane stresses continuity to be imposed a posteriori in a more straightforward manner, by means of a through-the-thickness assemblage of the "regenerated" single layer FE.

Regarding the variational approach used to get the governing equations, similar analytical works using "partial" mixed theories where the displacement field is defined in terms of generalized displacements and generalized surface and transverse stresses can be found for beams and plates in the works of Rao et al. ${ }^{98-100}$ In contrast to other "fully" mixed methods, as the ZZT based on Reissner's contribution, where mixed variational principles are used, Hamilton's principle has also been employed to derive the governing equations (i.e., no mixed-enhanced variational principles are considered). The present work extends a similar concept to multilayered shells and a FE solution is developed. Concerning piezo-visco-elastic FEs, a similar work about a three-layered coupled piezo-visco-elastic plate FE was developed by Chattopadhyay et al., ${ }^{101}$ where a HOT was used for the definition of the displacement field of each individual layer and the displacement and shear stress continuity were assured. However, the formulation is limited to the study of active constrained layer damping (ACLD) treatments on plates and doesn't allow the study of arbitrary damping treatments (multilayer structures). As far as the "regeneration" concept is concerned, the concept has been employed also by Cho and Averill ${ }^{102}$ in the the framework of the ZZTs, where a plate FE, avoiding the shortcomings of requiring $C^{1}$ continuity of the transverse displacement, has been developed using a first-order zig-zag sublaminate theory for laminated composite and sandwich panels. However, the formulation is based on the decomposition of the whole structure in sublaminates, a linear piecewise function is assumed for the displacement of each sublaminate and the interlaminar displacement and normal shear stress continuity is imposed between the layers of the same sublaminate but not between the sublaminates. In comparison, the present work uses a similar "regeneration" concept to shell-type structures with a more refined deformation theory being employed which allows displacement and interlayer continuity between all layers to be imposed.

To sum up, this work will present some new developments to the study of shells with hybrid damping treatments. Only a few papers concerning hybrid active-passive damping treatments in shells are found in the open literature. They were mainly devoted to simple shell geometries and a few to arbitrary shells. Therefore further studies on shells are necessary. Here one intends to develop an accurate mechanical structural model of shells, considering also an accurate model of the electro-mechanical coupling and an effective time domain damping model of the viscoelastic layers. Furthermore, works in the open literature are usually devoted to three-layered sandwich shells and usually concern only modeling aspects. It's also of great importance the phenomenological study and quantification of the damping mechanisms for different configurations of the treatments in shells, which are not fully understood yet. These are the major novelties of this work, which comprises accurate modeling issues on the one hand and phenomenological study of the treatments for hybrid, active or passive vibration control on the other hand. 


\section{Fully Refined Mathematical Model of General Shells}

\section{A. Physical Problem Description}

The physical problem to be mathematically modeled here concerns a general anisotropic shell to which damping treatments of piezoelectric and viscoelastic materials are attached on both top and bottom surfaces of the host structure (Fig. 1). All the damping layers and the host structure have constant thickness. The damping treatments are required to be able to be discontinuous (or segmented) and arbitrary stacking sequences of hybrid damping treatments comprising piezoelectric sensing or actuating, viscoelastic or elastic layers are considered. Isotropic viscoelastic materials are used and the piezoelectric materials are general orthotropic materials of the class $m m 2 .{ }^{103,104}$ The system is supposed to operate under moderate changing thermal environments, well bellow the Curie temperature of the piezoelectric material.

Thus, the physical problem involves visco-elastic (or mechanical) and piezo-elastic (or electro-mechanical) interactions mainly devoted to increase the energy dissipation of the structural system.

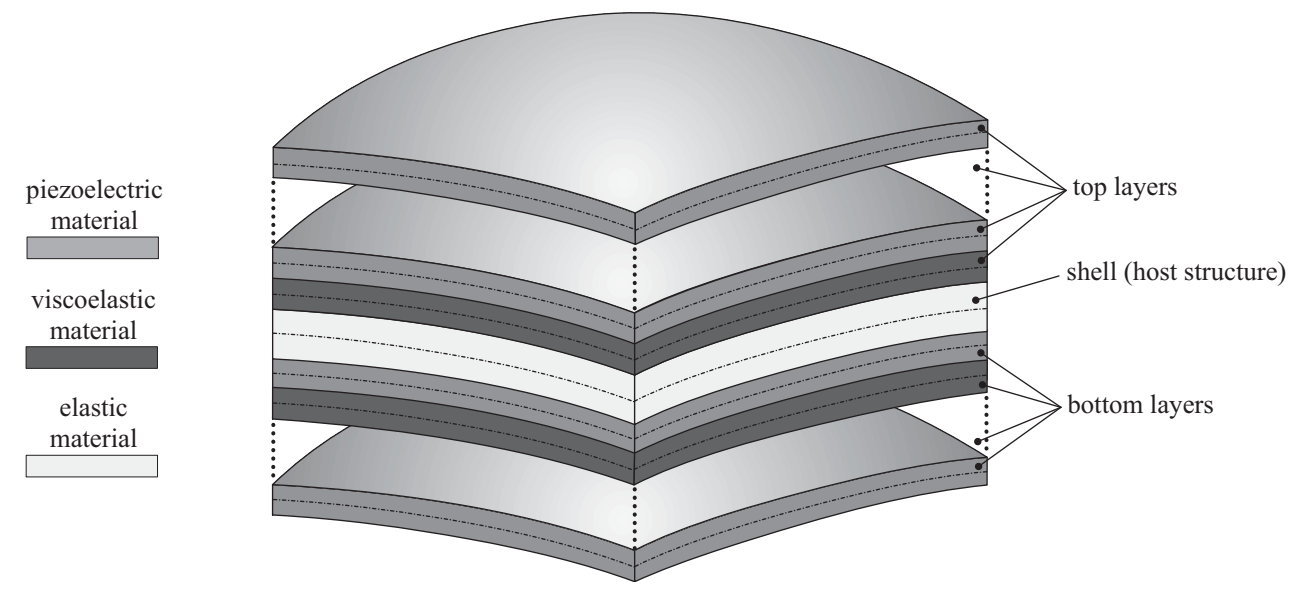

Figure 1. Multilayer shell with arbitrary hybrid damping treatments.

\section{B. Shell Differential Geometry}

A theory is established by treating the shell with hybrid damping treatments as a multilayer shell of arbitrary materials. Then, a generic layer (elastic, viscoelastic or piezoelectric) is taken from the global laminate, and the week form of the governing equations is derived for the individual layer. Interlaminar (or interlayer) continuity conditions of displacements and stresses (surface tractions) and homogeneous (or not) traction boundary conditions at inner and outer boundary surfaces of the global laminate (or damped shell) are imposed later, at the multilayer FE level, by assembling all the individual layers contributions.

Consider a generic piezo-visco-elastic layer (Fig. 2) extracted from the multilayer shell in Fig. 1. The general orthotropic (anisotropic) layer has a constant thickness of $2 h$ and a plane of elastic symmetry parallel to the middle surface $\Omega_{0}$. The latter surface is used as a reference surface referred to the curvilinear orthogonal coordinates $\alpha$ and $\beta$, which coincide with the lines of principal curvature of the middle surface. Let $z$ denote the distance comprised in the interval $[-h, h]$ and measured along the normal of a point $(\alpha, \beta)$ of the middle surface $\Omega_{0}$ and a point $(\alpha, \beta, z)$ of the shell layer and $\Omega$ a surface at a distance $z$ and parallel to $\Omega_{0}$. The square of an arbitrary differential element of arc length $\mathrm{d} s$, the infinitesimal area of a rectangle in $\Omega$ and an infinitesimal volume $\mathrm{d} V$ are given by

$$
d s^{2}=H_{\alpha}{ }^{2} d \alpha^{2}+H_{\beta}{ }^{2} d \beta^{2}+H_{z}{ }^{2} d z^{2}, \quad d \Omega=H_{\alpha} H_{\beta} d \alpha d \beta, \quad d V=H_{\alpha} H_{\beta} H_{z} d \alpha d \beta d z,
$$

where $H_{\alpha}=H_{\alpha}(\alpha, \beta, z), H_{\beta}=H_{\beta}(\alpha, \beta, z)$ and $H_{z}$ are the so-called Lamé parameters given by

$$
H_{\alpha}=A_{\alpha}\left(1+\frac{z}{R_{\alpha}}\right), \quad H_{\beta}=A_{\beta}\left(1+\frac{z}{R_{\beta}}\right), \quad H_{z}=1 .
$$


$A_{\alpha}=A_{\alpha}(\alpha, \beta)$ and $A_{\beta}=A_{\beta}(\alpha, \beta)$ are the square root of the coefficients of the first fundamental form and $R_{\alpha}=R_{\alpha}(\alpha, \beta)$ and $R_{\beta}=R_{\beta}(\alpha, \beta)$ the principal radii of curvature of the middle surface $\Omega_{0}$ (see for example Kraus $^{1}$ for further details).

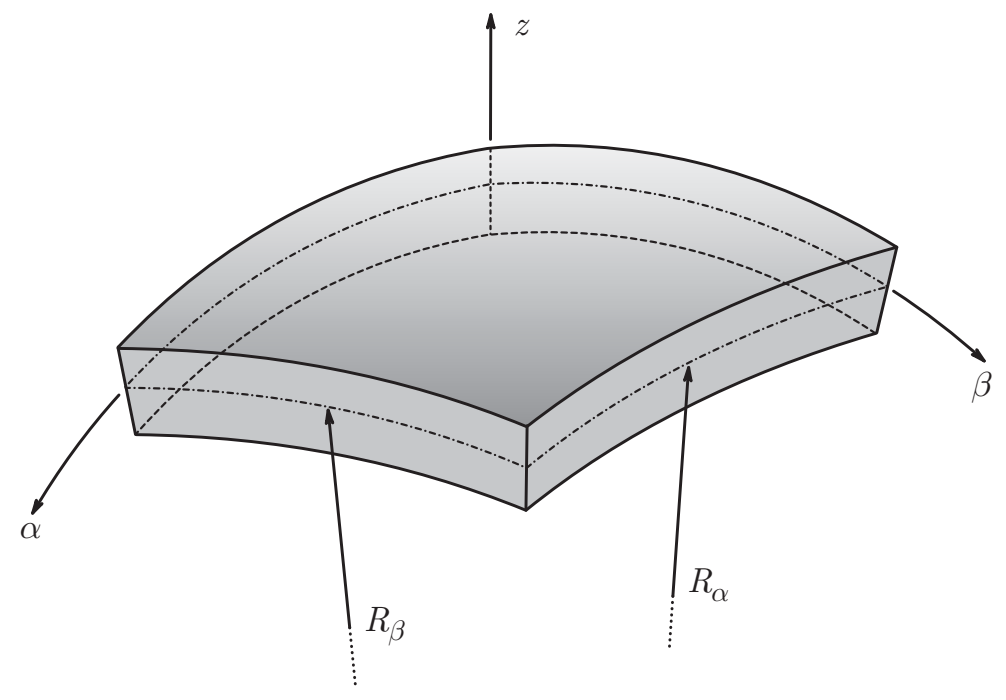

Figure 2. Generic piezo-visco-elastic shell layer.

\section{General Assumptions and Relationships}

Different assumptions are made regarding the mechanical, electrical and electro-mechanical behavior. By now, one will be only concerned with the mechanical assumptions which are as follows:

(a) Strains and displacements are sufficiently small so that the quantities of the second- and higher-order magnitude in the strain-displacement relationships may be neglected in comparison with the first-order terms (infinitesimal strains and linear elasticity);

(b) The shear stresses $\sigma_{z \alpha}(\alpha, \beta, z)$ and $\sigma_{\beta z}(\alpha, \beta, z)$, or the corresponding strains $\varepsilon_{z \alpha}(\alpha, \beta, z)$ and $\varepsilon_{\beta z}(\alpha, \beta, z)$, vary in the shell layer thickness according to a specified law, defined by a "correction" even function $f(z)$, symmetric relative to the middle surface (e.g., parabolic, trigonometric), and the shear angle rotations $\psi_{\alpha}(\alpha, \beta)$ and $\psi_{\beta}(\alpha, \beta)$ of a normal to the reference mid-surface obtained from the FSDT; additionally non-homogeneous conditions are assumed at the top and bottom surfaces;

(c) The normal stresses $\sigma_{z z}(\alpha, \beta, z)$ at areas parallel to the middle surface are not negligible and are obtained through the out-of-plane equilibrium equation in orthogonal curvilinear coordinates, and non-homogeneous conditions are assumed at the top and bottom surfaces;

(d) The strain $\varepsilon_{z z}(\alpha, \beta, z)$ is determined assuming as first approximations of the in-plane stresses the ones obtained with the FSDT for shells defined in Appendix B, $\sigma_{\alpha \alpha}^{*}(\alpha, \beta, z), \sigma_{\beta \beta}^{*}(\alpha, \beta, z)$ and $\sigma_{\alpha \beta}^{*}(\alpha, \beta, z)$, without any simplification regarding the thinness of the shell being made (i.e., the terms $z / R_{\alpha}(\alpha, \beta)$ and $z / R_{\beta}(\alpha, \beta)$ are fully retained);

According to assumption (b) the out-of-plane shear stress components are postulated as

$$
\begin{aligned}
& \sigma_{z \alpha}(\alpha, \beta, z)=\frac{1}{H_{\alpha}}\left[\bar{\tau}_{z \alpha}(\alpha, \beta)+\frac{z}{2 h} \tilde{\tau}_{z \alpha}(\alpha, \beta)+f(z) \psi_{\alpha}(\alpha, \beta)\right], \\
& \sigma_{\beta z}(\alpha, \beta, z)=\frac{1}{H_{\beta}}\left[\bar{\tau}_{\beta z}(\alpha, \beta)+\frac{z}{2 h} \tilde{\tau}_{\beta z}(\alpha, \beta)+f(z) \psi_{\beta}(\alpha, \beta)\right],
\end{aligned}
$$


where $\psi_{\alpha}=\psi_{\alpha}(\alpha, \beta)$ and $\psi_{\beta}=\psi_{\beta}(\alpha, \beta)$ are the shear angles obtained with the FSDT (defined in Appendix B) and the bar and tilde above $\tau_{z \alpha}$ and $\tau_{\beta z}$ are used to denote mean imposed surface shear tractions, given by

$$
\bar{\tau}_{z \alpha}(\alpha, \beta)=\frac{1}{2}\left[\sigma_{z \alpha}^{t}(\alpha, \beta)-\sigma_{z \alpha}^{b}(\alpha, \beta)\right], \quad \bar{\tau}_{\beta z}(\alpha, \beta)=\frac{1}{2}\left[\sigma_{\beta z}^{t}(\alpha, \beta)-\sigma_{\beta z}^{b}(\alpha, \beta)\right],
$$

and relative ones, given by

$$
\tilde{\tau}_{z \alpha}(\alpha, \beta)=\sigma_{z \alpha}^{t}(\alpha, \beta)+\sigma_{z \alpha}^{b}(\alpha, \beta), \quad \tilde{\tau}_{\beta z}(\alpha, \beta)=\sigma_{\beta z}^{t}(\alpha, \beta)+\sigma_{\beta z}^{b}(\alpha, \beta),
$$

where $(\cdot)^{t}$ and $(\cdot)^{b}$ denote prescribed shear tractions at the top and bottom surfaces (i.e., at $z= \pm h$ ), respectively, of the shell layer (see Fig. 3).

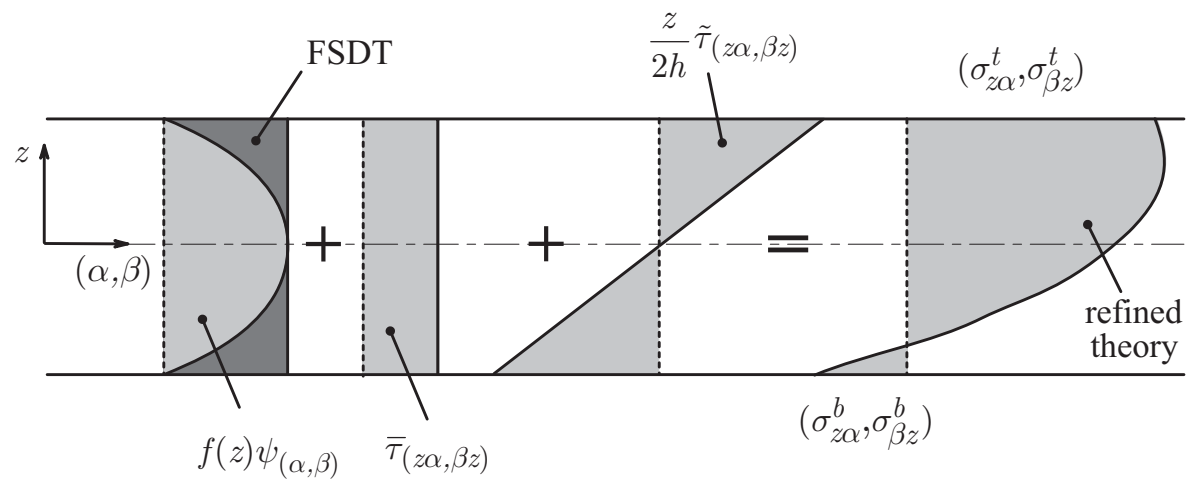

Figure 3. Postulated shear strains distributions of the refined theory.

By substituting the values of $\sigma_{z \alpha}(\alpha, \beta, z)$ and $\sigma_{\beta z}(\alpha, \beta, z)$ in Eqs. (3) into the static transverse equilibrium equation in orthogonal curvilinear coordinates in the third of Eqs. (A2) of the Appendix, in the absence of body forces, taking into account the definitions in (4) and (5) and integrating with respect to $z$, yields the following relation for the normal stress component $\sigma_{z z}(\alpha, \beta, z)$ defined in terms of coefficients of powers of $z$ (since they are not important here, for the sake of simplicity, the necessary definitions will be given latter),

$$
\sigma_{z z}(\alpha, \beta, z)=\sigma_{z z}^{(0)}(\alpha, \beta)+\sigma_{z z}^{(1)}(\alpha, \beta, z)+\sigma_{z z}^{(2)}(\alpha, \beta, z)+\sigma_{z z}^{(f)}(\alpha, \beta, z)
$$

where $\sigma_{z z}^{(0)}(\alpha, \beta)$ is an integration function independent of $z$ that is determined from the boundary conditions on the top and bottom surfaces of the shell layer, $\sigma_{z z}^{t}(\alpha, \beta)$ and $\sigma_{z z}^{b}(\alpha, \beta)$, respectively. Regarding the dependencies of the different order terms, the first-order one, $\sigma_{z z}^{(1)}(\alpha, \beta, z)$, depends of the mean shear tractions $\bar{\tau}_{z \alpha}(\alpha, \beta)$ and $\bar{\tau}_{\beta z}(\alpha, \beta)$, the second-order term, $\sigma_{z z}^{(2)}(\alpha, \beta, z)$, depends of the relative shear tractions $\tilde{\tau}_{z \alpha}(\alpha, \beta)$ and $\tilde{\tau}_{\beta z}(\alpha, \beta)$, and the term depending on $f(z), \sigma_{z z}^{(f)}(\alpha, \beta, z)$, is related with the shear angles $\psi_{\alpha}(\alpha, \beta)$ and $\psi_{\beta}(\alpha, \beta)$.

By satisfying the prescribed stress conditions $\sigma_{z z}^{t}(\alpha, \beta)$ and $\sigma_{z z}^{b}(\alpha, \beta)$ at the top and bottom surfaces, after some algebra, the function dependent of $(\alpha, \beta)$ (integration constant in $z$ ) that results from the integration is given as

$$
\sigma_{z z}^{(0)}(\alpha, \beta)=\bar{\tau}_{z z}(\alpha, \beta)-h^{2} \sigma_{z z}^{(2)}(\alpha, \beta)-\frac{[F(h)+F(-h)]}{2 F(z)} \sigma_{z z}^{(f)}(\alpha, \beta, z)=\bar{\tau}_{z z}(\alpha, \beta)-h^{2} \sigma_{z z}^{(2)}(\alpha, \beta),
$$

where

$$
F(z)=\int f(z) d z .
$$

Eq. (7) is simplified since $\sigma_{z z}^{(0)}(\alpha, \beta)$ can't depend on $z$, which is confirmed since $F(z)$ is and odd function, i.e., $F(h)=-F(-h)$. Additionally, an extra equation is obtained by imposing the top and bottom conditions, which defines the term $\sigma_{z z}^{(f)}(\alpha, \beta, z)$ as

$$
\sigma_{z z}^{(f)}(\alpha, \beta, z)=\frac{F(z)}{F(h)-F(-h)}\left[\tilde{\tau}_{z z}(\alpha, \beta)-2 h \sigma_{z z}^{(1)}(\alpha, \beta, z)\right],
$$


where similarly to Eqs. (4) and (5), the bar and tilde have been used to denote mean and relative prescribed transverse stresses,

$$
\bar{\tau}_{z z}(\alpha, \beta)=\frac{1}{2}\left[\sigma_{z z}^{t}(\alpha, \beta)-\sigma_{z z}^{b}(\alpha, \beta)\right], \quad \tilde{\tau}_{z z}(\alpha, \beta)=\sigma_{z z}^{t}(\alpha, \beta)+\sigma_{z z}^{b}(\alpha, \beta) .
$$

After some algebra, the remaining undefined terms of Eq. (6) are given by

$$
\begin{gathered}
\sigma_{z z}^{(1)}(\alpha, \beta, z)=\frac{z}{H_{\alpha} H_{\beta}}\left[H_{\beta} \frac{A_{\alpha}}{R_{\alpha}} \sigma_{\alpha \alpha}^{*(0)}(\alpha, \beta, z)+H_{\alpha} \frac{A_{\beta}}{R_{\beta}} \sigma_{\beta \beta}^{*(0)}(\alpha, \beta, z)-\frac{\partial \bar{\tau}_{z \alpha}(\alpha, \beta)}{\partial \alpha}-\frac{\partial \bar{\tau}_{\beta z}(\alpha, \beta)}{\partial \beta}\right], \\
\sigma_{z z}^{(2)}(\alpha, \beta, z)=\frac{z^{2}}{H_{\alpha} H_{\beta}}\left[H_{\beta} \frac{A_{\alpha}}{2 R_{\alpha}} \sigma_{\alpha \alpha}^{*(1)}(\alpha, \beta, z)+H_{\alpha} \frac{A_{\beta}}{2 R_{\beta}} \sigma_{\beta \beta}^{*(1)}(\alpha, \beta, z)-\frac{1}{4 h} \frac{\partial \tilde{\tau}_{z \alpha}(\alpha, \beta)}{\partial \alpha}-\frac{1}{4 h} \frac{\partial \tilde{\tau}_{\beta z}(\alpha, \beta)}{\partial \beta}\right] .
\end{gathered}
$$

At this point, it's worthy to mention that in order to keep the formulation of the transverse stress $\sigma_{z z}(\alpha, \beta, z)$ in Eq. (6) general, its last term depends of the integral of the shear "correction" function, $F(z)$. Depending of the type and/or order in $z$ of the shear function $f(z)$, (e.g., quadratic polynomial, trigonometric function), different expansions in $z$ can be obtained.

Considering the strain-stress constitutive behavior of the out-of-plane shear strains $\varepsilon_{z \alpha}$ and $\varepsilon_{\beta z}$ expressed in Eq. (C14) in Appendix, the shear strains are expressed as

$$
\begin{aligned}
& \varepsilon_{z \alpha}(\alpha, \beta, z)=\frac{1}{H_{\alpha}}\left[\bar{\Sigma}_{z \alpha}(\alpha, \beta)+\frac{z}{2 h} \tilde{\Sigma}_{z \alpha}(\alpha, \beta)+f(z) \Psi_{\alpha}(\alpha, \beta)\right], \\
& \varepsilon_{\beta z}(\alpha, \beta, z)=\frac{1}{H_{\beta}}\left[\bar{\Sigma}_{\beta z}(\alpha, \beta)+\frac{z}{2 h} \tilde{\Sigma}_{\beta z}(\alpha, \beta)+f(z) \Psi_{\beta}(\alpha, \beta)\right],
\end{aligned}
$$

where the following notations regarding the mean surface tractions,

$$
\bar{\Sigma}_{z \alpha}(\alpha, \beta)=\bar{s}_{45}^{\star} \bar{\tau}_{\beta z}(\alpha, \beta)+\bar{s}_{55}^{\star} \bar{\tau}_{z \alpha}(\alpha, \beta), \quad \bar{\Sigma}_{\beta z}(\alpha, \beta)=\bar{s}_{44}^{\star} \bar{\tau}_{\beta z}(\alpha, \beta)+\bar{s}_{45}^{\star} \bar{\tau}_{z \alpha}(\alpha, \beta),
$$

relative surface tractions,

$$
\tilde{\Sigma}_{z \alpha}(\alpha, \beta)=\bar{s}_{45}^{\star} \tilde{\tau}_{\beta z}(\alpha, \beta)+\bar{s}_{55}^{\star} \tilde{\tau}_{z \alpha}(\alpha, \beta), \quad \tilde{\Sigma}_{\beta z}(\alpha, \beta)=\bar{s}_{44}^{\star} \tilde{\tau}_{\beta z}(\alpha, \beta)+\bar{s}_{45}^{\star} \tilde{\tau}_{z \alpha}(\alpha, \beta),
$$

and shear angle rotations,

$$
\Psi_{\alpha}(\alpha, \beta)=\bar{s}_{45}^{\star} \psi_{\beta}(\alpha, \beta)+\bar{s}_{55}^{\star} \psi_{\alpha}(\alpha, \beta), \quad \Psi_{\beta}(\alpha, \beta)=\bar{s}_{44}^{\star} \psi_{\beta}(\alpha, \beta)+\bar{s}_{45}^{\star} \psi_{\alpha}(\alpha, \beta),
$$

are used in terms of effective shear compliance coefficients (see Appendix C). Alternatively, for simplicity, the shear strains in Eqs. (13) can be expressed in terms of coefficients of increasing powers and functions of $z$ as

$$
\begin{aligned}
& \varepsilon_{z \alpha}(\alpha, \beta, z)=\frac{1}{H_{\alpha}}\left[\varepsilon_{z \alpha}^{(0)}(\alpha, \beta)+z \varepsilon_{z \alpha}^{(1)}(\alpha, \beta)+\varepsilon_{z \alpha}^{(f)}(\alpha, \beta, z)\right], \\
& \varepsilon_{\beta z}(\alpha, \beta, z)=\frac{1}{H_{\beta}}\left[\varepsilon_{\beta z}^{(0)}(\alpha, \beta)+z \varepsilon_{\beta z}^{(1)}(\alpha, \beta)+\varepsilon_{\beta z}^{(f)}(\alpha, \beta, z)\right],
\end{aligned}
$$

where the definitions of the terms in the previous equations are obvious from the analysis of Eqs. (13).

In a similar way, considering the transverse strain-stress constitutive behavior of $\varepsilon_{z z}(\alpha, \beta, z)$ expressed in Eq. (C14) of Appendix, neglecting the converse piezoelectric effects, taking as approximations of the in-plane stress components the ones obtained with the FSDT for shells described in Eqs. (B5) of Appendix, i.e., $\sigma_{\alpha \alpha}^{*}(\alpha, \beta, z), \sigma_{\beta \beta}^{*}(\alpha, \beta, z)$ and $\sigma_{\alpha \beta}^{*}(\alpha, \beta, z)$, and considering $\sigma_{z z}(\alpha, \beta, z)$ as defined in (6), the transverse strain component is given by

$$
\varepsilon_{z z}(\alpha, \beta, z) \approx \bar{s}_{13} \sigma_{\alpha \alpha}^{*}(\alpha, \beta, z)+\bar{s}_{23} \sigma_{\beta \beta}^{*}(\alpha, \beta, z)+\bar{s}_{33} \sigma_{z z}(\alpha, \beta, z)+\bar{s}_{36} \sigma_{\alpha \beta}^{*}(\alpha, \beta, z) .
$$




\section{Mixed Displacement Field and Strains}

In this section the displacement field of the shell layer is derived by considering the out-of-plane strains $\varepsilon_{\beta z}$, $\varepsilon_{z \alpha}$ and $\varepsilon_{z z}$ presented in the previous section in Eqs. (17) and (18), respectively, and the out-of-plane straindisplacement relations in the third to fifth equations of (A1) in Appendix. By virtue of the assumptions previously considered,

$$
\begin{aligned}
\varepsilon_{z z} & =\frac{\partial w}{\partial z} \approx \bar{s}_{13}\left[\sigma_{\alpha \alpha}^{*(0)}(\alpha, \beta, z)+\sigma_{\alpha \alpha}^{*(1)}(\alpha, \beta, z)\right]+\bar{s}_{23}\left[\sigma_{\beta \beta}^{*(0)}(\alpha, \beta, z)+\sigma_{\beta \beta}^{*(1)}(\alpha, \beta, z)\right] \\
& +\bar{s}_{33}\left[\sigma_{z z}^{(0)}(\alpha, \beta, z)+\sigma_{z z}^{(1)}(\alpha, \beta, z)+\sigma_{z z}^{(2)}(\alpha, \beta, z)+\sigma_{z z}^{(f)}(\alpha, \beta, z)\right]+\bar{s}_{36}\left[\sigma_{\alpha \beta}^{*(0)}(\alpha, \beta, z)+\sigma_{\alpha \beta}^{*(1)}(\alpha, \beta, z)\right] .
\end{aligned}
$$

Thus, integrating the previous equation with respect to $z$ over the limits from 0 to $z$, considering that when $z=0$ we have $w(\alpha, \beta, z)=w_{0}(\alpha, \beta)$, and taking into account the time dependence of the strains and stresses definitions, the transverse displacement $w=w(\alpha, \beta, z, t)$ is given by

$$
w(\alpha, \beta, z, t)=w^{(0)}(\alpha, \beta, t)+w^{(1)}(\alpha, \beta, z, t)+w^{(2)}(\alpha, \beta, z, t)+w^{(3)}(\alpha, \beta, z, t)+w^{(f)}(\alpha, \beta, z, t),
$$

where

$$
\begin{aligned}
w^{(0)}(\alpha, \beta, t) & =w_{0}(\alpha, \beta, t), \\
w^{(1)}(\alpha, \beta, z, t) & =\int_{0}^{z}\left[\bar{s}_{13} \sigma_{\alpha \alpha}^{*(0)}(\alpha, \beta, z, t)+\bar{s}_{23} \sigma_{\beta \beta}^{*(0)}(\alpha, \beta, z, t)+\bar{s}_{33} \sigma_{z z}^{(0)}(\alpha, \beta, z, t)+\bar{s}_{36} \sigma_{\alpha \beta}^{*(0)}(\alpha, \beta, z, t)\right] d z, \\
w^{(2)}(\alpha, \beta, z, t) & =\int_{0}^{z}\left[\bar{s}_{13} \sigma_{\alpha \alpha}^{*(1)}(\alpha, \beta, z, t)+\bar{s}_{23} \sigma_{\beta \beta}^{*(1)}(\alpha, \beta, z, t)+\bar{s}_{33} \sigma_{z z}^{(1)}(\alpha, \beta, z, t)+\bar{s}_{36} \sigma_{\alpha \beta}^{*(1)}(\alpha, \beta, z, t)\right] d z, \\
w^{(3)}(\alpha, \beta, z, t) & =\int_{0}^{z} \bar{s}_{33} \sigma_{z z}^{(2)}(\alpha, \beta, z, t) d z, \\
w^{(f)}(\alpha, \beta, z, t) & =\int_{0}^{z} \bar{s}_{33} \sigma_{z z}^{(f)}(\alpha, \beta, z, t) d z .
\end{aligned}
$$

In a similar way, using the relations

$$
\begin{aligned}
& \varepsilon_{\beta z}(\alpha, \beta, z)=H_{\beta} \frac{\partial}{\partial z}\left(\frac{v(\alpha, \beta, z)}{H_{\beta}}\right)+\frac{1}{H_{\beta}} \frac{\partial w(\alpha, \beta, z)}{\partial \beta} \approx \frac{1}{H_{\beta}}\left[\varepsilon_{\beta z}^{(0)}(\alpha, \beta)+z \varepsilon_{\beta z}^{(1)}(\alpha, \beta)+\varepsilon_{\beta z}^{(f)}(\alpha, \beta, z)\right], \\
& \varepsilon_{z \alpha}(\alpha, \beta, z)=H_{\alpha} \frac{\partial}{\partial z}\left(\frac{u(\alpha, \beta, z)}{H_{\alpha}}\right)+\frac{1}{H_{\alpha}} \frac{\partial w(\alpha, \beta, z)}{\partial \alpha} \approx \frac{1}{H_{\alpha}}\left[\varepsilon_{z \alpha}^{(0)}(\alpha, \beta)+z \varepsilon_{z \alpha}^{(1)}(\alpha, \beta)+\varepsilon_{z \alpha}^{(f)}(\alpha, \beta, z)\right],
\end{aligned}
$$

integrating in order to $z$ over the limits from 0 to $z$ and considering that for $z=0$, the displacements on the middle surface are given by $u(\alpha, \beta, z)=u_{0}(\alpha, \beta)$ and $v(\alpha, \beta, z)=v_{0}(\alpha, \beta)$, the time dependent tangential displacements of any point of the shell are given by

$$
\begin{aligned}
u(\alpha, \beta, z, t) & =-H_{\alpha} \int_{0}^{z} \frac{1}{H_{\alpha}{ }^{2}} \frac{\partial w(\alpha, \beta, z, t)}{\partial \alpha} d z+H_{\alpha} \int_{0}^{z} \frac{1}{H_{\alpha}{ }^{2}}\left[\varepsilon_{z \alpha}^{(0)}(\alpha, \beta, t)+z \varepsilon_{z \alpha}^{(1)}(\alpha, \beta, t)+\varepsilon_{z \alpha}^{(f)}(\alpha, \beta, z, t)\right] d z \\
& =u^{(0)}(\alpha, \beta, z, t)+u^{(1)}(\alpha, \beta, z, t)+u^{(2)}(\alpha, \beta, z, t)+u^{(3)}(\alpha, \beta, z, t) \\
& +u^{(4)}(\alpha, \beta, z, t)+u^{(f)}(\alpha, \beta, z, t), \\
v(\alpha, \beta, z, t) & =-H_{\beta} \int_{0}^{z} \frac{1}{H_{\beta}{ }^{2}} \frac{\partial w(\alpha, \beta, z, t)}{\partial \beta} d z+H_{\beta} \int_{0}^{z} \frac{1}{H_{\beta}{ }^{2}}\left[\varepsilon_{\beta z}^{(0)}(\alpha, \beta, t)+z \varepsilon_{\beta z}^{(1)}(\alpha, \beta, t)+\varepsilon_{\beta z}^{(f)}(\alpha, \beta, z, t)\right] d z \\
& =v^{(0)}(\alpha, \beta, z, t)+v^{(1)}(\alpha, \beta, z, t)+v^{(2)}(\alpha, \beta, z, t)+v^{(3)}(\alpha, \beta, z, t) \\
& +v^{(4)}(\alpha, \beta, z, t)+v^{(f)}(\alpha, \beta, z, t),
\end{aligned}
$$


where

$$
\begin{aligned}
& u^{(0)}(\alpha, \beta, z, t)=\frac{H_{\alpha}}{A_{\alpha}} u_{0}(\alpha, \beta, t), \\
& u^{(1)}(\alpha, \beta, z, t)=-H_{\alpha} \int_{0}^{z} \frac{1}{H_{\alpha}^{2}}\left[\frac{\partial w^{(0)}(\alpha, \beta, t)}{\partial \alpha}-\varepsilon_{z \alpha}^{(0)}(\alpha, \beta, t)\right] d z, \\
& u^{(2)}(\alpha, \beta, z, t)=-H_{\alpha} \int_{0}^{z} \frac{1}{H_{\alpha}^{2}}\left[\frac{\partial w^{(1)}(\alpha, \beta, z, t)}{\partial \alpha}-z \varepsilon_{z \alpha}^{(1)}(\alpha, \beta, t)\right] d z, \\
& u^{(3)}(\alpha, \beta, z, t)=-H_{\alpha} \int_{0}^{z} \frac{1}{H_{\alpha}^{2}} \frac{\partial w^{(2)}(\alpha, \beta, z, t)}{\partial \alpha} d z, \\
& u^{(4)}(\alpha, \beta, z, t)=-H_{\alpha} \int_{0}^{z} \frac{1}{H_{\alpha}^{2}} \frac{\partial w^{(3)}(\alpha, \beta, z, t)}{\partial \alpha} d z, \\
& u^{(f)}(\alpha, \beta, z, t)=-H_{\alpha} \int_{0}^{z} \frac{1}{H_{\alpha}^{2}}\left[\frac{\partial w^{(f)}(\alpha, \beta, z, t)}{\partial \alpha}-\varepsilon_{z \alpha}^{(f)}(\alpha, \beta, z, t)\right] d z, \\
& v^{(0)}(\alpha, \beta, z, t)=\frac{H_{\beta}}{A_{\beta}} v_{0}(\alpha, \beta, t) \\
& v^{(1)}(\alpha, \beta, z, t)=-H_{\beta} \int_{0}^{z} \frac{1}{H_{\beta}^{2}}\left[\frac{\partial w^{(0)}(\alpha, \beta, t)}{\partial \beta}-\varepsilon_{\beta z}^{(0)}(\alpha, \beta, t)\right] d z, \\
& v^{(2)}(\alpha, \beta, z, t)=-H_{\beta} \int_{0}^{z} \frac{1}{H_{\beta}^{2}}\left[\frac{\partial w^{(1)}(\alpha, \beta, z, t)}{\partial \beta}-z \varepsilon_{\beta z}^{(1)}(\alpha, \beta, t)\right] d z, \\
& v^{(3)}(\alpha, \beta, z, t)=-H_{\beta} \int_{0}^{z} \frac{1}{H_{\beta}^{2}} \frac{\partial w^{(2)}(\alpha, \beta, z, t)}{\partial \beta} d z, \\
& v^{(4)}(\alpha, \beta, z, t)=-H_{\beta} \int_{0}^{z} \frac{1}{H_{\beta}^{2}} \frac{\partial w^{(3)}(\alpha, \beta, z, t)}{\partial \beta} d z, \\
& v^{(f)}(\alpha, \beta, z, t)=-H_{\beta} \int_{0}^{z} \frac{1}{H_{\beta}^{2}}\left[\frac{\partial w^{(f)}(\alpha, \beta, z, t)}{\partial \beta}-\varepsilon_{\beta z}^{(f)}(\alpha, \beta, z, t)\right] d z,
\end{aligned}
$$

Eqs. (20), (23) and (24) show that in comparison with the CLT of shells, following Love's first approximation, and the FSDT of shells discussed in Appendix B, the in-plane and transverse displacements of any point of the shell are nonlinearly dependent on $z$. Additionally, the same 5 time dependent generalized displacements of the FSDT (see Appendix B), $u_{0}=u_{0}(\alpha, \beta, t), v_{0}=v_{0}(\alpha, \beta, t)$ and $w_{0}=w_{0}(\alpha, \beta, t)$, which are are the tangential and transverse displacements referred to a point on the middle surface, respectively, and the shear angle rotations of a normal to the reference middle surface,

$$
\begin{aligned}
& \psi_{\alpha}(\alpha, \beta, t)=\frac{\partial w_{0}(\alpha, \beta, t)}{\partial \alpha}+A_{\alpha}(\alpha, \beta) \theta_{\alpha}(\alpha, \beta, t)-\frac{A_{\alpha}(\alpha, \beta)}{R_{\alpha}(\alpha, \beta)} u_{0}(\alpha, \beta, t), \\
& \psi_{\beta}(\alpha, \beta, t)=\frac{\partial w_{0}(\alpha, \beta, t)}{\partial \beta}+A_{\beta}(\alpha, \beta) \theta_{\beta}(\alpha, \beta, t)-\frac{A_{\beta}(\alpha, \beta)}{R_{\beta}(\alpha, \beta)} v_{0}(\alpha, \beta, t),
\end{aligned}
$$

are used to define the "generalized" displacements of the proposed refined theory which represent non-linear functions in $z$ of the generalized displacements of FSDT. Thus, based on the assumptions (b)-(d) the 3-D problem of the theory of elasticity has been fully brought to a 2-D problem of the theory of the shell, with Eqs. (20), (23) and (24) establishing the geometrical model of the deformed state of the fully refined theory of the generic shell layer.

On the basis of the refined displacement field defined by Eqs. (20), (23) and (24) and the general straindisplacement relations in Eqs. (A1) in Appendix, the not yet defined in-plane strain components of the proposed theory may be determined. For the sake of brevity their definitions will not be given here since they are quite long equations, in terms of high-order derivatives of the generalized displacements of the FSDT, which can be derived from the previous definitions. 


\section{E. Electric Field and Potential}

Resuming the basic hypotheses defined in Sec. C, and regarding the electrical and electro-mechanical behavior, the following considerations and assumptions regarding the physical problem are made:

(e) The piezoelectric layers are polarized in the thickness direction and electroded only in the top and bottom surfaces;

(f) Only a transverse electric field $\hat{E}_{z}$ is externally applied;

(g) The in-plane electric displacement components $D_{x}$ and $D_{y}$ are nil at the non-electroded lateral edges and are considered negligible inside the piezoelectric volume;

(h) The out-of-plane component of the electric displacement field $D_{z}$ is constant with respect to the thickness;

The behavior of the electromagnetic field is described by Maxwell's equations. However, as presented by Tiersten ${ }^{43}$ considering that the electromagnetic waves essentially uncouple from the elastic waves and that the elastic wavelengths are much shorter than the electromagnetic ones at the same frequency, Maxwell's equations can be replaced by electrostatic equations in vector form,

$$
\begin{aligned}
\operatorname{div} \mathbf{D} & =0, \\
-\operatorname{grad} \varphi & =\mathbf{E},
\end{aligned}
$$

where $\varphi=\varphi(\alpha, \beta, z)$ is the electric potential, and $\mathbf{D}=\mathbf{D}(\alpha, \beta, z)$ and $\mathbf{E}=\mathbf{E}(\alpha, \beta, z)$ are the electric displacement and field vectors. In scalar form, according to the definitions of the divergence and gradient operators in arbitrary orthogonal curvilinear coordinates (see Ref. 105) and Eqs. (2), the electrostatic equations are given as

$$
\begin{gathered}
\frac{1}{H_{\alpha} H_{\beta}}\left[\frac{\partial}{\partial \alpha}\left(H_{\beta} D_{\alpha}\right)+\frac{\partial}{\partial \beta}\left(H_{\alpha} D_{\beta}\right)+\frac{\partial}{\partial z}\left(H_{\alpha} H_{\beta} D_{z}\right)\right]=0, \\
E_{\alpha}=-\frac{1}{H_{a}} \frac{\partial \varphi}{\partial \alpha}, \quad E_{\beta}=-\frac{1}{H_{\beta}} \frac{\partial \varphi}{\partial \beta}, \quad E_{z}=-\frac{\partial \varphi}{\partial z} .
\end{gathered}
$$

According to what has bee discussed in Appendix C, for the present problem the electrodes are located on the top and bottom surfaces and only a transverse electric field $\hat{E}_{z}$ is externally applied. The conditions $D_{\alpha}=D_{\beta}=0$ hold in the shell surfaces not covered with electrodes and in contact with a medium with low permittivity, e.g., vacuum or air (see Refs. 40,42 and 45 for further details) and the induced electric fields $\bar{E}_{\alpha}$ and $\bar{E}_{\beta}$ (which are defined as a function of the strains) where condensed in the constitutive Eq. (C10). Thus, Eq. (30) can be written as

$$
\frac{1}{H_{\alpha} H_{\beta}}\left[\frac{\partial}{\partial z}\left(H_{\alpha} H_{\beta} D_{z}\right)\right]=0 .
$$

In view of the Gauss and Mainardi-Codazzi formulas (cf. Refs. 4 and 45),

$$
\frac{\partial H_{\alpha}}{\partial \beta}=\left(1+\frac{z}{R_{\beta}}\right) \frac{\partial A_{\alpha}}{\partial \beta}, \quad \frac{\partial H_{\beta}}{\partial \alpha}=\left(1+\frac{z}{R_{a}}\right) \frac{\partial A_{\beta}}{\partial \alpha}, \quad \frac{\partial H_{\alpha}}{\partial z}=\frac{A_{\alpha}}{R_{\alpha}}, \quad \frac{\partial H_{\beta}}{\partial z}=\frac{A_{\beta}}{R_{\beta}},
$$

after some algebra, and taking into account Eqs. (2), Eq. (32) is expressed as

$$
\left(\frac{1}{R_{\alpha}+z}+\frac{1}{R_{\beta}+z}\right) D_{z}+\frac{\partial D_{z}}{\partial z}=0 .
$$

Assuming a constant value of $\alpha$ and $\beta$, the previous partial differential equation can be solved as an ordinary differential equation in $z$ with solution

$$
D_{z}=C(\alpha, \beta) \frac{\left(-R_{\alpha}+h\right)\left(-R_{\beta}+h\right)}{\left(R_{\alpha}+h\right)\left(R_{\beta}+h\right)}=C(\alpha, \beta) \lambda(\alpha, \beta) .
$$

From Eq. (35) it can be seen that the transverse component of the electric displacement is constant with respect to the thickness direction where the constant $\lambda(\alpha, \beta)$ is a correction factor that takes into account 
the effects of the radii of curvature (and half thickness of the layer) on the electric displacement. Thus, taking into account (C11) of Appendix, it can be reformulated ${ }^{40-42}$ taking into account the curvature effects via $\lambda(\alpha, \beta)$ as

$$
D_{z}=\frac{\lambda}{2 h} \int_{-h}^{+h} \bar{\epsilon}_{33}\left(\hat{E}_{z}-\bar{E}_{z}\right) d z=-\lambda \bar{\epsilon}_{33} \frac{\phi}{2 h}-\frac{\lambda}{2 h} \bar{\epsilon}_{33} \int_{-h}^{+h} \bar{E}_{z} d z,
$$

In the previous equation the prescribed transverse electric field component has been defined as $\hat{E}_{z}=-\phi /(2 h)$ where $\phi=\phi(\alpha, \beta)$ denotes the electric potential difference between the top and bottom electrodes. Considering the previous equation and the general electrostatic constitutive behavior in $(\mathrm{C} 2)$, the net transverse electric field $E_{z}$ is given by the sum of the effects of the externally applied electric potential difference $\phi$ and net induced term which depends of the strains $\tilde{E}_{z}$, and is expressed as

$$
E_{z}=\lambda\left(\hat{E}_{z}+\tilde{E}_{z}\right)=-\lambda \frac{\phi}{2 h}+\left(\lambda \bar{E}_{z}-\frac{\lambda}{2 h} \int_{-h}^{+h} \bar{E}_{z} d z\right)
$$

It is worthy to mention that the terms inside parenthesis in the previous equation are related with the mechanical strains and represent the net effects of the induced electric fields due to the direct piezoelectric effect. Finally, from the definition of the electric potential in the third equation of (31), substituting (37) and integrating with respect to the thickness of the layer, the through-the-thickness distribution of the electric potential is given by

$$
\varphi=\lambda \frac{\phi}{2 h}(z+h)+\left(\frac{\lambda}{2 h} \int_{-h}^{+h} \bar{E}_{z} d z\right)(z+h)-\lambda \int_{-h}^{z} \bar{E}_{z} d z .
$$

It can be seen that the net transverse electric field $E_{z}=E_{z}(\alpha, \beta, z)$ and electric potential distribution $\varphi=\varphi(\alpha, \beta, z)$ for the curvilinear coordinate system presented in Eqs. (37) and (38) are composed by terms externally applied and by induced terms due to the mechanical deformations. Furthermore, the formulation is kept general, since the induced terms are not explicitly defined at this point, and can be applied for any piezoelectric curved layer (and obviously for 1-D and 2-D planar structures where the term $\lambda$ is equal to unity).

\section{Partially Refined Mathematical Model of Doubly-Curved Shells}

\section{A. Restrictions and Simplifications}

The definitions of the displacement field presented in Eqs. (20), (23) and (24) are quite general and applicable to anisotropic shells of arbitrary curvature. They result from a fully refined interactive shell theory based, as a first approximation, on the in-plane stresses of the FSDT. Furthermore, all the terms regarding the thickness coordinate to radii or curvature ratios were retained and no simplifications were made regarding thin shell assumptions. Additionally, transverse shear strains and stresses weren't considered negligible and as a result a non-linearly dependent on $z$ transverse displacement was obtained by the iterative procedure. That theory was denoted as fully refined since all the strain and stress components of the 3-D elasticity are obtained directly from the mixed (in terms of surface stresses and generalized displacements) displacement field by using the strain-displacement relations and an anisotropic constitutive law. It can also be seen from the definitions of the terms of the in-plane displacements in Eqs. (25) and (26) that they involve high-order derivatives (which become even higher for the strain and stress components) of the generalized displacements of the FSDT, which complicates the formulation and FE solution dramatically. Additionally, the fully refined mathematical model allows full out-of-plane interlayer (or interlaminar) stress continuity (and, as obvious, displacements too) to be imposed when assembling all the layers contributions at the "regenerated" FE level (further details will be discussed later). This renders a 2-D theory representative of the full 3-D behavior of a shell with arbitrary geometry (curvature).

However, the fully refined mixed displacement definitions are quite tedious and, for the sake of making the calculations less cumbersome, the general mixed displacement field definitions will be restricted to orthotropic shells with constant curvatures, i.e., doubly-curved shells (cylindrical, spherical, toroidal geometries) for which

$$
A_{\alpha}=A_{\beta}=1, \quad \frac{\partial A_{\alpha}}{\partial \beta}=\frac{\partial A_{\beta}}{\partial \alpha}=0, \quad R_{\alpha}(\alpha, \beta)=R_{\alpha}, \quad R_{\beta}(\alpha, \beta)=R_{\beta}
$$


Additionally, the non-linear transverse displacement field definition will be discarded and only the zero-order term, i.e., $w(\alpha, \beta, z, t)=w^{(0)}(\alpha, \beta, t)$, will be retained. This simplification makes the theory less complicated and more suitable to be implemented, since it avoids the higher-order derivatives of the generalized variables, and is denoted as partial refined theory. It is well known, however, that in the major part of the problems, the transverse stress is small when compared with the other stress components. An exception is, for example, in thermo-mechanical analysis where the transverse stress $\sigma_{z z}$ plays an important role (see Carrera ${ }^{8,106}$ ), which is not the case here. This simplification implies also the need to make the usual plane-stress assumption, which doesn't allow to impose interlayer transverse normal stress continuity. Regarding the shear stress correction function $f(z)$, several functions can be used. However, as stated by Ambartsumian [23, p. 37], some arbitrariness in the reasonable selection of $f(z)$ will not introduce inadmissible errors into the refined theory, which in this work will be assumed to follow the law of a quadratic parabola as

$$
f(z)=1-\frac{z^{2}}{h^{2}}
$$

\section{B. Displacements and Strains}

For the sake of simplicity and brevity of writing the mathematical definitions, from this point henceforth, the spatial $(\alpha, \beta, z)$ and time $t$ dependencies will be omitted from the equations when convenient and only written when necessary for the comprehension of the equations. Thus, under the doubly-curved shell restrictions to the general problem, and following the previously defined partial refined theory, the displacement definitions in Eqs. (20), (23) and (24), for an orthotropic shell layer, taking into account the definitions in (B3) of the Appendix, are given as

$$
\begin{aligned}
u(\alpha, \beta, z, t) & =\frac{1}{z_{\alpha}^{(0)}}\left[u_{0}+z_{\alpha}^{*(f)} \bar{s}_{55}^{*} \psi_{\alpha}-z_{\alpha}^{*(0)} \frac{\partial w_{0}}{\partial \alpha}+z_{\alpha}^{*(0)} \bar{s}_{55}^{*} \bar{\tau}_{z \alpha}+z_{\alpha}^{*(1)} \frac{\bar{s}_{55}^{*}}{2 h} \tilde{\tau}_{z \alpha}\right], \\
v(\alpha, \beta, z, t) & =\frac{1}{z_{\beta}^{(0)}}\left[v_{0}+z_{\beta}^{*(f)} \bar{s}_{44}^{*} \psi_{\beta}-z_{\beta}^{*(0)} \frac{\partial w_{0}}{\partial \beta}+z_{\beta}^{*(0)} \bar{s}_{44}^{*} \bar{\tau}_{\beta z}+z_{\beta}^{*(1)} \frac{\bar{s}_{44}^{*}}{2 h} \tilde{\tau}_{\beta z}\right], \\
w(\alpha, \beta, z, t) & =w_{0},
\end{aligned}
$$

where $z_{\alpha}^{(0)}$ and $z_{\beta}^{(0)}$ are defined in Eqs. (B3) of Appendix and

$$
\begin{array}{lll}
z_{\alpha}^{*(i)}=\int_{0}^{z} \frac{z^{i}}{\left(1+z / R_{\alpha}\right)^{2}} d z, & z_{\beta}^{*(i)}=\int_{0}^{z} \frac{z^{i}}{\left(1+z / R_{\beta}\right)^{2}} d z \\
z_{\alpha}^{*(f)}=\int_{0}^{z} \frac{f(z)}{\left(1+z / R_{\alpha}\right)^{2}} d z, & z_{\beta}^{*(f)}=\int_{0}^{z} \frac{f(z)}{\left(1+z / R_{\beta}\right)^{2}} d z
\end{array}
$$

with $i=0,1$. As can be seen in the mixed displacement field definition in Eqs. (41), the displacement field is defined in terms of the generalized displacements of the FSDT and mean and relative surface shear stresses. Additionally, as would be expected, if $f(z)$ is assumed equal to one, which corresponds to case where no correction to the FSDT constant through-the-thickness shear stresses/strains is made, the displacement is the same as the one obtained with the FSDT, with the extra surface shear stress terms. In the limit case where $R_{\alpha}=R_{\beta}=\infty$, which corresponds to the case of planar structures such as plates, and considering the parabolic definition of $f(z)$, the displacements are consistent and are expanded in a power series of $z$ up to $z^{3}$. In the present case, since the terms $z / R_{\alpha}$ and $z / R_{\beta}$ were fully retained, the displacements are defined with more complex coefficients in terms of powers of $z$ and $\ln \left(R_{\alpha}+z\right)$ and $\ln \left(R_{\beta}+z\right)$. For convenience, the mixed displacement field in Eqs. (41) can be expressed in matrix form as

$$
\mathbf{u}(\alpha, \beta, z, t)=\mathbf{z}^{u}(z) \mathbf{u}_{0}(\alpha, \beta, t)+\mathbf{z}^{\tau}(z) \boldsymbol{\tau}(\alpha, \beta, t),
$$

or, alternatively,

$$
\left\{\begin{array}{l}
u \\
v \\
w
\end{array}\right\}=\left[\begin{array}{ccccc}
z_{11}^{u} & 0 & z_{13}^{u} \partial_{\alpha} & z_{14}^{u} & 0 \\
0 & z_{22}^{u} & z_{23}^{u} \partial_{\beta} & 0 & z_{25}^{u} \\
0 & 0 & 1 & 0 & 0
\end{array}\right]\left\{\begin{array}{c}
u_{0} \\
v_{0} \\
w_{0} \\
\theta_{\alpha} \\
\theta_{\beta}
\end{array}\right\}+\left[\begin{array}{cccc}
z_{11}^{\tau} & z_{12}^{\tau} & 0 & 0 \\
0 & 0 & z_{23}^{\tau} & z_{24}^{\tau} \\
0 & 0 & 0 & 0
\end{array}\right]\left\{\begin{array}{c}
\bar{\tau}_{z \alpha} \\
\tilde{\tau}_{z \alpha} \\
\bar{\tau}_{\beta z} \\
\tilde{\tau}_{\beta z}
\end{array}\right\}
$$


where the coefficients of matrices $\mathbf{z}^{u}(z)$ and $\mathbf{z}^{\tau}(z)$ are derived from the displacements as explained in Appendix D. In the specific case of $z_{13}^{u}(z)$ and $z_{23}^{u}(z)$, which are related with $\partial w_{0} / \partial \alpha$ and $\partial w_{0} / \partial \beta$, partial derivative operators $\partial_{\alpha}$ and $\partial_{\beta}$ in order to $\alpha$ and $\beta$, respectively, where also included.

From the definition of the displacement field in Eq. (43), the out-of-plane shear strains are given by Eqs. (17), taking into account the restrictions in (39) and the transverse strain $\varepsilon_{z z}=0$ [due to the fact that $w(\alpha, \beta, z, t)$ was assumed independent of $z]$. The in-plane strain components are obtained according to the displacement-strain relations in the first two and last equations of Eqs. (A1) by taking into account (39). Thus, the strains vector without the null component $\varepsilon_{z z}$ may be expressed in matrix form as

$$
\begin{aligned}
\varepsilon(\alpha, \beta, z, t) & =\partial_{\varepsilon}(z) \mathbf{z}^{u}(z) \mathbf{u}_{0}(\alpha, \beta, t)+\partial_{\varepsilon}(z) \mathbf{z}^{\tau}(z) \boldsymbol{\tau}(\alpha, \beta, t) \\
& =\mathbf{z}^{\varepsilon u}(z) \mathbf{u}_{0}(\alpha, \beta, t)+\mathbf{z}^{\varepsilon \tau}(z) \boldsymbol{\tau}(\alpha, \beta, t),
\end{aligned}
$$

where $\partial_{\varepsilon}(z)$ is a matrix differential operator given by

$$
\partial_{\varepsilon}(z)=\left[\begin{array}{ccc}
\partial_{\alpha} z_{\alpha}^{(0)} & 0 & z_{\alpha}^{(0)} / R_{\alpha} \\
0 & \partial_{\beta} z_{\beta}^{(0)} & z_{\beta}^{(0)} / R_{\beta} \\
0 & \partial_{z}-z_{\beta}^{(0)} / R_{\beta} & \partial_{\beta} z_{\beta}^{(0)} \\
\partial_{z}-z_{\alpha}^{(0)} / R_{\alpha} & 0 & \partial_{\alpha} z_{\alpha}^{(0)} \\
\partial_{\beta} z_{\beta}^{(0)} & \partial_{\alpha} z_{\alpha}^{(0)} & 0
\end{array}\right],
$$

and $\partial_{z}$ is an other partial differential operator, this time in order to $z$. Considering the previous operator matrix in Eq. (45), the strains vector are defined in terms of the matrices $\mathbf{z}^{\varepsilon u}(z)$ and $\mathbf{z}^{\varepsilon \tau}(z)$ as

$$
\begin{aligned}
\left\{\begin{array}{c}
\varepsilon_{\alpha \alpha} \\
\varepsilon_{\beta \beta} \\
\varepsilon_{\beta z} \\
\varepsilon_{z \alpha} \\
\varepsilon_{\alpha \beta}
\end{array}\right\}= & {\left[\begin{array}{ccccc}
z_{11}^{\varepsilon u} \partial_{\alpha} & 0 & z_{13}^{\varepsilon u 1}+z_{13}^{\varepsilon u 2} \partial_{\alpha \alpha} & z_{14}^{\varepsilon u} \partial_{\alpha} & 0 \\
0 & z_{22}^{\varepsilon u} \partial_{\beta} & z_{23}^{\varepsilon u 1}+z_{23}^{\varepsilon u 2} \partial_{\beta \beta} & 0 & z_{25}^{\varepsilon u} \partial_{\beta} \\
0 & z_{32}^{\varepsilon u} & z_{33}^{\varepsilon u} \partial_{\beta} & 0 & z_{35}^{\varepsilon u} \\
z_{41}^{\varepsilon u} & 0 & z_{43}^{\varepsilon u} \partial_{\alpha} & z_{44}^{\varepsilon u} & 0 \\
z_{51}^{\varepsilon u} \partial_{\beta} & z_{52}^{\varepsilon u} \partial_{\alpha} & z_{53}^{\varepsilon u} \partial_{\alpha \beta} & z_{54}^{\varepsilon u} \partial_{\beta} & z_{55}^{\varepsilon u} \partial_{\alpha}
\end{array}\right]\left\{\begin{array}{c}
u_{0} \\
v_{0} \\
w_{0} \\
\theta_{\alpha} \\
\theta_{\beta}
\end{array}\right\} } \\
+ & {\left[\begin{array}{cccc}
z_{11}^{\varepsilon \tau} \partial_{\alpha} & z_{12}^{\varepsilon \tau} \partial_{\alpha} & 0 & 0 \\
0 & 0 & z_{23}^{\varepsilon \tau} \partial_{\beta} & z_{24}^{\varepsilon \tau} \partial_{\beta} \\
0 & 0 & z_{33}^{\varepsilon \tau} & z_{34}^{\varepsilon \tau} \\
z_{41}^{\varepsilon \tau} & z_{42}^{\varepsilon \tau} & 0 & 0 \\
z_{51}^{\varepsilon \tau} \partial_{\beta} & z_{52}^{\varepsilon \tau} \partial_{\beta} & z_{53}^{\varepsilon \tau} \partial_{\alpha} & z_{54}^{\varepsilon \tau} \partial_{\alpha}
\end{array}\right]\left\{\begin{array}{c}
\bar{\tau}_{z \alpha} \\
\tilde{\tau}_{z \alpha} \\
\bar{\tau}_{\beta z} \\
\tilde{\tau}_{\beta z}
\end{array}\right\} }
\end{aligned}
$$

where $\partial_{\alpha \alpha}, \partial_{\beta \beta}$ and $\partial_{\alpha \beta}$ are double and crossed partial differential operators. The coefficients of $\mathbf{z}^{\varepsilon u}(z)$ and $\mathbf{z}^{\varepsilon \tau}(z)$ are given in Appendix D. It is worthy to mention that the strain field is defined in terms of zeroand/or first-order derivatives of the generalized in-plane displacements, rotations and surface stresses, and zero-, second-order and cross derivatives of the generalized transverse displacement $w_{0}$.

\section{Induced Electric Field and Net Strain Field}

The net electric field $E_{z}$ in Eq. (37) is expressed in terms of external prescribed and induced effects. Considering the electric eigenfield definition in the third equation of Eqs. (C8), and since $\varepsilon_{z z}=0$, the net induced term $\tilde{E}_{z}$ for the orthotropic shell layer is given by

$$
\tilde{E}_{z}=\frac{\lambda}{\bar{\epsilon}_{33}^{*}}\left[-\bar{e}_{31}^{*} \varepsilon_{\alpha \alpha}-\bar{e}_{32}^{*} \varepsilon_{\beta \beta}+\frac{1}{2 h} \int_{-h}^{+h}\left(\bar{e}_{31}^{*} \varepsilon_{\alpha \alpha}+\bar{e}_{32}^{*} \varepsilon_{\beta \beta}\right) d z\right],
$$

where $\bar{e}_{31}^{*}$ and $\bar{e}_{32}^{*}$ are the reduced (modified) piezoelectric plane-stress constants.

Considering the definitions of the in-plane strain components $\varepsilon_{\alpha \alpha}$ and $\varepsilon_{\beta \beta}$ given in Eq. (47), the previous equation can be expressed in terms of the generalized displacements, and after some algebra, the net strain field accounting for the direct piezoelectric effects taking into account the plane-stress version of the electrical 
relation of Eq. (C14), is given by

$$
\begin{aligned}
\left\{\begin{array}{c}
\varepsilon_{\alpha \alpha} \\
\varepsilon_{\beta \beta} \\
\varepsilon_{\beta z} \\
\varepsilon_{z \alpha} \\
\varepsilon_{\alpha \beta}
\end{array}\right\}= & {\left[\begin{array}{ccccc}
z_{11}^{\star \varepsilon u} \partial_{\alpha} & z_{12}^{\star \varepsilon u} \partial_{\beta} & z_{13}^{\star \varepsilon u 1}+z_{13}^{\star \varepsilon u 2} \partial_{\alpha \alpha} & z_{14}^{\star \varepsilon u} \partial_{\alpha} & z_{15}^{\star \varepsilon u} \partial_{\beta} \\
z_{21}^{\star \varepsilon u} \partial_{\alpha} & z_{22}^{\star \varepsilon u} \partial_{\beta} & z_{23}^{\star \varepsilon u 1}+z_{23}^{\star \varepsilon u 2} \partial_{\beta \beta} & z_{24}^{\star \varepsilon u} \partial_{\alpha} & z_{25}^{\star \varepsilon u} \partial_{\beta} \\
0 & z_{32}^{\varepsilon u} & z_{33}^{\varepsilon u} \partial_{\beta} & 0 & z_{35}^{\varepsilon u} \\
z_{41}^{\varepsilon u} & 0 & z_{33}^{\varepsilon u} \partial_{\alpha} & z_{44}^{\varepsilon u} & 0 \\
z_{51}^{\varepsilon u} \partial_{\beta} & z_{52}^{\varepsilon u} \partial_{\alpha} & z_{53}^{\varepsilon u} \partial_{\alpha \beta} & z_{54}^{\varepsilon u} \partial_{\beta} & z_{55}^{\varepsilon u} \partial_{\alpha}
\end{array}\right]\left\{\begin{array}{c}
u_{0} \\
v_{0} \\
w_{0} \\
\theta_{\alpha} \\
\theta_{\beta}
\end{array}\right\} } \\
+ & {\left[\begin{array}{cccc}
z_{11}^{\star \varepsilon \tau} \partial_{\alpha} & z_{12}^{\star \varepsilon \tau} \partial_{\alpha} & z_{13}^{\star \varepsilon \tau} \partial_{\beta} & z_{14}^{\star \varepsilon \tau} \partial_{\beta} \\
z_{21}^{\star \varepsilon \tau} \partial_{\alpha} & z_{22}^{\star \varepsilon \tau} \partial_{\alpha} & z_{23}^{\star \varepsilon \tau} \partial_{\beta} & z_{24}^{\star \varepsilon \tau} \partial_{\beta} \\
0 & 0 & z_{33}^{\varepsilon \tau} & z_{34}^{\varepsilon \tau} \\
z_{41}^{\varepsilon \tau} & z_{42}^{\varepsilon \tau} & 0 & 0 \\
z_{51}^{\varepsilon \tau} \partial_{\beta} & z_{52}^{\varepsilon \tau} \partial_{\beta} & z_{53}^{\varepsilon \tau} \partial_{\alpha} & z_{54}^{\varepsilon \tau} \partial_{\alpha}
\end{array}\right]\left\{\begin{array}{c}
\bar{\tau}_{z \alpha} \\
\tilde{\tau}_{z \alpha} \\
\bar{\tau}_{\beta z} \\
\tilde{\tau}_{\beta z}
\end{array}\right\}, }
\end{aligned}
$$

where the modified (taking into account the electrical induced strains) terms of $\mathbf{z}^{\varepsilon u}(z)$ and $\mathbf{z}^{\varepsilon \tau}(z)$ are given in Appendix E.

\section{Variational Formulation}

In order to derive the weak form of the equations governing the motion and electric charge equilibrium of the shell layer, Hamilton's principle is used. The Lagrangian and the work of the applied forces are adapted for the electrical and mechanical contributions, ${ }^{43}$ so that

$$
\delta \int_{t_{0}}^{t_{1}}(T-H+W) d t=0,
$$

where $t_{0}$ and $t_{1}$ define the time interval, $\delta$ denotes the variation, $T$ is the kinetic energy, $H$ is the electromechanical enthalpy and $W$ denotes the work of the external non-conservative actions done by the applied mechanical forces and electrical charges.

Since the stresses have been replaced and considered by means of internal forces and moments due to the thickness integration it is appropriate to alter the definition of the fundamental element of the shell. Accordingly, it will be assumed, henceforth, that the element which was formerly defined to be $d z$ thick, is replaced, on account of the integrations with respect to $z$, with an element of thickness $h$. Such an element is acted upon by the internal forces (stress resultants) and moments per unit arc length and by external effects which include mechanical forces and electrical charge loads. The internal forces act upon the edges of the element while the mechanical forces and electrical charges act upon the inner and outer surfaces/electrodes.

According to Eq. (43), the kinetic energy is given by

$$
T=\frac{1}{2} \int_{V} \rho \dot{\mathbf{u}}^{\mathrm{T}} \dot{\mathbf{u}} d V
$$

where $\dot{\mathbf{u}}=\dot{\mathbf{u}}(\alpha, \beta, z, t)$ is the vector of generalized velocities taking into account the time differentiation of the three components of the displacement field expressed in the tri-orthogonal curvilinear coordinate system. The first variation of the kinetic energy yields the virtual work of the inertial forces, given by

$$
\delta T=-\int_{\Omega_{0}}\left[\int_{-h}^{+h} \rho \delta \mathbf{u}^{\mathrm{T}} \ddot{\mathbf{u}} H_{\alpha} H_{\beta} H_{z} d z\right] d \alpha d \beta,
$$

which is expanded with more detail in terms of the variations of the generalized displacements and stresses in Appendix F1.

The electro-mechanical enthalpy of the piezoelectric medium is expressed in terms of mechanical and electrical quantities by

$$
H=\frac{1}{2} \int_{V}\left(\boldsymbol{\sigma}^{\mathrm{T}} \boldsymbol{\varepsilon}-\mathbf{D}^{\mathrm{T}} \mathbf{E}\right) d V .
$$


Taking into account the plane-stress piezoelectric constitutive Eq. (C15) in the Appendix, the first variation of the previous equation yields

$$
\begin{aligned}
\delta H & =\delta H_{\varepsilon}-\delta H_{E} \\
& =\int_{\Omega_{0}}\left[\int_{-h}^{+h} \boldsymbol{\sigma}^{\mathrm{T}} \delta \varepsilon H_{\alpha} H_{\beta} H_{z} d z-\int_{-h}^{+h} D_{z} \delta E_{z} H_{\alpha} H_{\beta} H_{z} d z\right] d \alpha d \beta,
\end{aligned}
$$

which is expanded with more detail in terms of the variations of the generalized displacements and internal forces and moments in Appendix F2.

The last term of Eq. (50) involves the work done by the applied mechanical forces $W_{u}$, applied on the inner and outer surfaces and lateral edges of the shell, and the work done by the applied electrical charges density $W_{\phi}$ on the top and bottom electroded surfaces. To write the expressions for the net external loads work recall that $\Omega$ denotes a surface at a distance $z$ and parallel to the middle-surface, where $\Omega^{t}$ and $\Omega^{b}$ denote the top and bottom surfaces for which $z= \pm h$, and that $\Gamma$ denotes the boundary of the shell element, with $\Gamma_{\alpha}$ and $\Gamma_{\beta}$ being the boundary edges of constant $\beta$ and $\alpha$ coordinates, respectively (with the circle on the integral implying that it includes the total boundary of the shell). Thus, the work of the non-conservative loads is given by

$$
W=W_{u}-W_{\phi} .
$$

The mechanical counterpart is given by

$$
\begin{aligned}
W_{u} & =\int_{\Omega^{t}} F_{z}^{t} w d \Omega^{t}+\int_{\Omega^{b}} F_{z}^{b} w d \Omega^{b}+\oint_{\Gamma_{\alpha}}\left[\int_{-h}^{+h}\left(\hat{\sigma}_{\beta \beta} v+\hat{\sigma}_{\beta \alpha} u+\hat{\sigma}_{\beta z} w\right) \frac{1}{z_{\alpha}^{(0)}} d z\right] d \alpha \\
& +\oint_{\Gamma_{\beta}}\left[\int_{-h}^{+h}\left(\hat{\sigma}_{\alpha \alpha} u+\hat{\sigma}_{\alpha \beta} v+\hat{\sigma}_{z \alpha} w\right) \frac{1}{z_{\beta}^{(0)}} d z\right] d \beta,
\end{aligned}
$$

where $F_{z}^{t}=F_{z}^{t}(\alpha, \beta, t)$ and $F_{z}^{b}=F_{z}^{b}(\alpha, \beta, t)$ are transverse normal forces applied on the top and bottom surfaces, and the hat over the stresses, $\hat{\sigma}_{\alpha \alpha}=\hat{\sigma}_{\alpha \alpha}(\beta, z, t), \hat{\sigma}_{\alpha \beta}=\hat{\sigma}_{\alpha \beta}(\beta, z, t)$ and $\hat{\sigma}_{z \alpha}=\hat{\sigma}_{z \alpha}(\beta, z, t)$, for the edges normal to $\alpha$, and $\hat{\sigma}_{\beta \beta}=\hat{\sigma}_{\beta \beta}(\alpha, z, t), \hat{\sigma}_{\beta \alpha}=\hat{\sigma}_{\beta \alpha}(\alpha, z, t)$ and $\hat{\sigma}_{\beta z}=\hat{\sigma}_{\beta z}(\alpha, z, t)$, for the edges normal to $\beta$, denotes prescribed stresses on the boundary edges. Regarding the electrical counterpart, it is given by

$$
W_{\phi}=\int_{\Omega^{t}} \tau^{t} \varphi d \Omega^{t}+\int_{\Omega^{b}} \tau^{b} \varphi d \Omega^{t},
$$

where $\tau^{t}=\tau^{t}(\alpha, \beta, t)$ and $\tau^{b}=\tau^{b}(\alpha, \beta, t)$ are prescribed electric charge densities on the top and bottom electroded surfaces.

Considering in Eq. (38) that the induced electric field has no contribution for the electric potential at the surface electrodes, assuming the bottom electrode grounded, i.e., $\varphi(\alpha, \beta, z=-h, t)=0$, and retaining only the normal mechanical load on the top surface $F_{z}^{t}$, the first variation of Eq. (54) yields the net virtual work of the non-conservative external effects

$$
\delta W=\delta W_{u}-\delta W_{\phi},
$$

with its terms given by

$$
\begin{aligned}
\delta W_{u} & =\int_{\Omega_{0}} Z \delta w_{0} d \alpha d \beta+\oint_{\Gamma_{\alpha}}\left[\int_{-h}^{+h}\left(\hat{\sigma}_{\beta \beta} \delta v+\hat{\sigma}_{\beta \alpha} \delta u+\hat{\sigma}_{\beta z} \delta w\right) \frac{1}{z_{\alpha}^{(0)}} d z\right] d \alpha \\
& +\oint_{\Gamma_{\beta}}\left[\int_{-h}^{+h}\left(\hat{\sigma}_{\alpha \alpha} \delta u+\hat{\sigma}_{\alpha \beta} \delta v+\hat{\sigma}_{z \alpha} \delta w\right) \frac{1}{z_{\beta}^{(0)}} d z\right] d \beta, \\
\delta W_{\phi} & =\int_{\Omega_{0}} \tau \delta \phi d \alpha d \beta,
\end{aligned}
$$

where $Z=F_{z}^{t}\left(1+h / R_{\alpha}\right)\left(1+h / R_{\beta}\right)$ and $\tau=\tau^{t} \lambda\left(1+h / R_{\alpha}\right)\left(1+h / R_{\beta}\right)$. The virtual work of the nonconservative forces $\delta W_{u}$ is expressed with more detail in terms of the variations of the generalized displacements and prescribed forces and moments in Appendix F3. 


\section{E. Weak Forms of the Governing Equations in Terms of Internal Forces and Moments}

The weak form of the governing electro-mechanical equations in terms of internal forces and moments are obtained by substituting the variational terms in Eqs. (52), (54) and (58) into the Hamilton's principle in Eq. (50). The virtual generalized displacements, surface stresses and electric potential difference are zero where the corresponding variables are specified. For time-dependent problems, the admissible virtual generalized variables must also vanish at time $t=t_{0}$ and $t=t_{1}$. Thus, using the fundamental lema of variational calculus and collecting the coefficients of each variation of the different generalized variables (displacements, surface stresses and electric potential) into independent equations yields for the generalized displacements $\delta u_{0}, \delta v_{0}, \delta w_{0}, \delta \theta_{\alpha}$ and $\delta \theta_{\beta}$ :

$$
\begin{aligned}
& \int_{\Omega_{0}}\left[\delta u_{0}\left(I_{11}^{u u} \ddot{u}_{0}+I_{13}^{u u} \frac{\partial \ddot{w}_{0}}{\partial \alpha}+I_{14}^{u u} \ddot{\theta}_{\alpha}+I_{11}^{\tau u} \ddot{\bar{\tau}}_{z \alpha}+I_{21}^{\tau u} \ddot{\tilde{\tau}}_{z \alpha}\right)+\delta \frac{\partial u_{0}}{\partial \alpha}\left(N_{\alpha \alpha}^{\star 11}+N_{\beta \beta}^{\star 21}\right)\right. \\
& \left.+\delta \frac{\partial u_{0}}{\partial \beta}\left(N_{\alpha \beta}^{51}\right)+\delta u_{0}\left(Q_{z \alpha}^{41}\right)\right] d \alpha d \beta-\oint_{\Gamma_{\alpha}} \delta u_{0}\left(\hat{N}_{\alpha \beta}^{11}\right) d \alpha-\oint_{\Gamma_{\beta}} \delta u_{0}\left(\hat{N}_{\alpha \alpha}^{11}\right) d \beta=0, \\
& \int_{\Omega_{0}}\left[\delta v_{0}\left(I_{22}^{u u} \ddot{v}_{0}+I_{23}^{u u} \frac{\partial \ddot{w}_{0}}{\partial \beta}+I_{25}^{u u} \ddot{\theta}_{\beta}+I_{32}^{\tau u} \ddot{\bar{\tau}}_{\beta z}+I_{42}^{\tau u} \ddot{\tilde{\tau}}_{\beta z}\right)+\delta \frac{\partial v_{0}}{\partial \beta}\left(N_{\alpha \alpha}^{\star 12}+N_{\beta \beta}^{\star 22}\right)\right. \\
& \left.+\delta \frac{\partial v_{0}}{\partial \alpha}\left(N_{\alpha \beta}^{52}\right)+\delta v_{0}\left(Q_{\beta z}^{32}\right)\right] d \alpha d \beta-\oint_{\Gamma_{\alpha}} \delta v_{0}\left(\hat{N}_{\beta \beta}^{22}\right) d \alpha-\oint_{\Gamma_{\beta}} \delta v_{0}\left(\hat{N}_{\alpha \beta}^{22}\right) d \beta=0, \\
& \int_{\Omega_{0}}\left[\delta w_{0}\left(I_{33}^{u u} \ddot{w}\right)+\delta \frac{\partial w_{0}}{\partial \alpha}\left(I_{13}^{u u} \ddot{u}_{0}+I_{33}^{u \alpha} \frac{\partial \ddot{w}_{0}}{\partial \alpha}+I_{34}^{u u} \ddot{\theta}_{\alpha}+I_{13}^{\tau u} \ddot{\bar{\tau}}_{z \alpha}+I_{23}^{\tau u} \ddot{\tilde{\tau}}_{z \alpha}\right)+\delta \frac{\partial w_{0}}{\partial \beta}\left(I_{23}^{u u} \ddot{v}_{0}+I_{33}^{u \beta} \frac{\partial \ddot{w}_{0}}{\partial \beta}\right.\right. \\
& \left.+I_{35}^{u u} \ddot{\theta}_{\beta}+I_{33}^{\tau u} \ddot{\bar{\tau}}_{\beta z}+I_{43}^{\tau u} \ddot{\tilde{\tau}}_{\beta z}\right)+\delta w_{0}\left(M_{\alpha \alpha}^{\star 131}+M_{\beta \beta}^{\star 231}\right)+\delta \frac{\partial^{2} w_{0}}{\partial \alpha^{2}}\left(M_{\alpha \alpha}^{\star 132}\right)+\delta \frac{\partial^{2} w_{0}}{\partial \beta^{2}}\left(M_{\beta \beta}^{\star 232}\right) \\
& \left.+\delta \frac{\partial^{2} w_{0}}{\partial \alpha \partial \beta}\left(M_{\alpha \beta}^{53}\right)+\delta \frac{\partial w_{0}}{\partial \alpha}\left(Q_{z \alpha}^{43}\right)+\delta \frac{\partial w_{0}}{\partial \beta}\left(Q_{\beta z}^{33}\right)-\delta w_{0} Z\right] d \alpha d \beta-\oint_{\Gamma_{\alpha}}\left[\delta \frac{\partial w_{0}}{\partial \beta}\left(\hat{M}_{\beta \beta}^{23}\right)\right. \\
& \left.+\delta \frac{\partial w_{0}}{\partial \alpha}\left(\hat{M}_{\alpha \beta}^{13}\right)+\delta w_{0}\left(\hat{Q}_{\beta z}^{33}\right)\right] d \alpha-\oint_{\Gamma_{\beta}}\left[\delta \frac{\partial w_{0}}{\partial \alpha}\left(\hat{M}_{\alpha \alpha}^{13}\right)+\delta \frac{\partial w_{0}}{\partial \beta}\left(\hat{M}_{\alpha \alpha}^{23}\right)+\delta w_{0}\left(\hat{Q}_{z \alpha}^{33}\right)\right] d \beta=0, \\
& \int_{\Omega_{0}}\left[\delta \theta_{0}^{\alpha}\left(I_{14}^{u u} \ddot{u}_{0}+I_{43}^{u u} \frac{\partial \ddot{w}_{0}}{\partial \alpha}+I_{44}^{u u} \ddot{\theta}_{0}^{\alpha}+I_{14}^{\tau u} \ddot{\tilde{\tau}}_{z \alpha}+I_{24}^{\tau u} \ddot{\tilde{\tau}}_{z \alpha}\right)+\delta \frac{\partial \theta_{\alpha}}{\partial \alpha}\left(M_{\alpha \alpha}^{\star 14}+M_{\beta \beta}^{\star 24}\right)\right. \\
& \left.+\delta \frac{\partial \theta_{\alpha}}{\partial \beta}\left(M_{\alpha \beta}^{54}\right)+\delta \theta_{\alpha}\left(Q_{z \alpha}^{44}\right)\right] d \alpha d \beta-\oint_{\Gamma_{\alpha}} \delta \theta_{\alpha}\left(\hat{M}_{\alpha \beta}^{14}\right) d \alpha-\oint_{\Gamma_{\beta}} \delta \theta_{\alpha}\left(\hat{M}_{\alpha \alpha}^{14}\right) d \beta=0, \\
& \int_{\Omega_{0}}\left[\delta \theta_{0}^{\beta}\left(I_{25}^{u u} \ddot{v}_{0}+I_{35}^{u u} \frac{\partial \ddot{w}_{0}}{\partial \beta}+I_{55}^{u u} \ddot{\theta}_{0}^{\beta}+I_{35}^{\tau u} \ddot{\bar{\tau}}_{\beta z}+I_{45}^{\tau u} \ddot{\tilde{\tau}}_{\beta z}\right)+\delta \frac{\partial \theta_{\beta}}{\partial \beta}\left(M_{\alpha \alpha}^{\star 15}+M_{\beta \beta}^{\star 25}\right)\right. \\
& \left.+\delta \frac{\partial \theta_{\beta}}{\partial \alpha}\left(M_{\alpha \beta}^{55}\right)+\delta \theta_{\beta}\left(Q_{\beta z}^{35}\right)\right] d \alpha d \beta-\oint_{\Gamma_{\alpha}} \delta \theta_{\beta}\left(\hat{M}_{\beta \beta}^{25}\right) d \alpha-\oint_{\Gamma_{\beta}} \delta \theta_{\beta}\left(\hat{M}_{\alpha \beta}^{25}\right) d \beta=0 .
\end{aligned}
$$

In a similar way, for the generalized surface stress variables $\delta \bar{\tau}_{z \alpha}, \delta \tilde{\tau}_{z \alpha}, \delta \bar{\tau}_{\beta z}$ and $\delta \tilde{\tau}_{\beta z}$ one gets

$$
\begin{aligned}
& \int_{\Omega_{0}}\left[\delta \bar{\tau}_{z \alpha}\left(I_{11}^{\tau u} \ddot{u}_{0}+I_{13}^{\tau u} \frac{\partial \ddot{w}_{0}}{\partial \alpha}+I_{14}^{\tau u} \ddot{\theta}_{\alpha}+I_{11}^{\tau \tau} \ddot{\bar{\tau}}_{z \alpha}+I_{12}^{\tau \tau} \ddot{\tilde{\tau}}_{z \alpha}\right)+\delta \frac{\partial \bar{\tau}_{z \alpha}}{\partial \alpha}\left(T_{\alpha \alpha}^{\star 11}+T_{\beta \beta}^{\star 21}\right)\right. \\
& \left.+\delta \bar{\tau}_{z \alpha}\left(T_{z \alpha}^{41}\right)+\delta \frac{\partial \bar{\tau}_{z \alpha}}{\partial \beta}\left(T_{\alpha \beta}^{51}\right)\right] d \alpha d \beta-\oint_{\Gamma_{\alpha}} \delta \bar{\tau}_{z \alpha}\left(\hat{T}_{\beta \alpha}^{11}\right) d \alpha-\oint_{\Gamma_{\beta}} \delta \bar{\tau}_{z \alpha}\left(\hat{T}_{\alpha \alpha}^{11}\right) d \beta=0, \\
& \int_{\Omega_{0}}\left[\delta \tilde{\tau}_{z \alpha}\left(I_{21}^{\tau u} \ddot{u}_{0}+I_{23}^{\tau u} \frac{\partial \ddot{w}_{0}}{\partial \alpha}+I_{24}^{\tau u} \ddot{\theta}_{\alpha}+I_{12}^{\tau \tau} \ddot{\bar{\tau}}_{z \alpha}+I_{22}^{\tau \tau} \ddot{\tilde{\tau}}_{z \alpha}\right)+\delta \frac{\partial \tilde{\tau}_{z \alpha}}{\partial \alpha}\left(T_{\alpha \alpha}^{\star 12}+T_{\beta \beta}^{\star 22}\right)\right. \\
& \left.+\delta \tilde{\tau}_{z \alpha}\left(T_{z \alpha}^{42}\right)+\delta \frac{\partial \tilde{\tau}_{z \alpha}}{\partial \beta}\left(T_{\alpha \beta}^{52}\right)\right] d \alpha d \beta-\oint_{\Gamma_{\alpha}} \delta \tilde{\tau}_{z \alpha}\left(\hat{T}_{\beta \alpha}^{12}\right) d \alpha-\oint_{\Gamma_{\beta}} \delta \tilde{\tau}_{z \alpha}\left(\hat{T}_{\alpha \alpha}^{12}\right) d \beta=0,
\end{aligned}
$$




$$
\begin{aligned}
& \int_{\Omega_{0}}\left[\delta \bar{\tau}_{\beta z}\left(I_{32}^{\tau u} \ddot{v}_{0}+I_{33}^{\tau u} \frac{\partial \ddot{w}_{0}}{\partial \beta}+I_{35}^{\tau u} \ddot{\theta}_{\beta}+I_{33}^{\tau \tau} \ddot{\bar{\tau}}_{\beta z}+I_{34}^{\tau \tau} \ddot{\tilde{\tau}}_{\beta z}\right)+\delta \frac{\partial \bar{\tau}_{\beta z}}{\partial \beta}\left(T_{\alpha \alpha}^{\star 13}+T_{\beta \beta}^{\star 23}\right)\right. \\
& \left.+\delta \bar{\tau}_{\beta z}\left(T_{\beta z}^{33}\right)+\delta \frac{\partial \bar{\tau}_{\beta z}}{\partial \alpha}\left(T_{\alpha \beta}^{53}\right)\right] d \alpha d \beta-\oint_{\Gamma_{\alpha}} \delta \bar{\tau}_{\beta z}\left(\hat{T}_{\beta \beta}^{23}\right) d \alpha-\oint_{\Gamma_{\beta}} \delta \bar{\tau}_{\beta z}\left(\hat{T}_{\alpha \beta}^{23}\right) d \beta=0, \\
& \int_{\Omega_{0}}\left[\delta \tilde{\tau}_{\beta z}\left(I_{42}^{\tau u} \ddot{v}_{0}+I_{43}^{\tau u} \frac{\partial \ddot{w}_{0}}{\partial \beta}+I_{45}^{\tau u} \ddot{\theta}_{\beta}+I_{34}^{\tau \tau} \ddot{\bar{\tau}}_{\beta z}+I_{44}^{\tau \tau} \ddot{\tilde{\tau}}_{z \alpha}\right)+\delta \frac{\partial \tilde{\tau}_{\beta z}}{\partial \beta}\left(T_{\alpha \alpha}^{\star 14}+T_{\beta \beta}^{\star 24}\right)\right. \\
& \left.+\delta \tilde{\tau}_{\beta z}\left(T_{\beta z}^{34}\right)+\delta \frac{\partial \tilde{\tau}_{\beta z}}{\partial \alpha}\left(T_{\alpha \beta}^{54}\right)\right] d \alpha d \beta-\oint_{\Gamma_{\alpha}} \delta \tilde{\tau}_{\beta z}\left(\hat{T}_{\beta \beta}^{24}\right) d \alpha-\oint_{\Gamma_{\beta}} \delta \tilde{\tau}_{\beta z}\left(\hat{T}_{\alpha \beta}^{24}\right) d \beta=0 .
\end{aligned}
$$

The last equation regards the electrostatic equilibrium and is obtained by collecting the coefficients of the variation of the generalized electric potential difference $\delta \phi$ as

$$
\begin{gathered}
\int_{\Omega_{0}}\left[\delta \phi \left[\left(P_{\alpha \alpha}^{\star 11}+P_{\beta \beta}^{\star 21}\right) \frac{\partial u_{0}}{\partial \alpha}+\left(P_{\alpha \alpha}^{\star 12}+P_{\beta \beta}^{\star 22}\right) \frac{\partial v_{0}}{\partial \beta}+\left(P_{\alpha \alpha 1}^{\star 13}+P_{\alpha \alpha 1}^{\star 23}\right) w_{0}+\left(P_{\alpha \alpha 2}^{\star 13}\right) \frac{\partial^{2} w_{0}}{\partial \alpha^{2}}+\left(P_{\beta \beta 2}^{\star 23}\right) \frac{\partial^{2} w_{0}}{\partial \beta^{2}}\right.\right. \\
+\left(P_{\alpha \alpha}^{\star 14}+P_{\beta \beta}^{\star 24}\right) \frac{\partial \theta_{\alpha}}{\partial \alpha}+\left(P_{\alpha \alpha}^{\star 15}+P_{\beta \beta}^{\star 25}\right) \frac{\partial \theta_{\beta}}{\partial \beta}+\left(R_{\alpha \alpha}^{\star 11}+R_{\beta \beta}^{\star 21}\right) \frac{\partial \bar{\tau}_{z \alpha}}{\partial \alpha}+\left(R_{\alpha \alpha}^{\star 12}+R_{\beta \beta}^{\star 22}\right) \frac{\partial \tilde{\tau}_{z \alpha}}{\partial \alpha} \\
\left.+\left(R_{\alpha \alpha}^{\star 13}+R_{\beta \beta}^{\star 23}\right) \frac{\partial \bar{\tau}_{\beta z}}{\partial \beta}+\left(R_{\alpha \alpha}^{\star 14}+R_{\beta \beta}^{\star 24}\right) \frac{\partial \tilde{\tau}_{\beta z}}{\partial \beta}+\left(S_{\phi \phi}\right) \phi-\tau\right] d \alpha d \beta=0 .
\end{gathered}
$$

The previous equations are the weak forms of the governing equations of the doubly-curved orthotropic generic piezoelectric shell layer. As can be seen, the 3-D problem has been brought to a 2-D form in function of the reference surface curvilinear coordinates $\alpha$ and $\beta$. Hence, the FE solution of the shell problem can be derived in a manner similar to that of plates with some additional terms regarding the curvatures. It is worthy to mention that in the present refined shell theory no assumptions regarding the thinness of the shell were considered and as a consequence the formulation fully accounts for the effects of the $z / R_{\alpha}$ and $z / R_{\beta}$ terms. Additionally, the "mixed" partially refined theory also considers additional generalized variables concerning the shear stresses on the top and bottom surfaces of the shell layer which will be used at the elemental FE level to impose transverse interlaminar (interlayer) continuity of the shear stresses and homogeneous shear stress conditions on the top and bottom global surfaces of the multilayer shell.

\section{F. Constitutive Equations of the Internal Forces and Moments}

The strains, and there by the stresses, of the proposed theory where shown to be non-linearly dependent across the thickness of a thick anisotropic elastic shell. Thus, as far as the mathematical model is concerned, it is convenient to integrate the stress distributions through the thickness of the shell and to replace the usual consideration of stress by statically equivalent internal forces and moments. By performing such integration, the variations with respect to the thickness coordinate $z$ are completely eliminated to yield a 2-D mathematical model of the 3-D physical problem. These integrations were carried out in Appendix F, and the virtual work quantities of Hamilton's principle in Eq. (50) were expressed in terms of internal forces and moments.

Contrarily to what is often presented in the literature, and since the thickness terms $z / R_{\alpha}$ and $z / R_{\beta}$ were fully retained in the formulation, in the definitions of force and moment resultants given in Eqs. (F5) and (F9) of Appendix F one may notice that the symmetry of the stress tensor (that is, $\sigma_{\alpha \beta}=\sigma_{\beta \alpha}$ ) doesn't necessarily implies that the correspondent force resultants or the moment resultants are equal, even if we consider the restriction of dealing with a doubly-curved shell as stated in Eqs. (39). That relation holds only for a spherical shell, flat plate or a thin shell of any type where the assumptions $1+z / R_{\alpha} \approx 1$ and $1+z / R_{\beta} \approx 1$ are taken into account. Vanishing of the moments about the normal to the differential element yields an additional relation among the twisting moments and twisting shear forces (cf. [6, Sec. 8.2.4]). In order to avoid inconsistency associated with rigid body rotations (i.e., rigid body rotation gives a nonvanishing torsion except for flat plates or spherical shells) the additional relation must be accounted for in the formulation (see the treatment of Sanders which is described for example by Kraus [1, Sec. 3.2], Leissa [4, Sec. 1.4.5] or Reddy [6, Sec. 8.2.4]). However, if the rotation is of the same order of magnitude as the strain components, which is actually the case in most problems, then, as noted by Koiter, ${ }^{7}$ the torsion is negligible. Thus, for general engineering purposes the foregoing inconsistencies can generally be overlooked which will be the case in this work. 
Next the constitutive equations that relate the internal forces and moments in Eqs. (F5) of Appendix with the strains of the layer and/or generalized displacements are derived. To this end it is recalled that an orthotropic piezoelectric material is considered for the generic shell layer and that it obeys Hooke's law under plane-stress assumption as defined in Eq. (C15) of Appendix. Thus, the internal in-plane forces are collected as

$$
\left\{\begin{array}{c}
\left(N_{\alpha \alpha}^{\star 11}, N_{\alpha \alpha}^{\star 12}\right) \\
\left(N_{\beta \beta}^{\star 21}, N_{\beta \beta}^{\star 22}\right) \\
\left(N_{\alpha \beta}^{51}, N_{\alpha \beta}^{52}\right)
\end{array}\right\}=\left\langle\left\{\begin{array}{c}
\sigma_{\alpha \alpha}\left(z_{11}^{\star \varepsilon u}, z_{12}^{\star \varepsilon u}\right) \\
\sigma_{\beta \beta}\left(z_{21}^{\star \varepsilon u}, z_{22}^{\star \varepsilon u}\right) \\
\sigma_{\alpha \beta}\left(z_{51}^{\varepsilon u}, z_{52}^{\varepsilon u}\right)
\end{array}\right\}\right\rangle-\left\langle\left\{\begin{array}{c}
\sigma_{\alpha \alpha}\left(z_{11}^{\star \varepsilon u}, z_{12}^{\star \varepsilon u}\right) \\
\sigma_{\beta \beta}\left(z_{21}^{\star \varepsilon u}, z_{22}^{\star \varepsilon u}\right) \\
\sigma_{\alpha \beta}\left(z_{51}^{\varepsilon u}, z_{52}^{\varepsilon u}\right)
\end{array}\right\}\right\rangle
$$

where the superscripts $(\cdot)^{u}$ and $(\cdot)^{\phi}$ denote quantities referred to the mechanical displacements (or strains) and electrical potential, and for convenience $\langle\ldots\rangle$ denotes thickness integration defined as

$$
\langle\ldots\rangle=\int_{-h}^{+h}(\ldots) \frac{1}{z_{\alpha}^{(0)} z_{\beta}^{(0)}} d z
$$

Therefore, considering the converse piezoelectric constitutive behavior in Eq. (C15) and considering only the prescribed electric field component $\hat{E}_{z}$ yields

$$
\left\{\begin{array}{c}
\left(N_{\alpha \alpha}^{\star 11}, N_{\alpha \alpha}^{\star 12}\right) \\
\left(N_{\beta \beta}^{\star 21}, N_{\beta \beta}^{\star 22}\right) \\
\left(N_{\alpha \beta}^{51}, N_{\alpha \beta}^{52}\right)
\end{array}\right\}=\left\langle\left[\begin{array}{ccc}
\bar{c}_{11}^{*} & \bar{c}_{12}^{*} & 0 \\
\bar{c}_{12}^{*} & \bar{c}_{22}^{*} & 0 \\
0 & 0 & \bar{c}_{66}^{*}
\end{array}\right]\left\{\begin{array}{c}
\varepsilon_{\alpha \alpha}\left(z_{11}^{\star \varepsilon u}, z_{12}^{\star \varepsilon u}\right) \\
\varepsilon_{\beta \beta}\left(z_{21}^{\star \varepsilon u}, z_{22}^{\star \varepsilon u}\right) \\
\varepsilon_{\alpha \beta}\left(z_{51}^{\varepsilon u}, z_{52}^{\varepsilon u}\right)
\end{array}\right\}\right\rangle-\left\langle\left\{\begin{array}{c}
\bar{e}_{31}\left(z_{11}^{\star \varepsilon u}, z_{12}^{\star \varepsilon u}\right) \\
\bar{e}_{32}\left(z_{21}^{\star \varepsilon u}, z_{22}^{\star \varepsilon u}\right) \\
0
\end{array}\right\} \hat{E}_{z}\right\rangle .
$$

Similarly, the out-of-plane forces are collected as

$$
\left\{\begin{array}{l}
\left(Q_{\beta z}^{32}, Q_{\beta z}^{33}, Q_{\beta z}^{35}\right) \\
\left(Q_{z \alpha}^{41}, Q_{z \alpha}^{43}, Q_{z \alpha}^{44}\right)
\end{array}\right\}=\left\langle\left\{\begin{array}{l}
\sigma_{\beta z}\left(z_{32}^{\varepsilon u}, z_{33}^{\varepsilon u}, z_{35}^{\varepsilon u}\right) \\
\sigma_{z \alpha}\left(z_{41}^{\varepsilon u}, z_{43}^{\varepsilon u}, z_{44}^{\varepsilon u}\right)
\end{array}\right\}\right\rangle
$$

which, since they are not coupled with the transverse electric field, but only with the induced in-plain components which effects were already considered through the use of effective shear stiffness parameters, is expressed in terms of the shear strains as

$$
\left\{\begin{array}{l}
\left(Q_{\beta z}^{32}, Q_{\beta z}^{33}, Q_{\beta z}^{35}\right) \\
\left(Q_{z \alpha}^{41}, Q_{z \alpha}^{43}, Q_{z \alpha}^{44}\right)
\end{array}\right\}=\left\langle\left[\begin{array}{cc}
\bar{c}_{44}^{\star} & 0 \\
0 & \bar{c}_{55}^{\star}
\end{array}\right]\left\{\begin{array}{l}
\varepsilon_{\beta z}\left(z_{32}^{\varepsilon u}, z_{33}^{\varepsilon u}, z_{35}^{\varepsilon u}\right) \\
\varepsilon_{z \alpha}\left(z_{41}^{\varepsilon u}, z_{43}^{\varepsilon u}, z_{44}^{\varepsilon u}\right)
\end{array}\right\}\right\rangle .
$$

The moment resultants of the in-plane stresses are collected and expressed by

$$
\begin{aligned}
\left\{\begin{array}{c}
\left(M_{\alpha \alpha}^{\star 131}, M_{\alpha \alpha}^{\star 132}, M_{\alpha \alpha}^{\star 14}, M_{\alpha \alpha}^{\star 15}\right) \\
\left(M_{\beta \beta}^{\star 231}, M_{\beta \beta}^{\star 232}, M_{\beta \beta}^{\star 24}, M_{\beta \beta}^{\star 25}\right) \\
\left(M_{\alpha \beta}^{53}, M_{\alpha \beta}^{54}, M_{\alpha \beta}^{55}\right)
\end{array}\right\} & =\left\langle\left\{\begin{array}{c}
\sigma_{\alpha \alpha}\left(z_{13}^{\star \varepsilon u 1}, z_{13}^{\star \varepsilon u 2}, z_{14}^{\star \varepsilon u}, z_{15}^{\star \varepsilon u}\right) \\
\left.\sigma_{\beta \beta}\left(z_{23}^{\star \varepsilon u 1}, z_{23}^{\star \varepsilon u 2}, z_{24}^{\star \varepsilon u}, z_{25}^{\star \varepsilon u}\right)\right\} \\
\sigma_{\alpha \beta}\left(z_{53}^{\varepsilon u}, z_{54}^{\varepsilon u}, z_{55}^{\varepsilon u}\right)
\end{array}\right\}\right. \\
& -\left\langle\left\{\begin{array}{c}
\sigma_{\alpha \alpha}\left(z_{13}^{\star \varepsilon u 1}, z_{13}^{\star \varepsilon u 2}, z_{14}^{\star \varepsilon u}, z_{15}^{\star \varepsilon u}\right) \\
\sigma_{\beta \beta}\left(z_{23}^{\star \varepsilon u 1}, z_{23}^{\star \varepsilon u 2}, z_{24}^{\star \varepsilon u}, z_{25}^{\star \varepsilon u}\right) \\
\sigma_{\alpha \beta}\left(z_{53}^{\varepsilon u}, z_{54}^{\varepsilon u}, z_{55}^{\varepsilon u}\right)
\end{array}\right\} .\right.
\end{aligned}
$$

Similarly to what has been considered for the in-plane forces in Eq. (73) the internal moments are re-written as

$$
\begin{aligned}
\left\{\begin{array}{c}
\left(M_{\alpha \alpha}^{\star 131}, M_{\alpha \alpha}^{\star 132}, M_{\alpha \alpha}^{\star 14}, M_{\alpha \alpha}^{\star 15}\right) \\
\left(M_{\beta \beta}^{\star 231}, M_{\beta \beta}^{\star 232}, M_{\beta \beta}^{\star 24}, M_{\beta \beta}^{\star 25}\right) \\
\left(M_{\alpha \beta}^{53}, M_{\alpha \beta}^{54}, M_{\alpha \beta}^{55}\right)
\end{array}\right\} & =\left\langle\left[\begin{array}{ccc}
\bar{c}_{11}^{*} & \bar{c}_{12}^{*} & 0 \\
\bar{c}_{12}^{*} & \bar{c}_{22}^{*} & 0 \\
0 & 0 & \bar{c}_{66}^{*}
\end{array}\right]\left\{\begin{array}{c}
\varepsilon_{\alpha \alpha}\left(z_{13}^{\star \varepsilon u 1}, z_{13}^{\star \varepsilon u 2}, z_{14}^{\star \varepsilon u}, z_{15}^{\star \varepsilon u}\right) \\
\varepsilon_{\beta \beta}\left(z_{23}^{\star \varepsilon u 1}, z_{23}^{\star \varepsilon u 2}, z_{24}^{\star \varepsilon u}, z_{25}^{\star \varepsilon u}\right) \\
\varepsilon_{\alpha \beta}\left(z_{53}^{\varepsilon u}, z_{54}^{\varepsilon u}, z_{55}^{\varepsilon u}\right)
\end{array}\right\}\right\rangle \\
& -\left\langle\left\{\begin{array}{c}
\bar{e}_{31}\left(z_{13}^{\star \varepsilon u 1}, z_{13}^{\star \varepsilon u 2}, z_{14}^{\star \varepsilon u}, z_{15}^{\star \varepsilon u}\right) \\
\bar{e}_{32}\left(z_{23}^{\star \varepsilon u 1}, z_{23}^{\star \varepsilon u 2}, z_{24}^{\star \varepsilon u}, z_{25}^{\star \varepsilon u}\right) \\
0
\end{array}\right\} \hat{E}_{z}\right\rangle .
\end{aligned}
$$


By last, in a similar way to what has been done in Eqs. (73) and (75), the resultants of the stresses related with the interlayer surface stresses are expressed by

$$
\begin{aligned}
\left\{\begin{array}{c}
\left(T_{\alpha \alpha}^{\star 11}, T_{\alpha \alpha}^{\star 12}, T_{\alpha \alpha}^{\star 13}, T_{\alpha \alpha}^{\star 14}\right) \\
\left(T_{\beta \beta}^{\star 21}, T_{\beta \beta}^{\star 22}, T_{\beta \beta}^{\star 23}, T_{\beta \beta}^{\star 24}\right) \\
\left(T_{\alpha \beta}^{51}, T_{\alpha \beta}^{52}, T_{\alpha \beta}^{53}, T_{\alpha \beta}^{54}\right)
\end{array}\right\} & =\left\langle\left[\begin{array}{ccc}
\bar{c}_{11}^{*} & \bar{c}_{12}^{*} & 0 \\
\bar{c}_{12}^{*} & \bar{c}_{22}^{*} & 0 \\
0 & 0 & \bar{c}_{66}^{*}
\end{array}\right]\left\{\begin{array}{c}
\varepsilon_{\alpha \alpha}\left(z_{11}^{\star \varepsilon \tau}, z_{12}^{\star \varepsilon \tau}, z_{13}^{\star \varepsilon \tau}, z_{14}^{\star \varepsilon \tau}\right) \\
\varepsilon_{\beta \beta}\left(z_{21}^{\star \varepsilon \tau}, z_{22}^{\star \varepsilon \tau}, z_{23}^{\star \varepsilon \tau}, z_{24}^{\star \varepsilon \tau}\right) \\
\varepsilon_{\alpha \beta}\left(z_{51}^{\varepsilon \tau}, z_{52}^{\varepsilon \tau}, z_{53}^{\varepsilon \tau}, z_{54}^{\varepsilon \tau}\right)
\end{array}\right\}\right\rangle \\
& -\left\langle\left\{\begin{array}{c}
\bar{e}_{31}\left(z_{11}^{\star \varepsilon \tau}, z_{12}^{\star \varepsilon \tau}, z_{13}^{\star \varepsilon \tau}, z_{14}^{\star \varepsilon \tau}\right) \\
\bar{e}_{32}\left(z_{21}^{\star \varepsilon \tau}, z_{22}^{\star \varepsilon \tau}, z_{23}^{\star \varepsilon \tau}, z_{24}^{\star \varepsilon \tau}\right) \\
0
\end{array}\right\} \hat{E}_{z}\right\rangle,
\end{aligned}
$$

and

$$
\left\{\begin{array}{l}
\left(T_{\beta z}^{33}, T_{\beta z}^{34}\right) \\
\left(T_{z \alpha}^{41}, T_{z \alpha}^{42}\right)
\end{array}\right\}=\left\langle\left[\begin{array}{cc}
\bar{c}_{44}^{\star} & 0 \\
0 & \bar{c}_{55}^{\star}
\end{array}\right]\left\{\begin{array}{l}
\varepsilon_{\beta z}\left(z_{33}^{\varepsilon \tau}, z_{34}^{\varepsilon \tau}\right) \\
\varepsilon_{z \alpha}\left(z_{41}^{\varepsilon \tau}, z_{42}^{\varepsilon \tau}\right)
\end{array}\right\}\right\rangle .
$$

For the sake of simplicity of exposition of the internal forces and moments, the previous equations are not developed here in terms of the generalized displacements and electric potential. The reader is referred to Appendix G to further details concerning that matter.

\section{Finite Element Solution}

\section{A. Preliminary Comments on the FE Solution of the Fully and Partially Refined Models}

In this section the FE solution of the weak form of the governing electro-mechanical coupled equations of the partially refined mathematical model of doubly curved shells in Eqs. (61)-(70) is developed. Regarding the FE solution of the fully refined model, it will not be derived here for reasons related with the complexity of the formulation. As can be seen from the fully refined definitions of the displacement field presented in Eqs. (20), (23) and (24), the fully refined weak forms would involve high-order derivatives of the generalized displacements which would complicate the formulation and FE solution dramatically. That would require higher order continuity of the variables, which would be cumbersome for FE solutions, with the outcome of considering an equivalent 2-D theory representative of the full 3-D elasticity problem. It is well known, however, that in the major part of the problems, the transverse stress is small when compared with the other stress components. An exception is, for example, in thermo-mechanical analysis where the transverse stress $\sigma_{z z}$ plays an important role (see Carrera ${ }^{8,106}$ ), which is not the case here. That refinement is not pursued here since the trade-off between accuracy and complexity is not appellative for the physical problem to be treated in this work.

\section{B. Spatial Approximation}

For the sake of brevity the weak forms of the partially refined model in Eqs. (61)-(70) are expressed in terms of the internal forces and moments (stress resultants). However, if the constitutive equations of the internal forces and moments, presented in Sec. F and detailed in Appendix G, are taken into account, the weak forms can still be expressed in terms of the generalized variables. That will not be made here explicitly, but those relations will be implicitly taken into account to derive the elemental matrices and vectors.

Thus, from the analysis of the weak forms in (61)-(70) and/or the internal forces and moments in Appendix $\mathrm{G}$, it can be seen that they contain at the most first-order derivatives of the generalized displacements $u_{0}, v_{0}, \theta_{\alpha}$ and $\theta_{\beta}$ and surface stresses $\bar{\tau}_{z \alpha}, \tilde{\tau}_{z \alpha}, \bar{\tau}_{\beta z}$ and $\tilde{\tau}_{\beta z}$, requiring $C^{0}$ continuity, and in contrast to what is traditionally obtained with the FSDT, the present partial refined model contain also at the most second-order derivatives of the transverse displacement $w_{0}$, requiring $C^{1}$ continuity, which is something that is obtained with the CLT. Thus, the partially refined model, at first sight, yields something that resembles a blend of the CLT and FSDT. Additionally, the electric potential difference requires zero-order derivatives, and is assumed constant at the elemental electrodes, which is convenient since it imposes at least at the elemental level the physical equipotential area condition of the electrodes, which should also be assumed between adjacent FEs. Thus, the displacement variables, $u_{0}, v_{0}, \theta_{\alpha}, \theta_{\beta}, w_{0}, \partial w_{0} / \partial \alpha, \partial w_{0} / \partial \beta$ and (or not) $\partial^{2} w_{0} / \partial \alpha \partial \beta$ (nonconforming or conforming elemental approaches), and shear stress variables, $\bar{\tau}_{z \alpha}, \tilde{\tau}_{z \alpha}, \bar{\tau}_{\beta z}$ and $\tilde{\tau}_{\beta z}$, must be carried as nodal variables in order to enforce their interelement continuity. Regarding the 
electric potential difference variable, $\phi$, is carried out as an elemental variable and interelement continuity is not enforced.

For the $\mathrm{FE}$ solution, linear Lagrange $C^{0}$ continuity rectangular interpolation functions might be used to approximate all the displacement and stress variables whereas the generalized transverse displacement $w_{0}$ should be approximated using Hermite $C^{1}$ continuity rectangular interpolation functions over a four-noded element $\Omega_{0}^{e}$. The combined conforming or nonconforming elements have a total of 12 or 11 degrees of freedom (DoFs) per node, respectively, and 1 electrical DoF per element. Therefore, let

$$
\begin{aligned}
u_{0}(\alpha, \beta, t) & \approx \sum_{j=1}^{n} \bar{u}_{0}^{j}(t) L_{j}^{e}(\alpha, \beta), & v_{0}(\alpha, \beta, t) & \approx \sum_{j=1}^{n} \bar{v}_{0}^{j}(t) L_{j}^{e}(\alpha, \beta), \\
\theta_{\alpha}(\alpha, \beta, t) & \approx \sum_{j=1}^{n} \bar{\theta}_{\alpha}^{j}(t) L_{j}^{e}(\alpha, \beta), & \theta_{\beta}(\alpha, \beta, t) & \approx \sum_{j=1}^{n} \bar{\theta}_{\beta}^{j}(t) L_{j}^{e}(\alpha, \beta), \\
\bar{\tau}_{z \alpha}(\alpha, \beta, t) & \approx \sum_{j=1}^{n} \overline{\bar{\tau}}_{z \alpha}^{j}(t) L_{j}^{e}(\alpha, \beta), & \tilde{\tau}_{z \alpha}(\alpha, \beta, t) & \approx \sum_{j=1}^{n} \overline{\tilde{\tau}}_{z \alpha}^{j}(t) L_{j}^{e}(\alpha, \beta), \\
\bar{\tau}_{\beta z}(\alpha, \beta, t) & \approx \sum_{j=1}^{n} \overline{\bar{\tau}}_{\beta z}^{j}(t) L_{j}^{e}(\alpha, \beta), & \tilde{\tau}_{\beta z}(\alpha, \beta, t) & \approx \sum_{j=1}^{n} \overline{\tilde{\tau}}_{\beta z}^{j}(t) L_{j}^{e}(\alpha, \beta), \\
w_{0}(\alpha, \beta, t) & \approx \sum_{r=1}^{m} \bar{w}_{0}^{r}(t) H_{r}^{e}(\alpha, \beta), & \phi(\alpha, \beta, t) & \approx \bar{\phi}(t) .
\end{aligned}
$$

where $\left(\bar{u}_{0}^{j}, \bar{v}_{0}^{j}, \bar{\theta}_{\alpha}^{j}, \bar{\theta}_{\beta}^{j}\right)$ and $\left(\overline{\bar{\tau}}_{z \alpha}^{j}, \overline{\tilde{\tau}}_{z \alpha}^{j}, \overline{\bar{\tau}}_{\beta z}^{j}, \bar{\tau}_{\beta z}^{j}\right)$ denote the values of the generalized in-plane displacements, rotations and surface shear stresses at the $j$ th node of the Lagrange elements, $\bar{w}_{0}^{r}$ denote the values of $w_{0}$ and its derivatives with respect to $\alpha$ and $\beta$ at the nodes of the Hermite elements, and $L_{j}^{e}$ and $H_{r}^{e}$ are the Lagrange and Hermite elemental interpolation functions, respectively. For the conforming fournoded rectangular element $(n=4$ and $m=12)$ the total number of DoFs per element is 49 and for the nonconforming is 45 .

\section{Discrete Finite Element Equations of the Shell Layer}

Substituting the spatial approximations of the generalized displacements, surface stresses and electric potential difference in Eqs. (80) into the weak forms in Eqs. (61)-(70), the $i$ th equation associated with each weak form is given as

$$
\begin{aligned}
& \sum_{j=1}^{n}\left(M_{i j}^{11} \ddot{\bar{u}}_{0}^{j}+M_{i j}^{14} \ddot{\bar{\theta}}_{\alpha}^{j}+M_{i j}^{16} \ddot{\bar{\tau}}_{z \alpha}^{j}+M_{i j}^{17} \ddot{\tilde{\tau}}_{z \alpha}^{j}+K_{i j}^{11} \bar{u}_{0}^{j}+K_{i j}^{12} \bar{v}_{0}^{j}+K_{i j}^{14} \bar{\theta}_{\alpha}^{j}+K_{i j}^{15} \bar{\theta}_{\beta}^{j}\right. \\
&+\left.K_{i j}^{16} \bar{\tau}_{z \alpha}^{j}+K_{i j}^{17} \overline{\tilde{\tau}}_{z \alpha}^{j}+K_{i j}^{18} \bar{\tau}_{\beta z}^{j}+K_{i j}^{19} \overline{\tilde{\tau}}_{\beta z}^{j}\right)+\sum_{r=1}^{m}\left(M_{i r}^{13} \ddot{\bar{w}}_{0}^{r}+K_{i r}^{13} \bar{w}_{0}^{r}\right)+K_{i \phi}^{1} \bar{\phi}-F_{i}^{1}=0 \\
& \sum_{j=1}^{n}\left(M_{i j}^{22} \ddot{\bar{v}}_{0}^{j}+M_{i j}^{25} \ddot{\bar{\theta}}_{\beta}^{j}+M_{i j}^{28} \ddot{\bar{\tau}}_{\beta z}^{j}+M_{i j}^{29} \ddot{\tilde{\tilde{\tau}}}_{\beta z}^{j}+K_{i j}^{21} \bar{u}_{0}^{j}+K_{i j}^{22} \bar{v}_{0}^{j}+K_{i j}^{24} \bar{\theta}_{\alpha}^{j}+K_{i j}^{25} \bar{\theta}_{\beta}^{j}\right. \\
&+\left.K_{i j}^{26} \overline{\bar{\tau}}_{z \alpha}^{j}+K_{i j}^{27} \overline{\tilde{\tau}}_{z \alpha}^{j}+K_{i j}^{28} \bar{\tau}_{\beta z}^{j}+K_{i j}^{29} \overline{\tilde{\tau}}_{\beta z}^{j}\right)+\sum_{r=1}^{m}\left(M_{i r}^{23} \ddot{\ddot{w}}_{0}^{r}+K_{i r}^{23} \bar{w}_{0}^{r}\right)+K_{i \phi}^{2} \bar{\phi}-F_{i}^{2}=0 \\
& \sum_{j=1}^{n}\left(M_{r j}^{31} \ddot{\bar{u}}_{0}^{j}+M_{r j}^{32} \ddot{\bar{v}}_{0}^{j}+M_{r j}^{34} \ddot{\bar{\theta}}_{\alpha}^{j}+M_{r j}^{35} \ddot{\bar{\theta}}_{\beta}^{j}+M_{r j}^{36} \overline{\bar{\tau}}_{z \alpha}^{j}+M_{r j}^{37} \ddot{\tilde{\tau}}_{z \alpha}^{j}+M_{r j}^{38} \ddot{\bar{\tau}}_{\beta z}^{j}+M_{r j}^{39} \ddot{\tilde{\tau}}_{\beta z}^{j}+K_{r j}^{31} \bar{u}_{0}^{j}+K_{r j}^{32} \bar{v}_{0}^{j}\right. \\
&\left.+K_{r j}^{34} \bar{\theta}_{\alpha}^{j}+K_{r j}^{35} \bar{\theta}_{\beta}^{j}+K_{r j}^{36} \overline{\bar{\tau}}_{z \alpha}^{j}+K_{r j}^{37} \overline{\tilde{\tau}}_{z \alpha}^{j}+K_{r j}^{38} \overline{\bar{\tau}}_{\beta z}^{j}+K_{r j}^{39} \bar{\tau}_{\beta z}^{j}\right)+\sum_{s=1}^{m}\left(M_{r s}^{33} \ddot{\bar{w}}_{0}^{s}+K_{r s}^{33} \bar{w}_{0}^{s}\right)+K_{r \phi}^{3} \bar{\phi}-F_{r}^{3}=0
\end{aligned}
$$




$$
\begin{aligned}
& \sum_{j=1}^{n}\left(M_{i j}^{41} \ddot{\bar{u}}_{0}^{j}+M_{i j}^{44} \ddot{\bar{\theta}}_{\alpha}^{j}+M_{i j}^{46} \ddot{\bar{\tau}}_{z \alpha}^{j}+M_{i j}^{47} \ddot{\tilde{\tau}}_{z \alpha}^{j}+K_{i j}^{41} \bar{u}_{0}^{j}+K_{i j}^{42} \bar{v}_{0}^{j}+K_{i j}^{44} \bar{\theta}_{\alpha}^{j}+K_{i j}^{45} \bar{\theta}_{\beta}^{j}\right. \\
& \left.+K_{i j}^{46} \bar{\tau}_{z \alpha}^{j}+K_{i j}^{47} \overline{\tilde{\tau}}_{z \alpha}^{j}+K_{i j}^{48} \overline{\bar{\tau}}_{\beta z}^{j}+K_{i j}^{49} \overline{\tilde{\tau}}_{\beta z}^{j}\right)+\sum_{r=1}^{m}\left(M_{i r}^{43} \ddot{\bar{w}}_{0}^{r}+K_{i r}^{43} \bar{w}_{0}^{r}\right)+K_{i \phi}^{4} \bar{\phi}-F_{i}^{4}=0, \\
& \sum_{j=1}^{n}\left(M_{i j}^{52} \ddot{\bar{v}}_{0}^{j}+M_{i j}^{55} \ddot{\bar{\theta}}_{\beta}^{j}+M_{i j}^{58} \ddot{\bar{\tau}}_{\beta z}^{j}+M_{i j}^{59} \ddot{\tilde{\tau}}_{\beta z}^{j}+K_{i j}^{51} \bar{u}_{0}^{j}+K_{i j}^{52} \bar{v}_{0}^{j}+K_{i j}^{54} \bar{\theta}_{\alpha}^{j}+K_{i j}^{55} \bar{\theta}_{\beta}^{j}\right. \\
& \left.+K_{i j}^{56} \overline{\bar{\tau}}_{z \alpha}^{j}+K_{i j}^{57} \overline{\tilde{\tau}}_{z \alpha}^{j}+K_{i j}^{58} \overline{\bar{\tau}}_{\beta z}^{j}+K_{i j}^{59} \overline{\tilde{\tau}}_{\beta z}^{j}\right)+\sum_{r=1}^{m}\left(M_{i r}^{53} \ddot{\bar{w}}_{0}^{r}+K_{i r}^{53} \bar{w}_{0}^{r}\right)+K_{i \phi}^{5} \bar{\phi}-F_{i}^{5}=0, \\
& \sum_{j=1}^{n}\left(M_{i j}^{61} \ddot{\bar{u}}_{0}^{j}+M_{i j}^{64} \ddot{\bar{\theta}}_{\alpha}^{j}+M_{i j}^{66} \ddot{\bar{\tau}}_{z \alpha}^{j}+M_{i j}^{67} \ddot{\tilde{\tau}}_{z \alpha}^{j}+K_{i j}^{61} \bar{u}_{0}^{j}+K_{i j}^{62} \bar{v}_{0}^{j}+K_{i j}^{64} \bar{\theta}_{\alpha}^{j}+K_{i j}^{65} \bar{\theta}_{\beta}^{j}\right. \\
& \left.+K_{i j}^{66} \overline{\bar{\tau}}_{z \alpha}^{j}+K_{i j}^{67} \overline{\tilde{\tau}}_{z \alpha}^{j}+K_{i j}^{68} \overline{\bar{\tau}}_{\beta z}^{j}+K_{i j}^{69} \overline{\tilde{\tau}}_{\beta z}^{j}\right)+\sum_{r=1}^{m}\left(M_{i r}^{63} \ddot{\bar{w}}_{0}^{r}+K_{i r}^{63} \bar{w}_{0}^{r}\right)+K_{i \phi}^{6} \bar{\phi}-F_{i}^{6}=0, \\
& \sum_{j=1}^{n}\left(M_{i j}^{71} \ddot{\bar{u}}_{0}^{j}+M_{i j}^{74} \ddot{\bar{\theta}}_{\alpha}^{j}+M_{i j}^{76} \ddot{\bar{\tau}}_{z \alpha}^{j}+M_{i j}^{77} \ddot{\tilde{\tau}}_{z \alpha}^{j}+K_{i j}^{71} \bar{u}_{0}^{j}+K_{i j}^{72} \bar{v}_{0}^{j}+K_{i j}^{74} \bar{\theta}_{\alpha}^{j}+K_{i j}^{75} \bar{\theta}_{\beta}^{j}\right. \\
& \left.+K_{i j}^{76} \overline{\bar{\tau}}_{z \alpha}^{j}+K_{i j}^{77} \overline{\tilde{\tau}}_{z \alpha}^{j}+K_{i j}^{78} \overline{\bar{\tau}}_{\beta z}^{j}+K_{i j}^{79} \overline{\tilde{\tau}}_{\beta z}^{j}\right)+\sum_{r=1}^{m}\left(M_{i r}^{73} \ddot{\bar{w}}_{0}^{r}+K_{i r}^{73} \bar{w}_{0}^{r}\right)+K_{i \phi}^{7} \bar{\phi}-F_{i}^{7}=0, \\
& \sum_{j=1}^{n}\left(M_{i j}^{82} \ddot{\ddot{v}}_{0}^{j}+M_{i j}^{85} \ddot{\bar{\theta}}_{\beta}^{j}+M_{i j}^{88} \ddot{\bar{\tau}}_{\beta z}^{j}+M_{i j}^{89} \ddot{\tilde{\tau}}_{\beta z}^{j}+K_{i j}^{81} \bar{u}_{0}^{j}+K_{i j}^{82} \bar{v}_{0}^{j}+K_{i j}^{84} \bar{\theta}_{\alpha}^{j}+K_{i j}^{85} \bar{\theta}_{\beta}^{j}\right. \\
& \left.+K_{i j}^{86} \overline{\bar{\tau}}_{z \alpha}^{j}+K_{i j}^{87} \overline{\tilde{\tau}}_{z \alpha}^{j}+K_{i j}^{88} \overline{\bar{\tau}}_{\beta z}^{j}+K_{i j}^{89} \overline{\tilde{\tau}}_{\beta z}^{j}\right)+\sum_{r=1}^{m}\left(M_{i r}^{83} \ddot{\bar{w}}_{0}^{r}+K_{i r}^{83} \bar{w}_{0}^{r}\right)+K_{i \phi}^{8} \bar{\phi}-F_{i}^{8}=0, \\
& \sum_{j=1}^{n}\left(M_{i j}^{92} \ddot{\bar{v}}_{0}^{j}+M_{i j}^{95} \ddot{\bar{\theta}}_{\beta}^{j}+M_{i j}^{98} \ddot{\bar{\tau}}_{\beta z}^{j}+M_{i j}^{99} \ddot{\tilde{\tau}}_{\beta z}^{j}+K_{i j}^{91} \bar{u}_{0}^{j}+K_{i j}^{92} \bar{v}_{0}^{j}+K_{i j}^{94} \bar{\theta}_{\alpha}^{j}+K_{i j}^{95} \bar{\theta}_{\beta}^{j}\right. \\
& \left.+K_{i j}^{96} \overline{\bar{\tau}}_{z \alpha}^{j}+K_{i j}^{97} \overline{\tilde{\tau}}_{z \alpha}^{j}+K_{i j}^{98} \overline{\bar{\tau}}_{\beta z}^{j}+K_{i j}^{99} \overline{\tilde{\tau}}_{\beta z}^{j}\right)+\sum_{r=1}^{m}\left(M_{i r}^{93} \ddot{\bar{w}}_{0}^{r}+K_{i r}^{93} \bar{w}_{0}^{r}\right)+K_{i \phi}^{9} \bar{\phi}-F_{i}^{9}=0,
\end{aligned}
$$

where $i=1, \ldots, n$, and $r=1, \ldots, m$. Regarding the weak form of the equation governing the electrostatic equilibrium in Eq. (70) yields

$$
K_{\phi i}^{1} \bar{u}_{0}^{i}+K_{\phi i}^{2} \bar{v}_{0}^{i}+K_{\phi r}^{3} \bar{w}_{0}^{r}+K_{\phi i}^{4} \bar{\theta}_{\alpha}^{i}+K_{\phi i}^{5} \bar{\theta}_{\beta}^{i}+K_{\phi i}^{6} \overline{\bar{\tau}}_{z \alpha}^{i}+K_{\phi i}^{7} \overline{\tilde{\tau}}_{z \alpha}^{i}+K_{\phi i}^{8} \overline{\bar{\tau}}_{\beta z}^{i}+K_{\phi i}^{9} \overline{\tilde{\tau}}_{\beta z}^{j}+K_{\phi \phi} \bar{\phi}-Q_{\phi}=0 .
$$

The coefficients of the mass matrix $M_{i j}^{x y}=M_{j i}^{y x}$, stiffness matrix $K_{i j}^{x y}=K_{j i}^{y x}$, piezoelectric coupling matrix $K_{i \phi}^{x}=K_{\phi i}^{x}$, capacitance matrix $K_{\phi \phi}$, force vectors $F_{i}^{x}$ and electric charge $Q_{\phi}$ are defined in Appendix H.

In matrix notation Eqs. (81)-(90) can be expressed in terms of the elemental matrices and vectors of the generic layer $l$ as

$$
\begin{aligned}
& {\left[\begin{array}{ll}
\mathbf{M}_{u u}^{l} & \mathbf{M}_{u \tau}^{l} \\
\mathbf{M}_{\tau u}^{l} & \mathbf{M}_{\tau \tau}^{l}
\end{array}\right]\left\{\begin{array}{c}
\ddot{\overline{\mathbf{u}}}^{l}(t) \\
\ddot{\bar{\tau}}^{l}(t)
\end{array}\right\}+\left[\begin{array}{ll}
\mathbf{K}_{u u}^{l} & \mathbf{K}_{u \tau}^{l} \\
\mathbf{K}_{\tau u}^{l} & \mathbf{K}_{\tau \tau}^{l}
\end{array}\right]\left\{\begin{array}{c}
\overline{\mathbf{u}}^{l}(t) \\
\overline{\boldsymbol{\tau}}^{l}(t)
\end{array}\right\}+\left\{\begin{array}{c}
\mathbf{K}_{u \phi}^{l} \\
\mathbf{K}_{\tau \phi}^{l}
\end{array}\right\} \bar{\phi}(t)=\left\{\begin{array}{c}
\mathbf{F}_{u}^{l}(t) \\
\mathbf{F}_{\tau}^{l}(t)
\end{array}\right\},} \\
& \left\{\begin{array}{ll}
\mathbf{K}_{\phi u}^{l} & \mathbf{K}_{\tau \phi}^{l}
\end{array}\right\}\left\{\begin{array}{c}
\overline{\mathbf{u}}^{l}(t) \\
\overline{\boldsymbol{\tau}}^{l}(t)
\end{array}\right\}+K_{\phi \phi} \bar{\phi}(t)=Q_{\phi}(t)
\end{aligned}
$$


where, since $\mathbf{K}^{y x}=\left(\mathbf{K}^{x y}\right)^{\mathrm{T}}$ and $\mathbf{M}^{y x}=\left(\mathbf{M}^{x y}\right)^{\mathrm{T}}$, one gets $\mathbf{M}_{\tau u}^{l}=\left(\mathbf{M}_{u \tau}^{l}\right)^{\mathrm{T}}, \mathbf{K}_{\tau u}^{l}=\left(\mathbf{K}_{u \tau}^{l}\right)^{\mathrm{T}}, \mathbf{K}_{\phi u}^{l}=\left(\mathbf{K}_{u \phi}^{l}\right)^{\mathrm{T}}$ and $\mathbf{K}_{\tau \phi}^{l}=\left(\mathbf{K}_{\tau \phi}^{l}\right)^{\mathrm{T}}$, and the matrices and vectors are

$$
\begin{aligned}
& \mathbf{M}_{u u}^{l}=\left[\begin{array}{ccccc}
\mathbf{M}^{11} & \mathbf{0} & \mathbf{M}^{13} & \mathbf{M}^{14} & \mathbf{0} \\
\mathbf{0} & \mathbf{M}^{22} & \mathbf{M}^{23} & \mathbf{0} & \mathbf{M}^{25} \\
\mathbf{M}^{31} & \mathbf{M}^{32} & \mathbf{M}^{33} & \mathbf{M}^{34} & \mathbf{M}^{35} \\
\mathbf{M}^{41} & \mathbf{0} & \mathbf{M}^{43} & \mathbf{M}^{44} & \mathbf{0} \\
\mathbf{0} & \mathbf{M}^{52} & \mathbf{M}^{53} & \mathbf{0} & \mathbf{M}^{55}
\end{array}\right], \quad \mathbf{K}_{u u}^{l}=\left[\begin{array}{lllll}
\mathbf{K}^{11} & \mathbf{K}^{12} & \mathbf{K}^{13} & \mathbf{K}^{14} & \mathbf{K}^{15} \\
\mathbf{K}^{21} & \mathbf{K}^{22} & \mathbf{K}^{23} & \mathbf{K}^{24} & \mathbf{K}^{25} \\
\mathbf{K}^{31} & \mathbf{K}^{32} & \mathbf{K}^{33} & \mathbf{K}^{34} & \mathbf{K}^{35} \\
\mathbf{K}^{41} & \mathbf{K}^{42} & \mathbf{K}^{43} & \mathbf{K}^{44} & \mathbf{K}^{45} \\
\mathbf{K}^{51} & \mathbf{K}^{52} & \mathbf{K}^{53} & \mathbf{K}^{54} & \mathbf{K}^{55}
\end{array}\right] \\
& \mathbf{M}_{u \tau}^{l}=\left[\begin{array}{cccc}
\mathbf{M}^{16} & \mathbf{M}^{17} & \mathbf{0} & \mathbf{0} \\
\mathbf{0} & \mathbf{0} & \mathbf{M}^{28} & \mathbf{M}^{29} \\
\mathbf{M}^{36} & \mathbf{M}^{37} & \mathbf{M}^{38} & \mathbf{M}^{39} \\
\mathbf{M}^{46} & \mathbf{M}^{47} & \mathbf{0} & \mathbf{0} \\
\mathbf{0} & \mathbf{0} & \mathbf{M}^{58} & \mathbf{M}^{59}
\end{array}\right], \quad \mathbf{K}_{u \tau}^{l}=\left[\begin{array}{cccc}
\mathbf{M}^{16} & \mathbf{M}^{17} & \mathbf{0} & \mathbf{0} \\
\mathbf{0} & \mathbf{0} & \mathbf{M}^{28} & \mathbf{M}^{29} \\
\mathbf{M}^{36} & \mathbf{M}^{37} & \mathbf{M}^{38} & \mathbf{M}^{39} \\
\mathbf{M}^{46} & \mathbf{M}^{47} & \mathbf{0} & \mathbf{0} \\
\mathbf{0} & \mathbf{0} & \mathbf{M}^{58} & \mathbf{M}^{59}
\end{array}\right], \quad \overline{\mathbf{u}}^{l}(t)=\left\{\begin{array}{l}
\overline{\mathbf{u}}_{0} \\
\overline{\mathbf{v}}_{0} \\
\overline{\mathbf{w}}_{0} \\
\overline{\boldsymbol{\theta}}_{\alpha} \\
\overline{\boldsymbol{\theta}}_{\beta}
\end{array}\right\} \\
& \mathbf{M}_{\tau \tau}^{l}=\left[\begin{array}{cccc}
\mathbf{M}^{66} & \mathbf{M}^{67} & \mathbf{0} & \mathbf{0} \\
\mathbf{M}^{76} & \mathbf{M}^{77} & \mathbf{0} & \mathbf{0} \\
\mathbf{0} & \mathbf{0} & \mathbf{M}^{88} & \mathbf{M}^{89} \\
\mathbf{0} & \mathbf{0} & \mathbf{M}^{98} & \mathbf{M}^{99}
\end{array}\right], \quad \mathbf{M}_{\tau \tau}^{l}=\left[\begin{array}{cccc}
\mathbf{K}^{66} & \mathbf{K}^{67} & \mathbf{K}^{68} & \mathbf{K}^{69} \\
\mathbf{K}^{76} & \mathbf{K}^{77} & \mathbf{K}^{78} & \mathbf{K}^{79} \\
\mathbf{K}^{86} & \mathbf{K}^{87} & \mathbf{K}^{88} & \mathbf{K}^{89} \\
\mathbf{K}^{96} & \mathbf{K}^{97} & \mathbf{K}^{98} & \mathbf{K}^{99}
\end{array}\right], \quad \overline{\boldsymbol{\tau}}^{l}(t)=\left\{\begin{array}{l}
\overline{\overline{\boldsymbol{\tau}}}_{z \alpha} \\
\overline{\tilde{\boldsymbol{\tau}}}_{z \alpha} \\
\overline{\overline{\boldsymbol{\tau}}}_{\beta z} \\
\tilde{\tilde{\boldsymbol{\tau}}}_{\beta z}
\end{array}\right\}, \\
& \mathbf{K}_{u \phi}^{l}=\left\{\begin{array}{c}
\mathbf{K}_{\phi}^{1} \\
\mathbf{K}_{\phi}^{2} \\
\mathbf{K}_{\phi}^{3} \\
\mathbf{K}_{\phi}^{4} \\
\mathbf{K}_{\phi}^{5}
\end{array}\right\}, \quad \mathbf{F}_{u}^{l}(t)=\left\{\begin{array}{c}
\mathbf{F}^{1} \\
\mathbf{F}^{2} \\
\mathbf{F}^{3} \\
\mathbf{F}^{4} \\
\mathbf{F}^{5}
\end{array}\right\}, \quad \mathbf{K}_{\tau \phi}^{l}=\left\{\begin{array}{l}
\mathbf{K}_{\phi}^{6} \\
\mathbf{K}_{\phi}^{7} \\
\mathbf{K}_{\phi}^{8} \\
\mathbf{K}_{\phi}^{9}
\end{array}\right\}, \quad \mathbf{F}_{\tau}^{l}(t)=\left\{\begin{array}{l}
\mathbf{F}^{6} \\
\mathbf{F}^{7} \\
\mathbf{F}^{8} \\
\mathbf{F}^{9}
\end{array}\right\} .
\end{aligned}
$$

The coupled "mixed" electro-mechanical FE model in Eqs. (91) and (92) is based on the weak forms of the equations of motion and electrostatic equilibrium, which where obtained through Hamilton's principle, and is expressed in terms of displacement (and the derivatives of the transverse displacement), surface shear stresses and electric potential difference DoFs, which are all coupled. It should be noted that the contributions of the internal forces defined in vectors $\mathbf{F}_{u}^{l}$ and $\mathbf{F}_{\tau}^{l}$ to the force vector will cancel when element equations are assembled. They will remain in the force vector only when the element boundary coincides with the boundary of the domain being modeled. However, as is well known, the contributions of the distributed applied loads $Z(\alpha, \beta)$ to a node will add up from elements connected at the node and remain as a part of the force vector (see Reddy [107, pp. 313-318]). Regarding the electric potential difference it is assumed constant in each element and, as can be seen in Eq. (91), it produces an equivalent mechanical load though the $\mathbf{K}_{u \phi}^{l}$ and $\mathbf{K}_{\tau \phi}^{l}$ vectors. Regarding the Eq. (92), associated with the sensing capabilities os the piezoelectric shell layer, it establishes the electrostatic relation at the shell layer FE level between the strains (related with the "mixed" displacements), induced electric potential difference and surface electric charge.

\section{Assemblage of Matrices from Layer to Multilayer Level}

In this section the elemental equations derived for the generic single shell layer are adapted in order to allow the generalization of the present theory to a multilayer, or discrete layer, type formulation. To that end, since the displacements DoFs of the elemental equations of the shell layer are defined in terms of in-plane generalized displacements in the middle surface and rotations of the normals to the middle surface, first the DoFs are transformed to equivalent in-plane displacements on the top and bottom surfaces of the generic shell layer element. Additionally, since the effects of the surface top and bottom shear stresses have been represented in terms of mean and relative quantities, another transformation is required to the stress DoFs to dispose of top and bottom shear stresses DoFs. The transverse displacement is assumed constant in the multilayer shell (i.e., is constant, and the same, for all layers). These transformations allow the displacement and stress DoFs of different layers to be assembled imposing not only displacement continuity but also shear stress continuity across the interfaces of the multilayer shell FE. Thus, the FE is "regenerated" (in opposition 
to the well-known "degeneration" approach) in the form of an equivalent eight-noded 3-D element with 2 inplane displacement and 2 shear stress DoFs per node, and one transverse displacement (and its derivatives) and one electric potential difference per element. Therefore, the "regenerated" formulation is suitable for assemblage of elemental matrices from single layer to multilayer level.

The effects of the pairs of generalized variables $\left(u_{0}, \theta_{\alpha}\right)$ and $\left(v_{0}, \theta_{\beta}\right)$ in the global displacement field are taken into account through new equivalent pairs of generalized variables $\left(u_{t}, u_{b}\right)$ and $\left(v_{t}, v_{b}\right)$, with each pair containing the in-plane translations at the top and bottom surfaces, respectively. Thus, rather than describing the in-plane displacement field by a translation and a rotation at one point, it can more conveniently be described here by the translation at two points on the top and bottom surfaces.

According to Eq. (44), and using the adequate coefficients of matrices $\mathbf{z}^{u}(z=h)$ and $\mathbf{z}^{\tau}(z=h)$, the displacement field $u(\alpha, \beta, z)$ on the top surface is given as

$$
u_{t}=z_{11}^{u}(h) u_{0}+z_{13}^{u}(h) \frac{\partial w_{0}}{\partial \alpha}+z_{14}^{u}(h) \theta_{\alpha}+z_{11}^{\tau}(h) \bar{\tau}_{z \alpha}+z_{12}^{\tau}(h) \tilde{\tau}_{z \alpha} .
$$

Then, from the previous equation $u_{0}=u_{0}(\alpha, \beta)$ is written as

$$
u_{0}=\frac{1}{z_{11}^{u}(h)} u_{t}-\frac{z_{13}^{u}(h)}{z_{11}^{u}(h)} \frac{\partial w_{0}}{\partial \alpha}-\frac{z_{14}^{u}(h)}{z_{11}^{u}(h)} \theta_{\alpha}-\frac{z_{11}^{\tau}(h)}{z_{11}^{u}(h)} \bar{\tau}_{z \alpha}-\frac{z_{12}^{\tau}(h)}{z_{11}^{u}(h)} \tilde{\tau}_{z \alpha} .
$$

Substituting the definition of $u_{0}=u_{0}(\alpha, \beta, z=0)$ in terms of $u_{t}=u_{t}(\alpha, \beta, z=h)$ yields

$$
\begin{aligned}
u & =\frac{z_{11}^{u}}{z_{11}^{u}(h)} u_{t}+\left[z_{13}^{u}-z_{11}^{u} \frac{z_{13}^{u}(h)}{z_{11}^{u}(h)}\right] \frac{\partial w_{0}}{\partial \alpha}+\left[z_{14}^{u}-z_{11}^{u} \frac{z_{14}^{u}(h)}{z_{11}^{u}(h)}\right] \theta_{\alpha} \\
& +\left[z_{11}^{\tau}-z_{11}^{u} \frac{z_{11}^{\tau}(h)}{z_{11}^{u}(h)}\right] \bar{\tau}_{z \alpha}+\left[z_{12}^{\tau}-z_{11}^{u} \frac{z_{12}^{\tau}(h)}{z_{11}^{u}(h)}\right] \tilde{\tau}_{z \alpha} .
\end{aligned}
$$

From the previous equation it can be seen that some transformations to the first line of matrices $\mathbf{z}^{u}(z)$ and $\mathbf{z}^{\tau}(z)$ was performed in order to make a transformation of the generalized in-plane displacement $u_{0}$ on the middle surface to the translation on the top surface $u_{t}$. Performing a similar process to eliminate the rotation $\theta_{\alpha}$ of Eq. (96) and express the displacement $u$ also in terms of the translation on the bottom surface $u_{b}$, another transformation is performed considering also the terms of the first line of $\mathbf{z}^{u}(z=-h)$ and $\mathbf{z}^{\tau}(z=-h)$. Similar relations hold for the second pair of variables $\left(v_{0}, \theta_{\beta}\right)$. For the sake of brevity the algebra of these relations will not be be presented here but can easily be derived from the previous explanation.

Other required transformation to "regenerate" the 2-D element is performed according to Eqs. (4) and (5), where relationships between the mean and relative shear stresses and the shear stresses on the interfaces of the generic shell layer $\sigma_{z \alpha}^{t}, \sigma_{z \alpha}^{b}, \sigma_{\beta z}^{t}$ and $\sigma_{\beta z}^{b}$ can be easily established.

According to the previous discussion, the relationship between the original and "regenerated" set of generalized variables used to defined the in-plane displacement field can be established by means of a transformation matrices $\mathbf{T}_{u}$ and $\mathbf{T}_{\tau}$ as

$$
\left\{\begin{array}{l}
\overline{\mathbf{u}}_{0} \\
\overline{\mathbf{v}}_{0} \\
\overline{\mathbf{w}}_{0} \\
\overline{\boldsymbol{\theta}}_{\alpha} \\
\overline{\boldsymbol{\theta}}_{\beta}
\end{array}\right\}=\mathbf{T}_{u}\left\{\begin{array}{l}
\overline{\mathbf{u}}_{t} \\
\overline{\mathbf{u}}_{b} \\
\overline{\mathbf{v}}_{t} \\
\overline{\mathbf{v}}_{b} \\
\overline{\mathbf{w}}
\end{array}\right\}, \quad\left\{\begin{array}{l}
\overline{\overline{\boldsymbol{\tau}}}_{z \alpha} \\
\overline{\tilde{\boldsymbol{\tau}}}_{z \alpha} \\
\overline{\overline{\boldsymbol{\tau}}}_{\beta z} \\
\overline{\tilde{\boldsymbol{\tau}}}_{\beta z}
\end{array}\right\}=\mathbf{T}_{\tau}\left\{\begin{array}{l}
\overline{\boldsymbol{\sigma}}_{z \alpha}^{t} \\
\overline{\boldsymbol{\sigma}}_{z \alpha}^{b} \\
\overline{\boldsymbol{\sigma}}_{\beta z}^{t} \\
\overline{\boldsymbol{\sigma}}_{\beta z}^{b}
\end{array}\right\} .
$$

Performing the previous transformations into the FE elemental matrices in Eqs. (91) and (92), where the elemental matrices and vectors are transformed according to (similar relations hold for the stiffness matrices)

$$
\begin{gathered}
\mathbf{M}_{u u}^{* l}=\mathbf{T}_{u}^{\mathrm{T}} \mathbf{M}_{u u}^{l} \mathbf{T}_{u}, \quad \mathbf{M}_{\tau \tau}^{* l}=\mathbf{T}_{u}^{\mathrm{T}} \mathbf{M}_{\tau \tau}^{l} \mathbf{T}_{u}, \quad \mathbf{M}_{u \tau}^{* l}=\mathbf{T}_{u}^{\mathrm{T}} \mathbf{M}_{u \tau}^{l} \mathbf{T}_{\tau}, \\
\mathbf{K}_{u \phi}^{* l}=\mathbf{T}_{u}^{\mathrm{T}} \mathbf{K}_{u \phi}^{l}, \quad \mathbf{K}_{\tau \phi}^{* l}=\mathbf{T}_{\tau} \mathbf{K}_{\tau \phi}^{l}, \quad \mathbf{F}_{u}^{* l}=\mathbf{T}_{u}^{\mathrm{T}} \mathbf{F}_{u}^{l},
\end{gathered}
$$


yields

$$
\begin{gathered}
{\left[\begin{array}{ll}
\mathbf{M}_{u u}^{* l} & \mathbf{M}_{u \tau}^{* l} \\
\mathbf{M}_{\tau u}^{* l} & \mathbf{M}_{\tau \tau}^{* l}
\end{array}\right]\left\{\begin{array}{c}
\ddot{\overline{\mathbf{u}}}^{* l}(t) \\
\ddot{\bar{\tau}}^{* l}(t)
\end{array}\right\}+\left[\begin{array}{ll}
\mathbf{K}_{u u}^{* l} & \mathbf{K}_{u \tau}^{* l} \\
\mathbf{K}_{\tau u}^{* l} & \mathbf{K}_{\tau \tau}^{* l}
\end{array}\right]\left\{\begin{array}{c}
\overline{\mathbf{u}}^{* l}(t) \\
\overline{\boldsymbol{\tau}}^{* l}(t)
\end{array}\right\}+\left\{\begin{array}{l}
\mathbf{K}_{u \phi}^{* l} \\
\mathbf{K}_{\tau \phi}^{* l}
\end{array}\right\} \bar{\phi}(t)=\left\{\begin{array}{l}
\mathbf{F}_{u}^{* l}(t) \\
\mathbf{F}_{\tau}^{* l}(t)
\end{array}\right\},} \\
\left\{\begin{array}{ll}
\mathbf{K}_{\phi u}^{* l} & \mathbf{K}_{\tau \phi}^{* l}
\end{array}\right\}\left\{\begin{array}{l}
\overline{\mathbf{u}}^{* l}(t) \\
\overline{\boldsymbol{\tau}}^{* l}(t)
\end{array}\right\}+K_{\phi \phi} \bar{\phi}(t)=Q_{\phi}(t),
\end{gathered}
$$

From this point forward, the generic layer elemental matrices can be assembled in the thickness direction in order to create the desired multilayer FE according to the representative multilayer shell model to be generated. Displacement and shear stress continuity at the through-the-thickness interfaces of adjacent elements (discrete layers) is imposed in the assemblage process, as is usually done with the displacement DoF of 3-D elements, and it is assumed that no slippage occurs in the interfaces between adjacent layers. It is worthy to mention that the resultant multilayer elemental matrices are needed, for example, when there is the need to consider segmented layers, as is the case when dealing with arbitrary damping treatments (piezoelectric or viscoelastic patches) mounted on a host shell structure. After the through-the-thickness assemblage, the multilayer elemental matrices are written as

$$
\begin{gathered}
{\left[\begin{array}{ll}
\mathbf{M}_{u u}^{e} & \mathbf{M}_{u \tau}^{e} \\
\mathbf{M}_{\tau u}^{e} & \mathbf{M}_{\tau \tau}^{e}
\end{array}\right]\left\{\begin{array}{c}
\ddot{\mathbf{u}}^{e}(t) \\
\ddot{\bar{\tau}}^{e}(t)
\end{array}\right\}+\left[\begin{array}{ll}
\mathbf{K}_{u u}^{e} & \mathbf{K}_{u \tau}^{e} \\
\mathbf{K}_{\tau u}^{e} & \mathbf{K}_{\tau \tau}^{e}
\end{array}\right]\left\{\begin{array}{c}
\overline{\mathbf{u}}^{e}(t) \\
\overline{\boldsymbol{\tau}}^{e}(t)
\end{array}\right\}+\left\{\begin{array}{l}
\mathbf{K}_{u \phi}^{e} \\
\mathbf{K}_{\tau \phi}^{e}
\end{array}\right\} \bar{\phi}^{e}(t)=\left\{\begin{array}{l}
\mathbf{F}_{u}^{e}(t) \\
\mathbf{F}_{\tau}^{e}(t)
\end{array}\right\},} \\
\left\{\begin{array}{ll}
\mathbf{K}_{\phi u}^{e} & \mathbf{K}_{\tau \phi}^{e}
\end{array}\right\}\left\{\begin{array}{l}
\overline{\mathbf{u}}^{e}(t) \\
\overline{\boldsymbol{\tau}}^{e}(t)
\end{array}\right\}+\mathbf{K}_{\phi \phi}^{e} \bar{\phi}^{e}(t)=\mathbf{Q}_{\phi}^{e}(t),
\end{gathered}
$$

where the superscript $(\cdot)^{e}$ is used to denote multilayer elemental matrices and vectors. Regarding the electrical part of the FE equations, It should be noted that since the multilayer shell can contain several piezoelectric layers, there is more than one electrical DoF and therefore instead of a single electrical potential we have a vector $\bar{\phi}^{e}$ of electrical potential differences and the correspondent capacitance matrices $\mathbf{K}_{\phi \phi}^{e}$ and electric charge vector $\mathbf{Q}_{\phi}^{e}$.

Assuming homogeneous shear stress conditions on the free top and bottom surfaces of the multilayer shell element and performing a dynamic condensation to the shear stress DoFs, as suggested for a generic system, for example, by Kidder ${ }^{108}$ or $\mathrm{O}^{\prime}$ Callahan ${ }^{109}$ and Gordis, ${ }^{110}$ yields the multilayer FE elemental equations in terms of elemental reduced matrices and vectors in terms of only displacement variables as

$$
\begin{gathered}
\hat{\mathbf{M}}_{u u}^{e} \ddot{\mathbf{u}}^{e}(t)+\hat{\mathbf{K}}_{u u}^{e} \overline{\mathbf{u}}^{e}(t)+\hat{\mathbf{K}}_{u \phi}^{e} \overline{\boldsymbol{\phi}}^{e}(t)=\hat{\mathbf{F}}_{u}^{e}(t), \\
\hat{\mathbf{K}}_{\phi u}^{e} \overline{\mathbf{u}}^{e}(t)+\mathbf{K}_{\phi \phi}^{e} \overline{\boldsymbol{\phi}}^{e}(t)=\mathbf{Q}_{\phi}^{e}(t) .
\end{gathered}
$$

A generic fully discretized global electro-mechanical system is obtained by "in-plane" assembling the elemental multilayer FE matrices and vectors yielding

$$
\begin{gathered}
\mathbf{M}_{u u} \ddot{\ddot{\mathbf{u}}}(t)+\mathbf{K}_{u u} \overline{\mathbf{u}}(t)+\mathbf{K}_{\phi u}^{\mathrm{T}} \overline{\boldsymbol{\phi}}(t)=\mathbf{F}_{u}(t) \\
\mathbf{K}_{\phi u} \overline{\mathbf{u}}(t)+\mathbf{K}_{\phi \phi} \overline{\boldsymbol{\phi}}(t)=\mathbf{Q}_{\phi}(t),
\end{gathered}
$$

where the superscript $(\cdot)^{e}$ and the hat above the elemental matrices and vectors have been dropped to denote global matrices and vectors of the fully discretized FE model.

The electrical DoFs vector in Eqs. (105) and (106) can be partitioned into the actuating and sensing DoFs,

$$
\bar{\phi}(t)=\operatorname{col}\left[\bar{\phi}_{\mathrm{a}}(t), \bar{\phi}_{\mathrm{s}}(t)\right]
$$

where the subscripts $(\cdot)_{\mathrm{a}}$ and $(\cdot)_{\mathrm{s}}$ denote the actuating and sensing capabilities. Furthermore, the stiffness matrix can be written as the sum of the elastic and piezoelectric layers stiffness matrices $\mathbf{K}_{u u}^{E}$ and $\mathbf{K}_{u u}^{P}$, respectively. Hence, considering open-circuit electrodes, and in that case $\mathbf{Q}_{\phi}(t)=0$ (see Vasques and Rodrigues $^{42}$ ), the non specified potential differences in (106) can be statically condensed in (105) and the equations of motion and charge equilibrium become

$$
\begin{gathered}
\mathbf{M}_{u u} \ddot{\mathbf{u}}(t)+\left(\mathbf{K}_{u u}^{E}+\mathbf{K}_{u u}^{P *}\right) \overline{\mathbf{u}}(t)=-\mathbf{K}_{\phi u a}^{\mathrm{T}} \overline{\boldsymbol{\phi}}_{\mathrm{a}}(t)+\mathbf{F}_{u}(t), \\
\overline{\boldsymbol{\phi}}_{\mathbf{s}}(t)=-\mathbf{K}_{\phi \phi \mathbf{s}}^{-1} \mathbf{K}_{\phi u s} \overline{\mathbf{u}}(t),
\end{gathered}
$$


where

$$
\mathbf{K}_{u u}^{P *}=\mathbf{K}_{u u}^{P}-\mathbf{K}_{\phi u s}^{\mathrm{T}} \mathbf{K}_{\phi \phi \mathbf{s}}^{-1} \mathbf{K}_{\phi u s} .
$$

It is worthy to mention that a through-the-thickness distribution of the induced electric potential within the piezoelectric layers was already considered in the formulation through the modification of the strain definition, as described in Sec. C, and the use of effective shear stiffness parameters in Eqs. (C12) of Appendix. Thus, the static condensation in Eq. (108) only considers the linear counterpart of the electrical potential distribution, which is the one that in fact contributes to the sensor voltage. Moreover, a second alternative where the equipotential area condition is satisfied by means of a modified static condensation of the non-specified potentials might be utilized, which corresponds to a more realistic approach, that becomes more significant for bare piezoelectric structures or as the length of the piezoelectric layers approaches the length of the host structure (see Refs. 42 and 111 for further details).

\section{E. Inclusion of Viscoelastic Damping Effects}

In this section the coupled piezo-elastic multilayer FE model in Eqs. (108) and (109) is extended to the case where the multilayer shell includes also damping layers of isotropic viscoelastic materials. In order to fully account for the viscoelastic damping effects in the FE model, the temperature and frequency dependent material properties of the viscoelastic materials cause some difficulties, increasing the complexity of the mathematical model. Usually, for simplicity, the temperature is assumed constant, and only the frequency dependent constitutive behavior is considered in the underlying models. If general transient responses are required, time domain models are suitable and versatile alternatives to frequency domain methods such as the CMA, since they allow the reduction of the computational burden due to the re-calculation of the stiffness matrix for each discrete frequency value (see, for example, Refs. 112 and 113). A good alternative is a time domain model based on a variation (or Laplace transformed) of the ADF (Anelastic Displacement Fields) model, originally developed by Lesieutre and his co-workers, ${ }^{54,55}$ as proposed by Vasques et al. ${ }^{68}$ For the sake of brevity only key steps of the derivation of the Laplace transformed variation of the ADF model will be presented here. The reader is referred to Refs. 68 and 96 for further details.

The Laplace transformed formulation of the ADF model takes a definition of the complex (frequency dependent) modulus of elasticity of the viscoelastic material in the frequency (Laplace) domain and utilizes so-called internal, or dissipation, or anelastic (after Lesieutre) variables, to simplify the equations. The use of additional variables has however the drawback of increasing the size of the problem. Afterward, through an inverse Laplace transform one obtains an amenable and computationally tractable augmented viscoelastically damped system of linear ordinary differential equations that can be solved by standard (linear) numerical procedures. With this procedure the FE model implementation of the ADF model is more straightforward when compared with the Lesieutre's original direct time domain formulation based on the methods of irreversible thermodynamics and a decomposition of the total displacement field in an elastic and anelastic counterpart.

The process of deriving an augmented coupled elastic-anelastic (using the original designation of Lesieutre) utilizes the definition of the material modulus function $s \tilde{G}(s)$ given by Lesieutre and his co-worker ${ }^{54,114}$ as a series of functions in the Laplace domain,

$$
s \tilde{G}(s)=G_{0}\left(1+\sum_{i=1}^{n} \frac{\Delta_{i} s}{s+\Omega_{i}}\right)
$$

where $G_{0}=\lim _{t \rightarrow \infty} G(t)$ is the relaxed (or static, or low-frequency) shear modulus, $\Omega_{i}$ is the inverse of the characteristic relaxation time at constant strain and $\Delta_{i}$ the correspondent relaxation resistance. To take into consideration the relaxation behavior, the entire ADF model itself may be comprised of several individual fields where $n$ series of ADF are used to describe the material behavior. Given measured values of the shear modulus in the form of frequency dependent complex modulus $G(j \omega)$, the relaxed shear modulus $G_{0}$ and the series of material parameters $\Delta_{i}$ and $\Omega_{i}$ can be determined using curve fitting or optimization techniques. The number of series of ADF parameters determines the accuracy of the matching of the measured data over the frequency range of interest (see Ref. 68).

Considering a piezo-visco-elastic multilayered shell, the stiffness matrices of the piezoelectric and elastic layers of the FE equation of motion in Eq. (108) are collected into a single matrix $\mathbf{K}_{u u}^{E P *}$, and the viscoelastic layers are collected into a complex stiffness matrix $\mathbf{K}_{u u}^{V}(j w)$. However, the complex shear modulus (note that assuming a frequency independent Poisson's coefficient both shear and extensional stiffness terms are 
considered in the viscoelastic stiffness matrix through the extensional and shear modulus relationship) of the viscoelastic stiffness matrix is factored out of $\mathbf{K}_{u u}^{V}(j w)$ yielding $\mathbf{K}_{u u}^{V}(j w)=G(j w) \overline{\mathbf{K}}_{u u}^{V}$. Assuming that all the viscoelastic layers have the same material, Eq. (108) follows a hereditary stress-strain law ${ }^{115}$ as

$$
\mathbf{M}_{u u} \ddot{\mathbf{u}}(\mathbf{t})+\mathbf{D}_{u u} \dot{\overline{\mathbf{u}}}(t)+\mathbf{K}_{u u}^{E P *} \overline{\mathbf{u}}(t)+G(t) \overline{\mathbf{K}}_{u u}^{V} \mathbf{u}(0)+\int_{0}^{t} G(t-\tau) \overline{\mathbf{K}}_{u u}^{V} \frac{\partial \overline{\mathbf{u}}(\tau)}{\partial \tau} d \tau=\mathbf{F}(t),
$$

where $\mathbf{F}(t)=\mathbf{F}_{u}(t)-\mathbf{K}_{\phi u a}^{\mathrm{T}} \overline{\boldsymbol{\phi}}_{\mathrm{a}}(t)$ and $\mathbf{D}_{u u}$ is a viscous proportional damping matrix. Transforming Eq. (112) to the Laplace domain yields

$$
\left(s^{2} \mathbf{M}_{u u}+s \mathbf{D}_{u u}+\mathbf{K}_{u u}^{E P *}\right) \tilde{\mathbf{u}}(s)+s \tilde{G}(s) \overline{\mathbf{K}}_{u u}^{V} \tilde{\mathbf{u}}(s)=\tilde{\mathbf{F}}(s) .
$$

Substituting the material modulus function representation in Eq. (111) into (113) yields

$$
\left(s^{2} \mathbf{M}_{u u}+s \mathbf{D}_{u u}+\mathbf{K}_{u u}^{E P *}\right) \tilde{\mathbf{u}}(s)+G_{0} \overline{\mathbf{K}}_{u u}^{V}\left(1+\sum_{i=1}^{n} \frac{\Delta_{i} s}{s+\Omega_{i}}\right) \tilde{\mathbf{u}}(s)=\tilde{\mathbf{F}}(s) .
$$

Then, introducing a set of $n$ series of anelastic (or internal, dissipation) $\tilde{\mathbf{u}}_{i}^{A}(s)(i=1, \ldots, n)$ variables, for each series, one can define the relationship

$$
\tilde{\mathbf{u}}(s)-\tilde{\mathbf{u}}_{i}^{A}(s)=\frac{s}{s+\Omega_{i}} \tilde{\mathbf{u}}(s) .
$$

Substituting Eq. (115) into (114), and considering the dissipative behavior of the anelastic DoFs given from Eq. (115) as

$$
\tilde{\mathbf{u}}_{i}^{A}(s)=\frac{\Omega_{i}}{s+\Omega_{i}} \tilde{\mathbf{u}}(s),
$$

after some algebra (see Refs. 68 and 96 for details) the time-dependent behavior of the elastic-anelastic augmented piezo-visco-elastic coupled system, is recovered through the inverse Laplace transform as

$$
\begin{gathered}
\mathbf{M}_{u u} \ddot{\mathbf{u}}(\mathbf{t})+\mathbf{D}_{u u} \dot{\overline{\mathbf{u}}}(t)+\left(\mathbf{K}_{u u}^{E P *}+\mathbf{K}_{u u}^{V \infty}\right) \overline{\mathbf{u}}(t)-\mathbf{K}_{u u}^{V 0} \sum_{i=1}^{n} \Delta_{i} \overline{\mathbf{u}}_{i}^{A}(t)=\mathbf{F}(t), \\
\frac{\Delta_{i}}{\Omega_{i}} \mathbf{K}_{u u}^{V 0} \dot{\mathbf{u}}_{i}^{A}(t)+\Delta_{i} \mathbf{K}_{u u}^{V 0} \overline{\mathbf{u}}_{i}^{A}(t)-\Delta_{i} \mathbf{K}_{u u}^{V 0} \overline{\mathbf{u}}(t)=\mathbf{0},
\end{gathered}
$$

where $\mathbf{K}_{u u}^{V 0}=G_{0} \overline{\mathbf{K}}_{u u}^{V}$ is the relaxed (static) stiffness matrix of the viscoelastic components and

$$
\mathbf{K}_{u u}^{V \infty}=\left(1+\sum_{i=1}^{n} \Delta_{i}\right) \mathbf{K}_{u u}^{V 0} .
$$

The augmented coupled system in Eqs. (117) and (118) might still be expressed in compact matrix form as

$$
\overline{\mathbf{M}} \ddot{\ddot{\mathbf{q}}}(t)+\overline{\mathbf{D}} \dot{\overline{\mathbf{q}}}(t)+\overline{\mathbf{K}} \overline{\mathbf{q}}(t)=\overline{\mathbf{F}}(t),
$$

where

$$
\begin{gathered}
\overline{\mathbf{M}}=\left[\begin{array}{cc}
\mathbf{M}_{u u} & \mathbf{0} \\
\mathbf{0} & \mathbf{0}
\end{array}\right], \quad \overline{\mathbf{D}}=\left[\begin{array}{cc}
\mathbf{D}_{u u} & \mathbf{0} \\
\mathbf{0} & \mathbf{D}_{A A}
\end{array}\right], \quad \overline{\mathbf{K}}=\left[\begin{array}{cc}
\mathbf{K}_{E E} & \mathbf{K}_{E A} \\
\mathbf{K}_{A E} & \mathbf{K}_{A A}
\end{array}\right], \\
\overline{\mathbf{q}}(t)=\operatorname{col}\left(\overline{\mathbf{u}}(t), \overline{\mathbf{u}}_{1}^{A}(t), \ldots, \overline{\mathbf{u}}_{n}^{A}(t)\right), \quad \overline{\mathbf{F}}(t)=\operatorname{col}(\mathbf{F}(t), \mathbf{0}, \ldots, \mathbf{0}),
\end{gathered}
$$

and

$$
\begin{gathered}
\mathbf{D}_{A A}=\operatorname{diag}\left(\frac{\Delta_{1}}{\Omega_{1}} \mathbf{K}_{u u}^{V 0}, \ldots, \frac{\Delta_{n}}{\Omega_{n}} \mathbf{K}_{u u}^{V 0}\right), \quad \mathbf{K}_{A A}=\operatorname{diag}\left(\Delta_{1} \mathbf{K}_{u u}^{V 0}, \ldots, \Delta_{n} \mathbf{K}_{u u}^{V 0}\right), \\
\mathbf{K}_{E E}=\mathbf{K}_{u u}^{E P *}+\mathbf{K}_{u u}^{V \infty}, \quad \mathbf{K}_{E A}=\left[-\Delta_{1} \mathbf{K}_{u u}^{V 0}, \ldots,-\Delta_{n} \mathbf{K}_{u u}^{V 0}\right], \quad \mathbf{K}_{A E}=\mathbf{K}_{E A}^{\mathrm{T}} .
\end{gathered}
$$

As can be seen in Eqs. (117) and (118), the main disadvantage of the ADF model is that, associated with a FE discretization, and in order to account for the frequency dependence of the viscoelastic material, since 
it adds auxiliary internal DoFs, which for each ADF series must be equal to the number of elastic DoFs, it leads to large systems. However, model reduction techniques might be utilized in order to reduce the size of the system. As suggested by Trindade et al. ${ }^{50}$ the matrices corresponding to the anelastic (dissipative) DoFs might be reduced and diagonalized to reduce the computational cost. Considering a linear coordinate transformation $\overline{\mathbf{u}}_{i}^{A}(t)=\boldsymbol{\Psi}_{A} \hat{\mathbf{u}}_{i}^{A}(t)$, where $\boldsymbol{\Lambda}_{A}=\boldsymbol{\Psi}_{A}^{\mathrm{T}} \mathbf{K}_{u u}^{V 0} \boldsymbol{\Psi}_{A}$ is a diagonal matrix composed by the non-zero eigenvalues of $\mathbf{K}_{u u}^{V 0}$ and $\boldsymbol{\Psi}_{A}$ is the correspondent matrix of normalized eigenvectors, such that $\boldsymbol{\Psi}_{A}^{\mathrm{T}} \boldsymbol{\Psi}_{A}=\mathbf{I}$, matrices $\mathbf{D}_{A A}, \mathbf{K}_{A A}$ and $\mathbf{K}_{A E}$, and the vector of DoFs $\overline{\mathbf{q}}(t)$, might alternatively be modified to

$$
\begin{gathered}
\mathbf{D}_{A A}=\operatorname{diag}\left(\frac{\Delta_{1}}{\Omega_{1}} \boldsymbol{\Lambda}_{A}, \ldots, \frac{\Delta_{n}}{\Omega_{n}} \boldsymbol{\Lambda}_{A}\right), \mathbf{K}_{A A}=\operatorname{diag}\left(\Delta_{1} \boldsymbol{\Lambda}_{A}, \ldots, \Delta_{n} \boldsymbol{\Lambda}_{A}\right), \\
\mathbf{K}_{E A}=\left[-\Delta_{1} \mathbf{K}_{u u}^{V 0} \boldsymbol{\Psi}_{A}, \ldots,-\Delta_{n} \mathbf{K}_{u u}^{V 0} \boldsymbol{\Psi}_{A}\right], \overline{\mathbf{q}}(t)=\operatorname{col}\left(\overline{\mathbf{u}}(t), \hat{\mathbf{u}}_{1}^{A}(t), \ldots, \hat{\mathbf{u}}_{n}^{A}(t)\right) .
\end{gathered}
$$

The advantages of the alternative (transformed) representation are that in the case where only some part of the structure is covered with viscoelastic layers only some FEs have viscoelastic components and $\mathbf{K}_{u u}^{V 0}$ can have several rows and columns of zeros, which in turn leads to some zero eigenvalues. Thus, the size of $\hat{\mathbf{u}}_{i}^{A}(t)$ can be substantially smaller than that of $\overline{\mathbf{u}}_{i}^{A}(t)$. Furthermore, one may notice from $\overline{\mathbf{M}}$ that the anelastic DoFs have no inertia and therefore the global mass matrix $\overline{\mathbf{M}}$ is singular and is not positivedefinite. However, the singularity of the mass matrix can be overcome if instead of solving the second-order system in Eq. (120) one considers a state-space representation with an adequate design of the state variables. Moreover, the number of flexible modes is kept the same and the dissipative modes, which correspond to the internal relaxations of the viscoelastic material, are overdamped with low observability. The ADF model represents a good alternative to accurately model the damping behavior of the viscoelastic materials. It is easily implemented at the FE level, yields good trade-off between accuracy and complexity and allows transient analysis.

\section{Active Control of Vibration}

\section{A. State Space Design}

The state-space approach is the basis of the modern control theories and is strongly recommended in the design and analysis of control systems with a great amount of inputs and outputs. In this method, dynamic systems are described by a set of first-order differential equations in variables called the state. See related textbooks in Refs. 116 and 117.

To apply the augmented elastic-anelastic piezo-visco-elastic coupled FE model in control design, the system in Eq. (120) is transformed into a state-space form. Therefore, in order to overcome the singularity of the mass matrix the state-space vector $\mathbf{x}(t)$ is chosen as

$$
\mathbf{x}(t)=\left\{\begin{array}{c}
\overline{\mathbf{q}}(t) \\
\dot{\overline{\mathbf{u}}}(t)
\end{array}\right\},
$$

where the chosen state variables are the augmented vector $\overline{\mathbf{q}}(t)$, composed by the mechanical (elastic) DoFs vector $\overline{\mathbf{u}}(t)$ and a reduced set of anelastic transformed coordinates vectors $\hat{\mathbf{u}}_{i}^{A}(t)$, and the the time derivative of the mechanical DoFs vector $\dot{\overline{\mathbf{u}}}(t)$. It is worthy to mention that the time derivatives of $\hat{\mathbf{u}}_{i}^{A}(t)$ are not considered here since these variables are massless. Thus, the coupled system in Eq. (120) and the sensing Eq. (109) can be expressed in terms of the state variables vector $\mathbf{x}(t)$, yielding

$$
\begin{gathered}
\dot{\mathbf{x}}(t)=\mathbf{A x}(t)+\mathbf{B}_{\phi} \mathbf{u}_{\phi}(t)+\mathbf{B}_{u} \mathbf{u}_{u}(t), \\
\mathbf{y}(t)=\mathbf{C x}(t),
\end{gathered}
$$

where $\mathbf{A}$ is the system matrix, $\mathbf{B}_{u}$ and $\mathbf{B}_{\phi}$ are the mechanical and electrical input matrices associated with the mechanical and electrical loads, $\mathbf{C}$ is the output matrix, $\mathbf{u}_{u}(t)$ and $\mathbf{u}_{\phi}(t)$ are the mechanical and electrical input vectors and $\mathbf{y}(t)$ is the output vector, given by

$$
\mathbf{A}=\left[\begin{array}{ccc}
\mathbf{0} & \mathbf{0} & \mathbf{I} \\
-\mathbf{D}_{A A}^{-1} \mathbf{K}_{A E} & -\mathbf{D}_{A A}^{-1} \mathbf{K}_{A A} & \mathbf{0} \\
-\mathbf{M}_{u u}^{-1} \mathbf{K}_{E E} & -\mathbf{M}_{u u}^{-1} \mathbf{K}_{E A} & -\mathbf{M}_{u u}^{-1} \mathbf{D}_{u u}
\end{array}\right], \quad \mathbf{B}_{u}=\left[\begin{array}{c}
\mathbf{0} \\
\mathbf{0} \\
\mathbf{M}_{u u}^{-1}
\end{array}\right], \quad \mathbf{B}_{\phi}=\left[\begin{array}{c}
\mathbf{0} \\
\mathbf{0} \\
-\mathbf{M}_{u u}^{-1} \mathbf{K}_{u \phi \mathrm{a}}
\end{array}\right]
$$




$$
\mathbf{C}=\left[\begin{array}{ccc}
-\mathbf{K}_{\phi \phi \mathbf{s}}^{-1} \mathbf{K}_{\phi u s} & \mathbf{0} & \mathbf{0} \\
\mathbf{0} & \mathbf{0} & -\mathbf{K}_{\phi \phi \mathbf{s}}^{-1} \mathbf{K}_{\phi u s} \\
\mathbf{I} & \mathbf{0} & \mathbf{0} \\
\mathbf{0} & \mathbf{0} & \mathbf{I}
\end{array}\right], \quad \mathbf{y}(t)=\left\{\begin{array}{c}
\bar{\phi}_{\mathrm{s}}(t) \\
\dot{\bar{\phi}}_{\mathrm{s}}(t) \\
\overline{\mathbf{u}}(t) \\
\dot{\mathbf{u}}(t)
\end{array}\right\}, \quad \mathbf{u}_{u}(t)=\mathbf{F}_{u}(t), \quad \mathbf{u}_{\phi}(t)=\overline{\boldsymbol{\phi}}_{\mathrm{a}}(t)
$$

The coupled piezo-visco-elastic state-space system in Eqs. (125) and (126) can therefore be used for the design and simulation of hybrid damping treatments in shells. It is however worth to mention that, as presented by Vasques and Rodrigues, ${ }^{42}$ the signal induced by the piezoelectric sensors should be calculated from an average of the electrical DoFs due to the "finite" electrical separation of the electrodes of the different elements (i.e., continuity of the electric potential difference between adjacent elements has not been enforced) and considerations regarding the equipotential area of the electrodes should be taken into account.

One major disadvantage in using internal variables models such as the ADF to model the damping introduced by the viscoelastic materials is the creation of additional dissipation coordinates. Even with a modal reduction of the DoFs of the non viscoelastic elements the order of the system quickly increases as the number of series of dissipation ADF parameters used in the summation is increased. This size is determined by the number of series of parameters necessary for an accurate curve fitting of the frequency-dependent complex shear modulus. ${ }^{68}$ Larger order makes control design more difficult, especially when these states are non-physical and can not be directly sensed. ${ }^{118}$ It is therefore advantageous to look at model reduction to reduce the system's size. As suggested by Trindade et al. ${ }^{50}$ the matrices corresponding to the internal DoFs might be reduced and diagonalized through a projection in a suitable reduced modal base. Thus, through an adequate coordinate transformation based on the eigenvalues and eigenvectors of $\mathbf{K}_{u u}^{V 0}$ and elimination of the nil eigenvalues, the size of the problem can be substantially reduced. Furthermore, truncated complex statespace modal models might also be used in order to reduce the system size even further. The reader is referred, for example, to Trindade et al., ${ }^{50}$ Vasques and Rodrigues, ${ }^{96}$ Friswell and Inman ${ }^{119}$ and Park et al. ${ }^{120}$ for further details about state-space design and model reduction techniques concerning viscoelastically damped structural systems. For further details regarding control strategies the reader is referred, for example, to the works of Vasques and Rodrigues. ${ }^{88,91,96}$

\section{B. Quantification of Damping Mechanisms}

Hybrid damping treatments attenuate vibrations and sound radiation through different damping mechanisms. In order to optimize the design of arbitrary hybrid active-passive damping treatments, where the damping layers are arbitrarily stacked and mounted on the host structure, it is essential to understand their phenomenological behavior and to be able to identify and quantify the efficiency of the different damping mechanisms. This treatments comprise on the one hand the damping effects due to the internal molecular interactions that occur during deformation in general, and vibration in particular, of the viscoelastic materials, which give rise to macroscopic properties such as stiffness and energy dissipation during cyclic deformation, and on the other hand the effects due to the piezoelectric actuation which applies forces and moments on the structure. In order to do that, an approach based upon an energetic balance of the global kinetic or strain energies is proposed.

Thus, assuming a general closed-loop control system (with a certain control strategy) applied in conjunction with arbitrary hybrid damping treatments to reduce the vibration and/or noise radiation of a general structural system, the resultant hybrid damping mechanism basically represents the net interaction and contribution of the following somewhat different but complemental three damping mechanisms (see Fig. 4):

- A passive mechanism, where the system is assumed in open-loop and where only the damping mainly due to the shearing of the viscoelastic layers (constrained or not) alone is considered;

- An active mechanism, which decreases the total input power into the structure due to the forces applied by the active piezoelectric constraining layer through the viscoelastic layer (which assumes a perfect transmissibility);

- A coupled mechanism, which represents the energy dissipation effects of the increase of shearing in the viscoelastic layer due to the convenient motion (actuation) of the active piezoelectric constraining layer. 


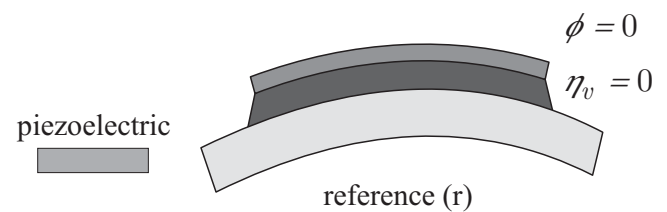

viscoelastic

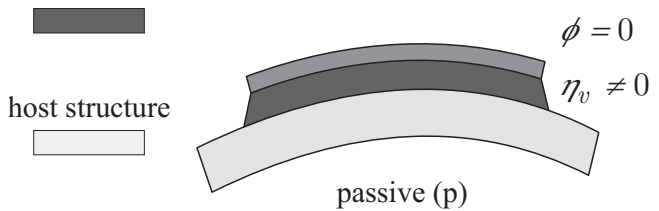

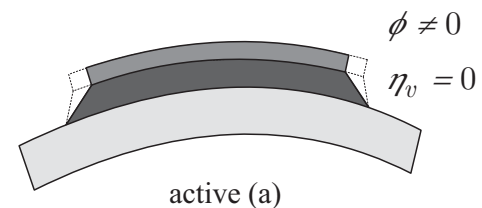

active (a)

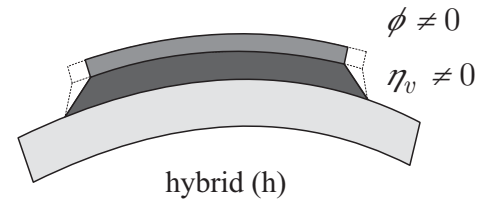

Figure 4. Schematic of the methodology to individually quantify the loss coefficients of the damping mechanisms.

Thus, based on the previous definitions, and neglecting other general sources of damping (e.g., the material damping of the elastic part of the structural system, air-based damping, energy dissipation in the supports, etc.), which were assumed to be proportional to the velocity and were considered through the viscous proportional damping model in the global FE model, a normalized loss coefficient quantifying the net damping achieved with the hybrid mechanism, $\eta_{h}$, is given as

$$
\eta_{h}=\eta_{p}+\eta_{a}+\eta_{c}
$$

where $\eta_{p}, \eta_{a}$ and $\eta_{c}$ are normalized loss coefficients that quantify the energy dissipated by the passive, active and coupled mechanisms. The hybrid, passive and active coefficients are defined as

$$
\eta_{h}=\frac{E_{r}-E_{h}}{E_{r}}, \quad \eta_{p}=\frac{E_{r}-E_{p}}{E_{r}}, \quad \eta_{a}=\frac{E_{r}-E_{a}}{E_{r}},
$$

where $E_{r}$ is the reference (kinetic or strain) energy of the structural system and $E_{h}, E_{p}$ and $E_{a}$ are the hybrid, passive and active energies (kinetic or strain) determined by setting the electric potential difference $\phi$ on or off (open- or closed-loop system) and by assuming or not the loss factor of the viscoelastic material $\eta_{v}$ equal to zero (i.e., a perfect transmissibility of the efforts from the piezoelectric patch to the host structure is considered) as depicted in Fig. 4. Since the loss coefficients in Eqs. (129) are in fact the ones that we can determine, at the simulation level, from the outputs of the system, the loss coefficient of the coupled mechanism can be determined from the others as

$$
\eta_{c}=\eta_{h}-\eta_{p}+\eta_{a}
$$

The latter loss coefficient is of great importance since it allows to infer about the advantages of hybrid damping configurations when compared with pure active or passive ones. Furthermore, when the hybrid treatments are incorrectly designed, the active mechanism might have a reduced importance since the viscoelastic layer usually reduces the transmissibility of efforts to the host structure. In fact, usually the transmissibility (stiffness) increases with frequency. Thus, in order to dissipate energy also at low frequencies, the aim would be to actively increase the shearing in the viscoelastic layer. However, due to the loss factor behavior of viscoelastic materials (usually smaller at low frequencies), the treatment is usually more efficient for frequencies with higher loss factors, if we have also significant shearing strains. (The energy released is related with the loss factor times the shearing strain.) Regarding the "pure" active treatments, the transmissibility of efforts to the host structure is higher, since the patch is bonded to the structure, and the aim is actively reducing the input power coming into the structure. Hence, this set of indices provides an efficient and straightforward means to design hybrid damping treatments since the contributions and trade-off between the different damping "ingredients" are fully known and quantified.

It is still worthy to mention that, for simplicity, the present approach to quantify the damping mechanisms has been presented and discussed for a structural system with an ACLD treatment. However, as obvious, the methodology is general and can be applied to structural systems with more complex arbitrarily stacked hybrid damping treatments. 


\section{Conclusion}

Based on a fully refined mathematical model of general anisotropic shells a fully coupled piezo-viscoelastic FE model has been conceptually proposed for multilayer shells. However, for practical reasons, related with the complexity of the formulation, simplifications regarding the through-the-thickness distribution of the transverse displacement were considered and a partially refined theory was derived with additional restrictions inherent to doubly-curved orthotropic shells physics. No simplifications regarding the thinness of the shell were considered and a plane stress state was considered for the partially refined theory.

It was shown that the refined assumptions and relaxation of some of Love's classical assumptions led to a "mixed" definition of the displacement field in terms of the same generalized displacements of the FSDT and CLT, and shear stresses on the top and bottom surfaces.

The electrical potential was defined assuming negligible in-plane electric displacement field which allowed the use of effective shear stiffness parameters which account for the effects of the induced in-plane electric field components. Regarding the transverse (out-of-plane) electrical behavior, the induced transverse electric field was considered by means of a modification of the mechanical in-plane strains, which in an analogous way were modified into effective strains. Thus, the direct piezoelectric effect was condensed into the model through effective stiffness and strains definitions, and the converse counterpart was considered by the action of prescribed electric potential differences in each piezoelectric layer.

The governing equations of a generic single layer of the multilayered shell were derived with Hamilton's principle in conjunction with the "mixed" displacement field and the electric potential difference definitions. The DoFs of the resultant four-noded generic piezo-elastic single layer FE model were then "regenerated" into an equivalent eight-node 3-D formulation in terms of top and bottom translations and shear stresses, and a transverse displacement (and its derivatives) constant in the elemental volume. The through-thethickness assemblage of the "regenerated" FE model of the single layer allowed the generation of a refined multilayer FE assuring displacement and shear stress interlayer continuity. The dynamic condensation of the stress DoFs allowed the reduction of the refined multilayer piezo-elastic FE to a an equivalent representation similar in structure to the one obtained with a first-order partial layerwise theory, but considering nonlinear in-plane displacement and quadratic shear stresses definitions and also interlayer continuity and homogeneous conditions, at the top and bottom surfaces of the refined multilayer FE, of the shear stresses. It was shown that the inclusion of the viscoelastic damping effects can be considered at the global FE model level by using a Laplace transformed version of the ADF model, with the drawback of increasing the size of the problem.

Last but not the least, the equations of motion were cast in a state-space form suitable for active control aplications/simulations, alternatives to reduce the size of the state-space piezo-visco-elastic coupled system were outlined and it was shown that a straightforward methodology using only the outputs of the statespace system can be derived to individually quantify the different damping mechanisms of arbitrary hybrid damping treatments.

The resultant partially refined piezo-visco-elastic coupled FE model can therefore be used to model shells with segmented hybrid (active-passive) damping treatments and used to design damping treatments for vibration and/or sound radiation suppression.

\section{Appendix}

\section{A. Strain-Displacement and Equilibrium Equations in Orthogonal Curvilinear Coordinates}

Taking into account that $H_{z}=1$, from the equations of 3-D theory of elasticity, the strain components of the shell layer are defined as a function of displacements by Sokolnikoff [124, pp. 177-184] as

$$
\begin{aligned}
\varepsilon_{\alpha \alpha} & =\frac{1}{H_{\alpha}} \frac{\partial u}{\partial \alpha}+\frac{1}{H_{\alpha} H_{\beta}} \frac{\partial H_{\alpha}}{\partial \beta} v+\frac{1}{H_{\alpha}} \frac{\partial H_{\alpha}}{\partial z} w, & \varepsilon_{\beta \beta} & =\frac{1}{H_{\alpha} H_{\beta}} \frac{\partial H_{\beta}}{\partial \alpha} u+\frac{1}{H_{\beta}} \frac{\partial v}{\partial \beta}+\frac{1}{H_{\beta}} \frac{\partial H_{\beta}}{\partial z} w, \\
\varepsilon_{z z} & =\frac{\partial w}{\partial z}, & \varepsilon_{\beta z} & =H_{\beta} \frac{\partial}{\partial z}\left(\frac{v}{H_{\beta}}\right)+\frac{1}{H_{\beta}} \frac{\partial w}{\partial \beta}, \\
\varepsilon_{z \alpha} & =H_{\alpha} \frac{\partial}{\partial z}\left(\frac{u}{H_{\alpha}}\right)+\frac{1}{H_{\alpha}} \frac{\partial w}{\partial \alpha}, & \varepsilon_{\alpha \beta} & =\frac{H_{\alpha}}{H_{\beta}} \frac{\partial}{\partial \beta}\left(\frac{u}{H_{\alpha}}\right)+\frac{H_{\beta}}{H_{\alpha}} \frac{\partial}{\partial \alpha}\left(\frac{v}{H_{\beta}}\right),
\end{aligned}
$$

where $u=u(\alpha, \beta, z), v=v(\alpha, \beta, z)$ and $w=w(\alpha, \beta, z)$ are the displacement components of an arbitrary point of the shell in the directions of the tangents to the coordinate lines $(\alpha, \beta, z)$, respectively. The equi- 
librium equations of a differential element of the body of the shell layer in the tri-orthogonal system of curvilinear coordinates ${ }^{124}$ is represented by the partial differential equations

$$
\begin{array}{r}
\frac{\partial}{\partial \alpha}\left(H_{\beta} \sigma_{\alpha \alpha}\right)+\frac{\partial}{\partial \beta}\left(H_{\alpha} \sigma_{\alpha \beta}\right)+\frac{\partial}{\partial z}\left(H_{\alpha} H_{\beta} \sigma_{z \alpha}\right)-\frac{\partial H_{\beta}}{\partial \alpha} \sigma_{\beta \beta}-\frac{\partial H_{\alpha}}{\partial \beta} \sigma_{\alpha \beta}+H_{\beta} \frac{\partial H_{\alpha}}{\partial z} \sigma_{z \alpha}+H_{\alpha} H_{\beta} P_{\alpha}=0, \\
\frac{\partial}{\partial \beta}\left(H_{\alpha} \sigma_{\beta \beta}\right)+\frac{\partial}{\partial \alpha}\left(H_{\beta} \sigma_{\alpha \beta}\right)+\frac{\partial}{\partial z}\left(H_{\alpha} H_{\beta} \sigma_{\beta z}\right)-\frac{\partial H_{\alpha}}{\partial \beta} \sigma_{\alpha \alpha}-\frac{\partial H_{\beta}}{\partial \alpha} \sigma_{\alpha \beta}+H_{\alpha} \frac{\partial H_{\beta}}{\partial z} \sigma_{z \beta}+H_{\alpha} H_{\beta} P_{\beta}=0, \\
\frac{\partial}{\partial z}\left(H_{\alpha} H_{\beta} \sigma_{z z}\right)+\frac{\partial}{\partial \alpha}\left(H_{\beta} \sigma_{z \alpha}\right)+\frac{\partial}{\partial \beta}\left(H_{\alpha} \sigma_{\beta z}\right)-H_{\beta} \frac{\partial H_{\alpha}}{\partial z} \sigma_{\alpha \alpha}-H_{\alpha} \frac{\partial H_{\beta}}{\partial z} \sigma_{\beta \beta}+H_{\alpha} H_{\beta} P_{z}=0,
\end{array}
$$

where $P_{\alpha}=P_{\alpha}(\alpha, \beta, z), P_{\beta}=P_{\beta}(\alpha, \beta, z)$ and $P_{z}=P_{z}(\alpha, \beta, z)$ are the corresponding projections of the volumetric force in the direction of the tangents to the shell curvilinear coordinate system.

\section{B. First Order Shear Deformation Theory (FSDT) of Anisotropic Shells}

According to the Love's first approximation assumptions for thin shells (the so-called classical Kirchhoff-Love theory of shells), the following strain and stress definitions are derived for anisotropic shells, by relaxing in part the so-called Kirchhoff's hypothesis that normals to the undeformed middle surface remain straight and normal to the deformed middle surface and suffer no extension (see [4, Sec. 1.3]). Here it is considered that normals before deformation remain straight but not necessarily normal after deformation, which basically relaxes the condition of nil out-of-plane shear strains. That theory is known as FSDT or Reissner-Mindlin theory applied to shells.

Consistent with the assumptions of a moderately thick shell theory, the displacement components are

$$
\begin{aligned}
u^{*}(\alpha, \beta, z) & =u_{0}(\alpha, \beta)+z \theta_{\alpha}(\alpha, \beta), \\
v^{*}(\alpha, \beta, z) & =v_{0}(\alpha, \beta)+z \theta_{\beta}(\alpha, \beta), \\
w^{*}(\alpha, \beta, z) & =w_{0}(\alpha, \beta),
\end{aligned}
$$

where $u_{0}=u_{0}(\alpha, \beta), v_{0}=v_{0}(\alpha, \beta)$ and $w_{0}=w_{0}(\alpha, \beta)$ are the tangential and transverse displacements referred to a point on the middle surface, respectively, and $\theta_{\alpha}=\theta_{\alpha}(\alpha, \beta)$ and $\theta_{\beta}=\theta_{\beta}(\alpha, \beta)$ are the rotations of a normal to the reference middle surface.

Thus, taken into account the strain-displacement equations of the 3-D elasticity in orthogonal curvilinear coordinates in Eqs. (A1), as proposed by Byrne, Flügge, Goldenveizer, Lur'ye and Novozhilov between the 1940s and 1960s (cf. [4, Sec. 1.4]), and in a similar form to what has been presented, for example, by Reddy [6, Sec. 8.2.3] or Leissa [4, Sec. 1.4.1], the in-plane strain definitions are given as,

$$
\begin{aligned}
& \varepsilon_{\alpha \alpha}^{*}(\alpha, \beta, z)=z_{\alpha}^{(0)} \varepsilon_{\alpha \alpha}^{*(0)}(\alpha, \beta)+z_{\alpha}^{(1)} \varepsilon_{\alpha \alpha}^{*(1)}(\alpha, \beta), \\
& \varepsilon_{\beta \beta}^{*}(\alpha, \beta, z)=z_{\beta}^{(0)} \varepsilon_{\beta \beta}^{*(0)}(\alpha, \beta)+z_{\beta}^{(1)} \varepsilon_{\beta \beta}^{*(1)}(\alpha, \beta), \\
& \varepsilon_{\alpha \beta}^{*}(\alpha, \beta, z)=z_{\alpha \beta}^{(0)} \varepsilon_{\alpha \beta}^{*(0)}(\alpha, \beta)+z_{\alpha \beta}^{(1)} \varepsilon_{\alpha \beta}^{*(1)}(\alpha, \beta),
\end{aligned}
$$

where (cf. $[4$, Sec. 1.4.1])

$$
\begin{gathered}
z_{\alpha}^{(i)}=\frac{z^{i}}{\left(1+z / R_{\alpha}\right)}, \quad z_{\beta}^{(i)}=\frac{z^{i}}{\left(1+z / R_{\beta}\right)} \\
z_{\alpha \beta}^{(0)}=z_{\alpha}^{(0)} z_{\beta}^{(0)}\left(1-\frac{z^{2}}{R_{\alpha} R_{\beta}}\right), \quad z_{\alpha \beta}^{(1)}=z_{\alpha}^{(0)} z_{\beta}^{(0)} z\left(1+\frac{z}{2 R_{\alpha}}+\frac{z}{2 R_{\beta}}\right),
\end{gathered}
$$

with $i=0,1$ and

$$
\begin{aligned}
& \varepsilon_{\alpha \alpha}^{*(0)}(\alpha, \beta)=\frac{1}{A_{\alpha}} \frac{\partial u_{0}}{\partial \alpha}+\frac{v_{0}}{A_{\alpha} A_{\beta}} \frac{\partial A_{\alpha}}{\partial \beta}+\frac{w_{0}}{R_{\alpha}}, \\
& \varepsilon_{\beta \beta}^{*(0)}(\alpha, \beta)=\frac{1}{A_{\beta}} \frac{\partial v_{0}}{\partial \beta}+\frac{u_{0}}{A_{\alpha} A_{\beta}} \frac{\partial A_{\beta}}{\partial \alpha}+\frac{w_{0}}{R_{\beta}}, \\
& \varepsilon_{\alpha \beta}^{*(0)}(\alpha, \beta)=\frac{A_{\alpha}}{A_{\beta}} \frac{\partial}{\partial \beta}\left(\frac{u_{0}}{A_{\alpha}}\right)+\frac{A_{\beta}}{A_{\alpha}} \frac{\partial}{\partial \alpha}\left(\frac{v_{0}}{A_{\beta}}\right),
\end{aligned}
$$




$$
\begin{aligned}
\varepsilon_{\alpha \alpha}^{*(1)}(\alpha, \beta) & =\frac{1}{A_{\alpha}} \frac{\partial \theta_{\alpha}}{\partial \alpha}+\frac{1}{A_{\alpha} A_{\beta}} \frac{\partial A_{\alpha}}{\partial \beta} \theta_{\beta} \\
\varepsilon_{\beta \beta}^{*(1)}(\alpha, \beta) & =\frac{1}{A_{\beta}} \frac{\partial \theta_{\beta}}{\partial \beta}+\frac{1}{A_{\alpha} A_{\beta}} \frac{\partial A_{\beta}}{\partial \alpha} \theta_{\alpha} \\
\varepsilon_{\alpha \beta}^{*(1)}(\alpha, \beta) & =\frac{A_{\alpha}}{A_{\beta}} \frac{\partial}{\partial \beta}\left(\frac{\theta_{\alpha}}{A_{\alpha}}\right)+\frac{A_{\beta}}{A_{\alpha}} \frac{\partial}{\partial \alpha}\left(\frac{\theta_{\beta}}{A_{\beta}}\right) \\
& +\frac{1}{R_{\alpha}}\left(\frac{1}{A_{\beta}} \frac{\partial u_{0}}{\partial \beta}-\frac{1}{A_{\alpha} A_{\beta}} \frac{\partial A_{\beta}}{\partial \alpha} v_{0}\right)+\frac{1}{R_{\beta}}\left(\frac{1}{A_{\alpha}} \frac{\partial v_{0}}{\partial \alpha}-\frac{1}{A_{\alpha} A_{\beta}} \frac{\partial A_{\alpha}}{\partial \beta} u_{0}\right) .
\end{aligned}
$$

Regarding the previous strain definitions it is worthy to mention that the zero-order terms $\varepsilon_{\alpha \alpha}^{*(0)}$, $\varepsilon_{\beta \beta}^{*(0)}$ and $\varepsilon_{\alpha \beta}^{*(0)}$ represent the normal (membrane) and shearing strains of the reference surface, respectively, and the first order terms $\varepsilon_{\alpha \alpha}^{*(1)}, \varepsilon_{\beta \beta}^{*(1)}$ and $\varepsilon_{\alpha \beta}^{*(1)}$ represent the linearly distributed bending components of strain and the torsion of the reference surface during deformation.

Considering the mechanical counterpart of the anisotropic (general orthotropic) plane-stress constitutive behavior presented in Eq. (C15), the in-plane stresses are given as

$$
\begin{aligned}
& \sigma_{\alpha \alpha}^{*}(\alpha, \beta, z)=\bar{c}_{11}^{*} \varepsilon_{\alpha \alpha}^{*}(\alpha, \beta, z)+\bar{c}_{12}^{*} \varepsilon_{\beta \beta}^{*}(\alpha, \beta, z)+\bar{c}_{16}^{*} \varepsilon_{\alpha \beta}^{*}(\alpha, \beta, z)=\sigma_{\alpha \alpha}^{*(0)}(\alpha, \beta, z)+\sigma_{\alpha \alpha}^{*(1)}(\alpha, \beta, z), \\
& \sigma_{\beta \beta}^{*}(\alpha, \beta, z)=\bar{c}_{12}^{*} \varepsilon_{\alpha \alpha}^{*}(\alpha, \beta, z)+\bar{c}_{22}^{*} \varepsilon_{\beta \beta}^{*}(\alpha, \beta, z)+\bar{c}_{26}^{*} \varepsilon_{\alpha \beta}^{*}(\alpha, \beta, z)=\sigma_{\beta \beta}^{*(0)}(\alpha, \beta, z)+\sigma_{\beta \beta}^{*(1)}(\alpha, \beta, z), \\
& \sigma_{\alpha \beta}^{*}(\alpha, \beta, z)=\bar{c}_{16}^{*} \varepsilon_{\alpha \alpha}^{*}(\alpha, \beta, z)+\bar{c}_{26}^{*} \varepsilon_{\beta \beta}^{*}(\alpha, \beta, z)+\bar{c}_{66}^{*} \varepsilon_{\alpha \beta}^{*}(\alpha, \beta, z)=\sigma_{\alpha \beta}^{*(0)}(\alpha, \beta, z)+\sigma_{\alpha \beta}^{*(1)}(\alpha, \beta, z),
\end{aligned}
$$

where in a similar way to the zero-order and first-order strain definitions, Eqs. (B5) are splitted into the zero-order and first-order stress components, where, for example, for the first Eq. of (B5)

$$
\begin{aligned}
& \sigma_{\alpha \alpha}^{*(0)}(\alpha, \beta, z)=z_{\alpha}^{(0)} \bar{c}_{11}^{*} \varepsilon_{\alpha \alpha}^{*(0)}(\alpha, \beta)+z_{\beta}^{(0)} \bar{c}_{12}^{*} \varepsilon_{\beta \beta}^{*(0)}(\alpha, \beta)+z_{\alpha \beta}^{(0)} \bar{c}_{16}^{*} \varepsilon_{\alpha \beta}^{*(0)}(\alpha, \beta), \\
& \sigma_{\alpha \alpha}^{*(1)}(\alpha, \beta, z)=z_{\alpha}^{(1)} \bar{c}_{11}^{*} \varepsilon_{\alpha \alpha}^{*(1)}(\alpha, \beta)+z_{\beta}^{(1)} \bar{c}_{12}^{*} \varepsilon_{\beta \beta}^{*(1)}(\alpha, \beta)+z_{\alpha \beta}^{(1)} \bar{c}_{16}^{*} \varepsilon_{\alpha \beta}^{*(1)}(\alpha, \beta) .
\end{aligned}
$$

Similar relations hold for the second and third Eqs. of (B5) which for the sake of brevity are not presented here.

\section{Piezoelectric Constitutive Behavior}

The linear piezoelectric constitutive equations in compact matrix notation ${ }^{104}$ are given by

$$
\begin{aligned}
\boldsymbol{\sigma} & =\mathbf{c}^{E} \boldsymbol{\varepsilon}-\mathbf{e}^{\mathrm{T}} \mathbf{E}, \\
\mathbf{D} & =\mathbf{e} \boldsymbol{\varepsilon}+\boldsymbol{\epsilon}^{S} \mathbf{E}
\end{aligned}
$$

where $\boldsymbol{\sigma}, \boldsymbol{\varepsilon}, \mathbf{E}$ and $\mathbf{D}$ are, respectively, the stress, strain, electric field and electric displacement vectors, and $\mathbf{c}^{E}, \mathbf{e}^{T}$ and $\boldsymbol{\epsilon}^{S}$ are, respectively, the elasticity (at constant electric field), transpose piezoelectric and dielectric (at constant strain) matrices appropriate for the material.

The material of piezoelectric layers is assumed to be general orthotropic, with the axes of orthotropy not necessarily parallel (arbitrary orientation of the piezoelectric shell layer) to the axes of principal curvature of the shell layer $(\alpha, \beta, z)$, and polarized in the transverse direction $z$. Furthermore, it has the symmetry properties of an orthorhombic material of the class $m m 2 .{ }^{103,104}$ Representing Eqs. (C1) and (C2), with their full matrix and vector terms in engineering notation yields

$$
\left\{\begin{array}{c}
\sigma_{\alpha \alpha} \\
\sigma_{\beta \beta} \\
\sigma_{z z} \\
\sigma_{\beta z} \\
\sigma_{z \alpha} \\
\sigma_{\alpha \beta}
\end{array}\right\}=\left[\begin{array}{cccccc}
\bar{c}_{11} & \bar{c}_{12} & \bar{c}_{13} & 0 & 0 & \bar{c}_{16} \\
\bar{c}_{12} & \bar{c}_{22} & \bar{c}_{23} & 0 & 0 & \bar{c}_{26} \\
\bar{c}_{13} & \bar{c}_{23} & \bar{c}_{33} & 0 & 0 & \bar{c}_{36} \\
0 & 0 & 0 & \bar{c}_{44} & \bar{c}_{45} & 0 \\
0 & 0 & 0 & \bar{c}_{45} & \bar{c}_{55} & 0 \\
\bar{c}_{16} & \bar{c}_{26} & \bar{c}_{36} & 0 & 0 & \bar{c}_{66}
\end{array}\right]\left\{\begin{array}{l}
\varepsilon_{\alpha \alpha} \\
\varepsilon_{\beta \beta} \\
\varepsilon_{z z} \\
\varepsilon_{\beta z} \\
\varepsilon_{z \alpha} \\
\varepsilon_{\alpha \beta}
\end{array}\right\}-\left[\begin{array}{ccc}
0 & 0 & \bar{e}_{31} \\
0 & 0 & \bar{e}_{32} \\
0 & 0 & \bar{e}_{33} \\
\bar{e}_{14} & \bar{e}_{24} & 0 \\
\bar{e}_{15} & \bar{e}_{25} & 0 \\
0 & 0 & \bar{e}_{36}
\end{array}\right]\left\{\begin{array}{l}
E_{\alpha} \\
E_{\beta} \\
E_{z}
\end{array}\right\},
$$




$$
\left\{\begin{array}{l}
D_{\alpha} \\
D_{\beta} \\
D_{z}
\end{array}\right\}=\left[\begin{array}{cccccc}
0 & 0 & 0 & \bar{e}_{14} & \bar{e}_{15} & 0 \\
0 & 0 & 0 & \bar{e}_{24} & \bar{e}_{25} & 0 \\
\bar{e}_{31} & \bar{e}_{32} & \bar{e}_{33} & 0 & 0 & \bar{e}_{36}
\end{array}\right]\left\{\begin{array}{l}
\varepsilon_{\alpha \alpha} \\
\varepsilon_{\beta \beta} \\
\varepsilon_{z z} \\
\varepsilon_{\beta z} \\
\varepsilon_{z \alpha} \\
\varepsilon_{\alpha \beta}
\end{array}\right\}+\left[\begin{array}{ccc}
\bar{\epsilon}_{11} & \bar{\epsilon}_{12} & 0 \\
\bar{\epsilon}_{12} & \bar{\epsilon}_{22} & 0 \\
0 & 0 & \bar{\epsilon}_{33}
\end{array}\right]\left\{\begin{array}{l}
E_{\alpha} \\
E_{\beta} \\
E_{z}
\end{array}\right\} .
$$

For simplicity the superscripts $(\cdot)^{E}$ and $(\cdot)^{S}$ of Eqs. (C1) and (C2) have been dropped. The relationships between the problem quantities $\bar{c}_{i j}, \bar{e}_{r j}$ and $\bar{\epsilon}_{r s}(i, j=1, \ldots, 6 ; r, s=1,2,3)$ and the original material $c_{i j}$, $e_{r j}$ and $\epsilon_{r s}$ when the material system $\alpha \beta$-plane is rotated an angle $+\theta$ (rotating from $\alpha$ to $\beta$ ) around the $z$-axis are given by

$$
\begin{array}{lll}
\bar{c}_{11}=c_{11} \cos ^{4} \theta+2\left(c_{12}+2 c_{66}\right) \sin ^{2} \theta \cos ^{2} \theta+c_{22} \sin ^{4} \theta, & \bar{c}_{13}=c_{13} \cos ^{2} \theta+c_{23} \sin ^{2} \theta, \\
\bar{c}_{12}=c_{12}\left(\sin ^{4} \theta+\cos ^{4} \theta\right)+\left(c_{11}+c_{22}-4 c_{66}\right) \sin ^{2} \theta \cos ^{2} \theta, & \bar{c}_{23}=c_{13} \sin ^{2} \theta+c_{23} \cos ^{2} \theta, \\
\bar{c}_{16}=\left(c_{11}-c_{12}-2 c_{66}\right) \sin \theta \cos ^{3} \theta+\left(c_{12}-c_{22}+2 c_{66}\right) \sin ^{3} \theta \cos \theta, & \bar{c}_{36}=\left(c_{13}-c_{23}\right) \sin \theta \cos \theta, \\
\bar{c}_{22}=c_{11} \sin ^{4} \theta+2\left(c_{12}+2 c_{66}\right) \sin ^{2} \theta \cos ^{2} \theta+c_{22} \cos ^{4} \theta, & \bar{c}_{44}=c_{44} \cos ^{2} \theta+c_{55} \sin ^{2} \theta, \\
\bar{c}_{26}=\left(c_{11}-c_{12}-2 c_{66}\right) \sin ^{3} \theta \cos \theta+\left(c_{12}-c_{22}+2 c_{66}\right) \sin \theta \cos ^{3} \theta, & \bar{c}_{45}=\left(c_{55}-c_{44}\right) \sin \theta \cos \theta, \\
\bar{c}_{66}=2\left(c_{11}+c_{22}-2 c_{12}\right) \sin ^{2} \theta \cos ^{2} \theta+c_{66}\left(\sin ^{2} \theta-\cos ^{2} \theta\right)^{2}, & \bar{c}_{55}=c_{55} \cos ^{2} \theta+c_{44} \sin ^{2} \theta, \\
\bar{c}_{33}=c_{33}, &
\end{array}
$$

and

$$
\begin{array}{ll}
\bar{e}_{14}=\left(e_{15}-e_{24}\right) \sin \theta \cos \theta, & \bar{e}_{33}=e_{33}, \\
\bar{e}_{15}=e_{15} \cos ^{2} \theta+e_{24} \sin ^{2} \theta, & \bar{e}_{36}=\left(e_{31}-e_{32}\right) \sin \theta \cos \theta, \\
\bar{e}_{24}=e_{15} \sin ^{2} \theta+e_{24} \cos ^{2} \theta, & \bar{\epsilon}_{11}=\epsilon_{11} \cos ^{2} \theta+\epsilon_{22} \sin ^{2} \theta, \\
\bar{e}_{25}=\left(e_{15}-e_{24}\right) \sin \theta \cos \theta, & \bar{\epsilon}_{12}=\left(\epsilon_{11}-\epsilon_{22}\right) \sin \theta \cos \theta, \\
\bar{e}_{31}=e_{31} \cos ^{2} \theta+e_{32} \sin ^{2} \theta, & \bar{\epsilon}_{22}=\epsilon_{11} \sin ^{2} \theta+\epsilon_{22} \cos ^{2} \theta, \\
\bar{e}_{32}=e_{31} \sin ^{2} \theta+e_{32} \cos ^{2} \theta, & \bar{\epsilon}_{33}=\epsilon_{33} .
\end{array}
$$

The electrostatic equilibrium of a piezoelectric media expressed in the constitutive Eq. (C3) can be re-written has

$$
\left\{\begin{array}{l}
D_{\alpha} \\
D_{\beta} \\
D_{z}
\end{array}\right\}=\left[\begin{array}{ccc}
\bar{\epsilon}_{11} & \bar{\epsilon}_{12} & 0 \\
\bar{\epsilon}_{12} & \bar{\epsilon}_{22} & 0 \\
0 & 0 & \bar{\epsilon}_{33}
\end{array}\right]\left\{\begin{array}{l}
\hat{E}_{\alpha}-\bar{E}_{\alpha} \\
\hat{E}_{\beta}-\bar{E}_{\beta} \\
\hat{E}_{z}-\bar{E}_{z}
\end{array}\right\},
$$

where $\hat{E}_{\alpha}, \hat{E}_{\beta}$ and $\hat{E}_{z}$ are the electric field components due to the converse prescribed (externally applied) piezoelectric effects and $\bar{E}_{\alpha}, \bar{E}_{\beta}$ and $\bar{E}_{z}$ are the electric field components due to the direct (induced) piezoelectric effect given by

$$
\begin{aligned}
& \bar{E}_{\alpha}=-\frac{\bar{\epsilon}_{22}}{\bar{\epsilon}_{12}^{*}}\left(\bar{e}_{14} \varepsilon_{\beta z}+\bar{e}_{15} \varepsilon_{z \alpha}\right)+\frac{\bar{\epsilon}_{12}}{\bar{\epsilon}_{12}^{*}}\left(\bar{e}_{24} \varepsilon_{\beta z}+\bar{e}_{25} \varepsilon_{z \alpha}\right), \\
& \bar{E}_{\beta}=+\frac{\bar{\epsilon}_{12}}{\bar{\epsilon}_{12}^{*}}\left(\bar{e}_{14} \varepsilon_{\beta z}+\bar{e}_{15} \varepsilon_{z \alpha}\right)-\frac{\bar{\epsilon}_{11}}{\bar{\epsilon}_{12}^{*}}\left(\bar{e}_{24} \varepsilon_{\beta z}+\bar{e}_{25} \varepsilon_{z \alpha}\right), \\
& \bar{E}_{z}=-\frac{1}{\bar{\epsilon}_{33}}\left(\bar{e}_{31} \varepsilon_{\alpha \alpha}+\bar{e}_{32} \varepsilon_{\beta \beta}+\bar{e}_{33} \varepsilon_{z z}+\bar{e}_{36} \varepsilon_{\alpha \beta}\right),
\end{aligned}
$$

where $\bar{\epsilon}_{12}^{*}=\bar{\epsilon}_{11} \bar{\epsilon}_{22}-\left(\bar{\epsilon}_{12}\right)^{2}$.

Since in the present problem the electrodes are located on the top and bottom surfaces and only a transverse electric field $\hat{E}_{z}$ is externally applied, the conditions $D_{\alpha}=D_{\beta}=0$ hold in the shell surfaces not covered with electrodes and in contact with a medium with low permittivity, e.g., vacuum or air (see Refs. 40,42 and 45 for further details). Thus, considering the relationships in (C7) the prescribed in-plane electric field components $\hat{E}_{\alpha}$ and $\hat{E}_{\beta}$ are equal to the induced ones, and in matrix form they are determined from 
Eq. (C4),

$$
\left\{\begin{array}{l}
E_{\alpha} \\
E_{\beta}
\end{array}\right\}=-\left[\begin{array}{ll}
\bar{\epsilon}_{11} & \bar{\epsilon}_{12} \\
\bar{\epsilon}_{12} & \bar{\epsilon}_{22}
\end{array}\right]^{-1}\left[\begin{array}{llllll}
0 & 0 & 0 & \bar{e}_{14} & \bar{e}_{15} & 0 \\
0 & 0 & 0 & \bar{e}_{24} & \bar{e}_{25} & 0
\end{array}\right]\left\{\begin{array}{l}
\varepsilon_{\alpha \alpha} \\
\varepsilon_{\beta \beta} \\
\varepsilon_{z z} \\
\varepsilon_{\beta z} \\
\varepsilon_{z \alpha} \\
\varepsilon_{\alpha \beta}
\end{array}\right\} .
$$

Condensing the in-plane induced electric fields in Eq. (C3) yields

$$
\begin{aligned}
\left\{\begin{array}{c}
\sigma_{\alpha \alpha} \\
\sigma_{\beta \beta} \\
\sigma_{z z} \\
\sigma_{\beta z} \\
\sigma_{z \alpha} \\
\sigma_{\alpha \beta}
\end{array}\right\}=\left[\begin{array}{cccccc}
\bar{c}_{11} & \bar{c}_{12} & \bar{c}_{13} & 0 & 0 & \bar{c}_{16} \\
\bar{c}_{12} & \bar{c}_{22} & \bar{c}_{23} & 0 & 0 & \bar{c}_{26} \\
\bar{c}_{13} & \bar{c}_{23} & \bar{c}_{33} & 0 & 0 & \bar{c}_{36} \\
0 & 0 & 0 & \bar{c}_{44}^{\star} & \bar{c}_{45}^{\star} & 0 \\
0 & 0 & 0 & \bar{c}_{45}^{\star} & \bar{c}_{55}^{\star} & 0 \\
\bar{c}_{16} & \bar{c}_{26} & \bar{c}_{36} & 0 & 0 & \bar{c}_{66}
\end{array}\right]\left\{\begin{array}{l}
\varepsilon_{\alpha \alpha} \\
\varepsilon_{\beta \beta} \\
\varepsilon_{z z} \\
\varepsilon_{\beta z} \\
\varepsilon_{z \alpha} \\
\varepsilon_{\alpha \beta}
\end{array}\right\}-\left[\begin{array}{c}
\bar{e}_{31} \\
\bar{e}_{32} \\
\bar{e}_{33} \\
0 \\
0 \\
\bar{e}_{36}
\end{array}\right] E_{z}, \\
D_{z}=\left[\begin{array}{llllll}
\bar{e}_{31} & \bar{e}_{32} & \bar{e}_{33} & 0 & 0 & \bar{e}_{36}
\end{array}\right]\left\{\begin{array}{l}
\varepsilon_{\alpha \alpha} \\
\varepsilon_{\beta \beta} \\
\varepsilon_{z z} \\
\varepsilon_{\beta z} \\
\varepsilon_{z \alpha} \\
\varepsilon_{\alpha \beta}
\end{array}\right\}+\bar{\epsilon}_{33} E_{z},
\end{aligned}
$$

where the modified constants (or effective shear stiffness parameters) $\bar{c}_{44}^{*}, \bar{c}_{45}^{*}$ and $\bar{c}_{55}^{*}$ are given by

$$
\begin{gathered}
\bar{c}_{44}^{\star}=\bar{c}_{44}-\frac{\bar{\epsilon}_{11}\left(\bar{e}_{24}\right)^{2}-2 \bar{e}_{14} \bar{\epsilon}_{12} \bar{e}_{24}+\bar{\epsilon}_{22}\left(\bar{e}_{14}\right)^{2}}{\bar{\epsilon}_{11} \bar{\epsilon}_{22}-\left(\bar{\epsilon}_{12}\right)^{2}}, \quad \bar{c}_{55}^{\star}=\bar{c}_{55}-\frac{\bar{\epsilon}_{11}\left(\bar{e}_{25}\right)^{2}-2 \bar{e}_{15} \bar{\epsilon}_{12} \bar{e}_{25}+\bar{\epsilon}_{22}\left(\bar{e}_{15}\right)^{2}}{\bar{\epsilon}_{11} \bar{\epsilon}_{22}-\left(\bar{\epsilon}_{12}\right)^{2}} \\
\bar{c}_{45}^{\star}=\bar{c}_{45}-\frac{\bar{e}_{15} \bar{\epsilon}_{22} \bar{e}_{14}-\bar{e}_{15} \bar{\epsilon}_{12} \bar{e}_{24}-\bar{e}_{25} \bar{\epsilon}_{12} \bar{e}_{14}+\bar{e}_{25} \bar{\epsilon}_{11} \bar{e}_{24}}{\bar{\epsilon}_{11} \bar{\epsilon}_{22}-\left(\bar{\epsilon}_{12}\right)^{2}}
\end{gathered}
$$

Similar relations to Eq. (C1) can be expressed for the full strain-stress relationship,

$$
\varepsilon=\mathbf{s}^{E} \boldsymbol{\sigma}-\mathbf{d}^{\mathrm{T}} \mathbf{E}
$$

where $\mathbf{s}^{E}$ is the compliance matrix (at constant electric field) and $\mathbf{d}^{T}$ the transpose piezoelectric strain constant matrix. From Eq. (C10), the reduced form in full matrix and vector terms reads

$$
\left\{\begin{array}{c}
\varepsilon_{\alpha \alpha} \\
\varepsilon_{\beta \beta} \\
\varepsilon_{z z} \\
\varepsilon_{\beta z} \\
\varepsilon_{z \alpha} \\
\varepsilon_{\alpha \beta}
\end{array}\right\}=\left[\begin{array}{cccccc}
\bar{s}_{11} & \bar{s}_{12} & \bar{s}_{13} & 0 & 0 & \bar{s}_{16} \\
\bar{s}_{12} & \bar{s}_{22} & \bar{s}_{23} & 0 & 0 & \bar{s}_{26} \\
\bar{s}_{13} & \bar{s}_{23} & \bar{s}_{33} & 0 & 0 & \bar{s}_{36} \\
0 & 0 & 0 & \bar{s}_{44}^{\star} & \bar{s}_{45}^{\star} & 0 \\
0 & 0 & 0 & \bar{s}_{45}^{\star} & \bar{s}_{55}^{\star} & 0 \\
\bar{s}_{16} & \bar{s}_{26} & \bar{s}_{36} & 0 & 0 & \bar{s}_{66}
\end{array}\right]\left\{\begin{array}{c}
\sigma_{\alpha \alpha} \\
\sigma_{\beta \beta} \\
\sigma_{z z} \\
\sigma_{\beta z} \\
\sigma_{z \alpha} \\
\sigma_{\alpha \beta}
\end{array}\right\}-\left[\begin{array}{c}
\bar{d}_{31} \\
\bar{d}_{32} \\
\bar{d}_{33} \\
0 \\
0 \\
\bar{d}_{36}
\end{array}\right] E_{z},
$$

where the problem quantities $\bar{s}_{i j}$, with the shear effective (modified) compliance parameters, and $\bar{d}_{r j}$ might be obtained from the relationships $\mathbf{s}^{E}=\left(\mathbf{c}^{E}\right)^{-1}$, taking the matrix $\mathbf{c}^{E}$ defined in Eq. (C10), and $\mathbf{d}=\mathbf{e} \mathbf{s}^{E}$. 
If one now reduces Eqs. (C10) and (C11) to the plane-stress constitutive behavior, where $\sigma_{z z} \approx 0$, yields

$$
\begin{gathered}
\left\{\begin{array}{c}
\sigma_{\alpha \alpha} \\
\sigma_{\beta \beta} \\
\sigma_{\beta z} \\
\sigma_{z \alpha} \\
\sigma_{\alpha \beta}
\end{array}\right\}=\left[\begin{array}{ccccc}
\bar{c}_{11}^{*} & \bar{c}_{12}^{*} & 0 & 0 & \bar{c}_{16}^{*} \\
\bar{c}_{12}^{*} & \bar{c}_{22}^{*} & 0 & 0 & \bar{c}_{26}^{*} \\
0 & 0 & \bar{c}_{44}^{\star} & \bar{c}_{45}^{\star} & 0 \\
0 & 0 & \bar{c}_{45}^{\star} & \bar{c}_{55}^{\star} & 0 \\
\bar{c}_{16}^{*} & \bar{c}_{26}^{*} & 0 & 0 & \bar{c}_{66}^{*}
\end{array}\right]\left\{\begin{array}{l}
\varepsilon_{\alpha \alpha} \\
\varepsilon_{\beta \beta} \\
\varepsilon_{\beta z} \\
\varepsilon_{z \alpha} \\
\varepsilon_{\alpha \beta}
\end{array}\right\}-\left[\begin{array}{c}
\bar{e}_{31}^{*} \\
\bar{e}_{32}^{*} \\
0 \\
0 \\
\bar{e}_{36}^{*}
\end{array}\right] E_{z}, \\
D_{z}=\left[\begin{array}{lllll}
\bar{e}_{31}^{*} & \bar{e}_{32}^{*} & 0 & 0 & \bar{e}_{36}^{*}
\end{array}\right]\left\{\begin{array}{l}
\varepsilon_{\alpha \alpha} \\
\varepsilon_{\beta \beta} \\
\varepsilon_{\beta z} \\
\varepsilon_{z \alpha} \\
\varepsilon_{\alpha \beta}
\end{array}\right\}+\bar{\epsilon}_{33}^{*} E_{z}=\bar{\epsilon}_{33}^{*}\left(\hat{E}_{z}-\bar{E}_{z}\right),
\end{gathered}
$$

where the elastic stiffness constants have been modified to take the plane-stress assumption into account and are defined as

$$
\begin{array}{lll}
\bar{c}_{11}^{*}=\bar{c}_{11}-\frac{\left(\bar{c}_{13}\right)^{2}}{\bar{c}_{33}}, & \bar{c}_{22}^{*}=\bar{c}_{22}-\frac{\left(\bar{c}_{23}\right)^{2}}{\bar{c}_{33}}, & \bar{c}_{66}^{*}=\bar{c}_{66}-\frac{\left(\bar{c}_{36}\right)^{2}}{\bar{c}_{33}}, \\
\bar{c}_{12}^{*}=\bar{c}_{12}-\frac{\bar{c}_{13} \bar{c}_{23}}{\bar{c}_{33}}, & \bar{c}_{16}^{*}=\bar{c}_{16}-\frac{\bar{c}_{13} \bar{c}_{36}}{\bar{c}_{33}}, & \bar{c}_{26}^{*}=\bar{c}_{26}-\frac{\bar{c}_{23} \bar{c}_{36}}{\bar{c}_{33}},
\end{array}
$$

and $\bar{e}_{31}^{*}, \bar{e}_{32}^{*}$ and $\bar{e}_{32}^{*}$ are reduced (modified) piezoelectric plane-stress constants and $\bar{\epsilon}_{33}^{*}$ the dielectric one, given by

$$
\bar{e}_{31}^{*}=\bar{e}_{31}-\bar{e}_{33} \frac{\bar{c}_{13}}{\bar{c}_{33}}, \quad \bar{e}_{32}^{*}=\bar{e}_{32}-\bar{e}_{33} \frac{\bar{c}_{23}}{\bar{c}_{33}}, \quad \bar{e}_{36}^{*}=\bar{e}_{36}-\bar{e}_{33} \frac{\bar{c}_{36}}{\bar{c}_{33}}, \quad \bar{\epsilon}_{33}^{*}=\bar{\epsilon}_{33}+\frac{\left(\bar{e}_{33}\right)^{2}}{\bar{c}_{33}} .
$$

Similar relations to the previous equations hold for the reduced compliances and piezoelectric strain constants when the plane-stress is considered.

\section{Mixed Displacement Field and Strains}

According to the in-plane mixed displacements definition in Eqs. (41) and taking into account the shear angles definition in Eqs. (27), the displacement field can be expresses as

$$
\begin{aligned}
u(\alpha, \beta, z, t) & =\frac{u_{0}}{z_{\alpha}^{(0)}}+\frac{z_{\alpha}^{*(f)} \bar{s}_{55}^{*}}{z_{\alpha}^{(0)}}\left(\frac{\partial w_{0}}{\partial \alpha}+\theta_{\alpha}-\frac{u_{0}}{R_{\alpha}}\right)-\frac{z_{\alpha}^{*(0)}}{z_{\alpha}^{(0)}} \frac{\partial w_{0}}{\partial \alpha}+\frac{z_{\alpha}^{*(0)} \bar{s}_{55}^{*}}{z_{\alpha}^{(0)}} \bar{\tau}_{z \alpha}+\frac{z_{\alpha}^{*(1)} \bar{s}_{55}^{*}}{z_{\alpha}^{(0)} 2 h} \tilde{\tau}_{z \alpha} \\
& =z_{11}^{u} u_{0}+z_{13}^{u} \frac{\partial w_{0}}{\partial \alpha}+z_{14}^{u} \theta_{\alpha}+z_{11}^{\tau} \bar{\tau}_{z \alpha}+z_{12}^{\tau} \tilde{\tau}_{z \alpha}, \\
v(\alpha, \beta, z, t) & =\frac{v_{0}}{z_{\beta}^{(0)}}+\frac{z_{\beta}^{*(f)} \bar{s}_{44}^{*}}{z_{\beta}^{(0)}}\left(\frac{\partial w_{0}}{\partial \beta}+\theta_{\beta}-\frac{v_{0}}{R_{\beta}}\right)-\frac{z_{\beta}^{*(0)}}{z_{\beta}^{(0)}} \frac{\partial w_{0}}{\partial \beta}+\frac{z_{\beta}^{*(0)} \bar{s}_{44}^{*}}{z_{\beta}^{(0)}} \bar{\tau}_{\beta z}+\frac{z_{\beta}^{*(1)} \bar{s}_{44}^{*}}{z_{\beta}^{(0)} 2 h} \tilde{\tau}_{\beta z} \\
& =z_{22}^{u} v_{0}+z_{23}^{u} \frac{\partial w_{0}}{\partial \beta}+z_{25}^{u} \theta_{\beta}+z_{23}^{\tau} \bar{\tau}_{\beta z}+z_{24}^{\tau} \tilde{\tau}_{\beta z},
\end{aligned}
$$

where the $z_{i j}^{u}=z_{i j}^{u}(z)$ and $z_{i j}^{u}=z_{i r}^{\sigma}(z)$ coefficients are given by

$$
\begin{aligned}
& z_{11}^{u}=\frac{1}{z_{\alpha}^{(0)}}-\frac{z_{\alpha}^{*(f)} \bar{s}_{55}^{*}}{z_{\alpha}^{(0)} R_{\alpha}}, \quad z_{13}^{u}=\frac{z_{\alpha}^{*(f)} \bar{s}_{55}^{*}}{z_{\alpha}^{(0)}}-\frac{z_{\alpha}^{*(0)}}{z_{\alpha}^{(0)}}, \quad z_{14}^{u}=\frac{z_{\alpha}^{*(f)} \bar{s}_{55}^{*}}{z_{\alpha}^{(0)}}, \\
& z_{22}^{u}=\frac{1}{z_{\beta}^{(0)}}-\frac{z_{\beta}^{*(f)} \bar{s}_{44}^{*}}{z_{\beta}^{(0)} R_{\beta}}, \quad z_{23}^{u}=\frac{z_{\beta}^{*(f)} \bar{s}_{44}^{*}}{z_{\beta}^{(0)}}-\frac{z_{\beta}^{*(0)}}{z_{\beta}^{(0)}}, \quad z_{25}^{u}=\frac{z_{\beta}^{*(f)} \bar{s}_{44}^{*}}{z_{\beta}^{(0)}}, \\
& z_{11}^{\tau}=\frac{z_{\alpha}^{*(0)} \bar{s}_{55}^{*}}{z_{\alpha}^{(0)}}, \quad z_{12}^{\tau}=\frac{z_{\alpha}^{*(1)}}{z_{\alpha}^{(0)}} \frac{\bar{s}_{55}^{*}}{2 h}, \quad z_{23}^{\tau}=\frac{z_{\beta}^{*(0)} \bar{s}_{44}^{*}}{z_{\beta}^{(0)}}, \quad z_{24}^{\tau}=\frac{z_{\beta}^{*(1)}}{z_{\beta}^{(0)}} \frac{\bar{s}_{44}^{*}}{2 h} .
\end{aligned}
$$


It is worthy to mention that these terms are functions of $z$ and incorporate elastic constants of the material and geometric variables of the shell layer. by

The non-zero coefficients of matrices $\mathbf{z}^{\varepsilon u}(z)$ and $\mathbf{z}^{\varepsilon \tau}(z)$ used to define the strain field in Eq. (47) are given

$$
\begin{gathered}
z_{11}^{\varepsilon u}=z_{\alpha}^{(0)} z_{11}^{u}, \quad z_{13}^{\varepsilon u 1}=\frac{z_{\alpha}^{(0)}}{R_{\alpha}}, \quad z_{13}^{\varepsilon u 2}=z_{\alpha}^{*(f)}, \quad z_{14}^{\varepsilon u}=z_{14}^{u} z_{\alpha}^{(0)}, \quad z_{22}^{\varepsilon u}=z_{22}^{(0)} z_{\beta}^{u}, \\
z_{23}^{\varepsilon u 1}=\frac{z_{\beta}^{(0)}}{R_{\beta}}, \quad z_{23}^{\varepsilon u 2}=z_{\beta}^{*(f)}, \quad z_{25}^{\varepsilon u}=z_{25}^{u} z_{\beta}^{(0)}, \quad z_{32}^{\varepsilon u}=\frac{\partial z_{22}^{u}}{\partial z}-\frac{z_{22}^{u} z_{\beta}^{(0)}}{R_{\beta}} \\
z_{33}^{\varepsilon u}=z_{\beta}^{(0)}+\frac{\partial z_{23}^{u}}{\partial z}-\frac{z_{23}^{u} z_{\beta}^{(0)}}{R_{\beta}}, \quad z_{35}^{\varepsilon u}=\frac{\partial z_{25}^{u}}{\partial z}-\frac{z_{25}^{u} z_{\beta}^{(0)}}{R_{\beta}}, \quad z_{41}^{\varepsilon u}=\frac{\partial z_{11}^{u}}{\partial z}-\frac{z_{11}^{u} z_{\alpha}^{(0)}}{R_{\alpha}} \\
z_{43}^{\varepsilon u}=z_{\alpha}^{(0)}+\frac{\partial z_{13}^{u}}{\partial z}-\frac{z_{13}^{u} z_{\alpha}^{(0)}}{R_{\alpha}}, \quad z_{44}^{\varepsilon u}=\frac{\partial z_{14}^{u}}{\partial z}-\frac{z_{14}^{u} z_{\alpha}^{(0)}}{R_{\alpha}}, \quad z_{51}^{\varepsilon u}=z_{11}^{u} z_{\beta}^{(0)} \\
z_{52}^{\varepsilon u}=z_{22}^{u} z_{\alpha}^{(0)}, \quad z_{53}^{\varepsilon u}=z_{13}^{u} z_{\beta}^{(0)}+z_{23}^{u} z_{\alpha}^{(0)}, \quad z_{54}^{\varepsilon u}=z_{14}^{u} z_{\beta}^{(0)}, \quad z_{55}^{\varepsilon u}=z_{25}^{u} z_{\alpha}^{(0)}
\end{gathered}
$$

and

$$
\begin{gathered}
z_{11}^{\varepsilon \tau}=z_{\alpha}^{(0)} z_{11}^{\tau}, \quad z_{12}^{\varepsilon \tau}=z_{\alpha}^{(0)} z_{12}^{\tau}, \quad z_{23}^{\varepsilon \tau}=z_{\beta}^{(0)} z_{23}^{\tau}, \quad z_{24}^{\varepsilon \tau}=z_{\beta}^{(0)} z_{24}^{\tau}, \quad z_{33}^{\varepsilon \tau}=\frac{\partial z_{23}^{\tau}}{\partial z}-\frac{z_{23}^{\tau}}{R_{\beta}} z_{\beta}^{(0)}, \\
z_{34}^{\varepsilon \tau}=\frac{\partial z_{24}^{\tau}}{\partial z}-\frac{z_{24}^{\tau}}{R_{\beta}} z_{\beta}^{(0)}, \quad z_{41}^{\varepsilon \tau}=\frac{\partial z_{11}^{\tau}}{\partial z}-\frac{z_{11}^{\tau}}{R_{\alpha}} z_{\alpha}^{(0)}, \quad z_{42}^{\varepsilon \tau}=\frac{\partial z_{12}^{\tau}}{\partial z}-\frac{z_{12}^{\tau}}{R_{\alpha}} z_{\alpha}^{(0)}, \\
z_{51}^{\varepsilon \tau}=z_{\beta}^{(0)} z_{11}^{\tau}, \quad z_{52}^{\varepsilon \tau}=z_{\beta}^{(0)} z_{12}^{\tau}, \quad z_{53}^{\varepsilon \tau}=z_{\alpha}^{(0)} z_{23}^{\tau}, \quad z_{54}^{\varepsilon \tau}=z_{\alpha}^{(0)} z_{24}^{\tau} .
\end{gathered}
$$

\section{E. Effective Strains Coefficients}

The modified (taking into account the electrical induced strains) terms of $\mathbf{z}^{\varepsilon u}(z)$ are

$$
\begin{array}{ll}
z_{13}^{\star \varepsilon u 1}=z_{13}^{u \varepsilon 1}-\bar{d}_{31}^{*} \bar{\lambda}\left[\bar{e}_{31}^{*}\left(z_{13}^{* u \varepsilon 1}-z_{13}^{u \varepsilon 1}\right)-\bar{e}_{32}^{*}\left(z_{23}^{* \varepsilon u 1}-z_{23}^{\varepsilon u 1}\right)\right], & z_{14}^{\star \varepsilon u}=z_{14}^{u \varepsilon}-\bar{d}_{31}^{*} \bar{\lambda} \bar{e}_{31}^{*}\left(z_{14}^{* u \varepsilon}-z_{14}^{u \varepsilon}\right), \\
z_{13}^{\star \varepsilon u 2}=z_{13}^{u \varepsilon 2}-\bar{d}_{31}^{*} \bar{\lambda} \bar{e}_{31}^{*}\left(z_{13}^{* u \varepsilon 2}-z_{13}^{u \varepsilon 2}\right)-\bar{d}_{31}^{*} \bar{\lambda} \bar{\lambda}_{32}^{*}\left(z_{23}^{* \varepsilon u 2}-z_{23}^{\varepsilon u 2}\right), & z_{15}^{* \varepsilon u}=-\bar{d}_{31}^{*} \bar{\lambda} \bar{e}_{32}^{*}\left(z_{25}^{* \varepsilon u}-z_{25}^{\varepsilon u}\right), \\
z_{23}^{\star \varepsilon u 1}=z_{23}^{\varepsilon u 1}-\bar{d}_{32}^{*} \bar{\lambda}\left[\bar{e}_{31}^{*}\left(z_{13}^{* u \varepsilon 1}-z_{13}^{u \varepsilon 1}\right)-\bar{e}_{32}^{*}\left(z_{23}^{* \varepsilon u 1}-z_{23}^{\varepsilon u 1}\right)\right], & z_{21}^{\star \varepsilon u}=-\bar{d}_{32}^{*} \bar{\lambda} \bar{e}_{31}^{*}\left(z_{11}^{* u \varepsilon}-z_{11}^{u \varepsilon}\right), \\
z_{23}^{\star \varepsilon u 2}=z_{23}^{u \varepsilon 2}-\bar{d}_{32}^{*} \bar{\lambda}\left[\bar{e}_{31}^{*}\left(z_{13}^{* u \varepsilon 1}-z_{13}^{u \varepsilon 1}\right)-\bar{e}_{32}^{*}\left(z_{23}^{* \varepsilon u 1}-z_{23}^{\varepsilon u 1}\right)\right], & z_{22}^{\star \varepsilon u}=z_{22}^{u \varepsilon}-\bar{d}_{32}^{*} \bar{\lambda} \bar{e}_{32}^{*}\left(z_{22}^{* \varepsilon u}-z_{22}^{\varepsilon u}\right), \\
z_{11}^{\star \varepsilon u}=z_{11}^{u \varepsilon}-\bar{d}_{31}^{*} \bar{\lambda} \bar{e}_{31}^{*}\left(z_{11}^{* u \varepsilon}-z_{11}^{u \varepsilon}\right), & z_{24}^{* \varepsilon u}=-\bar{d}_{32}^{*} \bar{\lambda} \bar{e}_{31}^{*}\left(z_{14}^{* u \varepsilon}-z_{14}^{u \varepsilon}\right), \\
z_{12}^{\star \varepsilon u}=-\bar{d}_{31}^{*} \bar{\lambda} \bar{e}_{32}^{*}\left(z_{22}^{* \varepsilon u}-z_{22}^{\varepsilon u}\right), & z_{25}^{* \varepsilon u}=z_{25}^{\varepsilon u}-\bar{d}_{32}^{*} \bar{\lambda} \bar{e}_{32}^{*}\left(z_{25}^{* \varepsilon u}-z_{25}^{\varepsilon u}\right),
\end{array}
$$

and of $\mathbf{z}^{\varepsilon \tau}(z)$ are

$$
\begin{array}{lll}
z_{11}^{\star \varepsilon \tau}=z_{11}^{\varepsilon \tau}-\bar{d}_{31}^{*} \bar{\lambda} \bar{e}_{31}^{*}\left(z_{11}^{* \varepsilon \tau}-z_{11}^{\varepsilon \tau}\right), & z_{12}^{\star \varepsilon \tau}=z_{12}^{\varepsilon \tau}-\bar{d}_{31}^{*} \bar{\lambda} \bar{e}_{31}^{*}\left(z_{12}^{* \varepsilon \tau}-z_{12}^{\varepsilon \tau}\right), \\
z_{13}^{\star \varepsilon \tau}=-\bar{d}_{31}^{*} \overline{\bar{e}} \bar{e}_{32}^{*}\left(z_{23}^{* \varepsilon \tau}-z_{23}^{\varepsilon \tau}\right), & z_{14}^{\star \varepsilon \tau}=-\bar{d}_{31}^{*} \bar{\lambda} \bar{e}_{32}^{*}\left(z_{24}^{* \varepsilon \tau}-z_{24}^{\varepsilon \tau}\right), \\
z_{21}^{\star \varepsilon \tau}=-\bar{d}_{32}^{*} \bar{\lambda} \bar{e}_{31}^{*}\left(z_{11}^{* \varepsilon \tau}-z_{11}^{\varepsilon \tau}\right), & & z_{23}^{\star \varepsilon \tau}=-\bar{d}_{32}^{*} \bar{\lambda} \bar{e}_{31}^{*}\left(z_{12}^{* \varepsilon \tau}-z_{12}^{\varepsilon \tau}\right), \\
z_{23}^{\star \varepsilon \tau}=z_{23}^{\varepsilon \tau}-\bar{d}_{32}^{*} \bar{\lambda} \bar{e}_{32}^{*}\left(z_{23}^{* \varepsilon \tau}-z_{23}^{\varepsilon \tau}\right), & & z_{24}^{\star \varepsilon \tau}=z_{24}^{\varepsilon \tau}-\bar{d}_{32}^{*} \bar{\lambda} \bar{e}_{32}^{*}\left(z_{24}^{* \varepsilon \tau}-z_{24}^{\varepsilon \tau}\right),
\end{array}
$$

where $\bar{\lambda}=\lambda / \bar{\epsilon}_{33}^{*}$ and the notation $z_{i j}^{*} \cdot$ denoting integration in order to $z$ as

$$
z_{i j}^{* \cdots}=\frac{1}{2 h} \int_{-h}^{+h} z_{i j} d z .
$$




\section{F. Virtual Work Terms}

\section{Virtual Work of the Inertial Forces}

Taking into account Eqs. (2) and the doubly-curved shells restrictions in Eqs. (39) into Eq. (52) yields

$$
\begin{aligned}
\delta T & =-\int_{\Omega_{0}}\left[\int_{-h}^{+h} \rho(\delta u \ddot{u}+\delta v \ddot{v}+\delta w \ddot{w}) \frac{1}{z_{\alpha}^{(0)} z_{\beta}^{(0)}} d z\right] d \alpha d \beta \\
& =-\int_{\Omega_{0}}\left[\delta u_{0}\left(I_{11}^{u u} \ddot{u}_{0}+I_{13}^{u u} \frac{\partial \ddot{w}_{0}}{\partial \alpha}+I_{14}^{u u} \ddot{\theta}_{0}^{\alpha}+I_{11}^{\tau u} \ddot{\bar{\tau}}_{z \alpha}+I_{21}^{\tau u} \ddot{\tilde{\tau}}_{z \alpha}\right)+\delta v_{0}\left(I_{22}^{u u} \ddot{v}_{0}+I_{23}^{u u} \frac{\partial \ddot{w}_{0}}{\partial \beta}\right.\right. \\
& \left.+I_{25}^{u u} \ddot{\theta}_{0}^{\beta}+I_{32}^{\tau u} \ddot{\bar{\tau}}_{\beta z}+I_{42}^{\tau u} \ddot{\tilde{\tau}}_{\beta z}\right)+\delta w\left(I_{33}^{u u} \ddot{w}\right)+\delta \frac{\partial w_{0}}{\partial \alpha}\left(I_{13}^{u u} \ddot{u}_{0}+I_{33}^{u \alpha} \frac{\partial \ddot{w}_{0}}{\partial \alpha}+I_{34}^{u u} \ddot{\theta}_{0}^{\alpha}+I_{13}^{\tau u} \ddot{\bar{\tau}}_{z \alpha}\right. \\
& \left.+I_{23}^{\tau} \ddot{\tilde{\tau}}_{z \alpha}\right)+\delta \frac{\partial w_{0}}{\partial \beta}\left(I_{23}^{u u} \ddot{v}_{0}+I_{33}^{u \beta} \frac{\partial \ddot{w}_{0}}{\partial \beta}+I_{35}^{u u} \ddot{\theta}_{0}^{\beta}+I_{33}^{\tau u} \ddot{\bar{\tau}}_{\beta z}+I_{43}^{\tau u} \ddot{\tilde{\tau}}_{\beta z}\right)+\delta \theta_{0}^{\alpha}\left(I_{14}^{u u} \ddot{u}_{0}\right. \\
& \left.+I_{43}^{u u} \frac{\partial \ddot{w}_{0}}{\partial \alpha}+I_{44}^{u u} \ddot{\theta}_{0}^{\alpha}+I_{14}^{\tau u} \ddot{\bar{\tau}}_{z \alpha}+I_{24}^{\tau u} \ddot{\tilde{\tau}}_{z \alpha}\right)+\delta \theta_{0}^{\beta}\left(I_{25}^{u u} \ddot{v}_{0}+I_{35}^{u u} \frac{\partial \ddot{w}_{0}}{\partial \beta}+I_{55}^{u u} \ddot{\theta}_{0}^{\beta}+I_{35}^{\tau u} \ddot{\bar{\tau}}_{\beta z}\right. \\
& \left.+I_{45}^{\tau u} \ddot{\tilde{\tau}}_{\beta z}\right)+\delta \bar{\tau}_{z \alpha}\left(I_{11}^{\tau u} \ddot{u}_{0}+I_{13}^{\tau u} \frac{\partial \ddot{w}_{0}}{\partial \alpha}+I_{14}^{\tau u} \ddot{\theta}_{0}^{\alpha}+I_{11}^{\tau \tau} \ddot{\bar{\tau}}_{z \alpha}+I_{12}^{\tau \tau} \ddot{\tilde{\tau}}_{z \alpha}\right)+\delta \tilde{\tau}_{z \alpha}\left(I_{21}^{\tau u} \ddot{u}_{0}\right. \\
& \left.+I_{23}^{\tau u} \frac{\partial \ddot{w}_{0}}{\partial \alpha}+I_{24}^{\tau u \ddot{\theta}_{0}^{\alpha}}+I_{12}^{\tau \tau} \ddot{\bar{\tau}}_{z \alpha}+I_{22}^{\tau \tau} \ddot{\tilde{\tau}}_{z \alpha}\right)+\delta \bar{\tau}_{\beta z}\left(I_{32}^{\tau u} \ddot{v}_{0}+I_{33}^{\tau u} \frac{\partial \ddot{w}_{0}}{\partial \beta}+I_{35}^{\tau u} \ddot{\theta}_{0}^{\beta}+I_{33}^{\tau \tau} \ddot{\bar{\tau}}_{\beta z}\right. \\
& \left.\left.+I_{34}^{\tau \tau} \ddot{\tilde{\tau}}_{\beta z}\right)+\delta \tilde{\tau}_{\beta z}\left(I_{42}^{\tau u} \ddot{v}_{0}+I_{43}^{\tau u} \frac{\partial \ddot{w}_{0}}{\partial \beta}+I_{45}^{\tau u} \ddot{\theta}_{0}^{\beta}+I_{34}^{\tau \tau} \ddot{\bar{\tau}}_{\beta z}+I_{44}^{\tau \tau} \ddot{\tilde{\tau}}_{z \alpha}\right)\right] d \alpha d \beta
\end{aligned}
$$

where

$$
\begin{aligned}
\left(I_{11}^{u u}, I_{13}^{u u}, I_{14}^{u u}\right) & =\rho\left\langle z_{11}^{u}\left(z_{11}^{u}, z_{13}^{u}, z_{14}^{u}\right)\right\rangle, \\
\left(I_{22}^{u u}, I_{23}^{u u}, I_{25}^{u u}\right) & =\rho\left\langle z_{22}^{u}\left(z_{22}^{u}, z_{23}^{u}, z_{25}^{u}\right)\right\rangle, \\
\left(I_{33}^{u u}\right) & =\rho\langle 1\rangle, \\
\left(I_{33}^{u \alpha}, I_{34}^{u u}\right) & =\rho\left\langle z_{13}^{u}\left(z_{13}^{u}, z_{14}^{u}\right)\right\rangle, \\
\left(I_{33}^{u \beta}, I_{35}^{u u}\right) & =\rho\left\langle z_{23}^{u}\left(z_{23}^{u}, z_{25}^{u}\right)\right\rangle, \\
\left(I_{44}^{u u}, I_{55}^{u u}\right) & =\rho\left\langle\left(z_{14}^{u} z_{14}^{u}, z_{25}^{u} z_{25}^{u}\right)\right\rangle, \\
\left(I_{11}^{\tau u}, I_{13}^{\tau u}, I_{14}^{\tau u}, I_{11}^{\tau \tau}, I_{12}^{\tau \tau}\right) & =\rho\left\langle z_{11}^{\tau}\left(z_{11}^{u}, z_{13}^{u}, z_{14}^{u}, z_{11}^{\tau}, z_{12}^{\tau}\right)\right\rangle, \\
\left(I_{21}^{\tau u}, I_{23}^{\tau u}, I_{24}^{\tau u}, I_{22}^{\tau \tau}\right) & =\rho\left\langle z_{12}^{\tau}\left(z_{11}^{u}, z_{13}^{u}, z_{14}^{u}, z_{12}^{\tau}\right)\right\rangle, \\
\left(I_{32}^{\tau u}, I_{33}^{\tau u}, I_{35}^{\tau u}, I_{33}^{\tau \tau}, I_{34}^{\tau \tau}\right) & =\rho\left\langle z_{23}^{\tau}\left(z_{22}^{u}, z_{23}^{u}, z_{25}^{u}, z_{23}^{\tau}, z_{34}^{\tau}\right)\right\rangle, \\
\left(I_{42}^{\tau u}, I_{43}^{\tau u}, I_{45}^{\tau u}, I_{44}^{\tau \tau}\right) & =\rho\left\langle z_{24}^{\tau}\left(z_{22}^{u}, z_{23}^{u}, z_{25}^{u}, z_{24}^{\tau}\right)\right\rangle,
\end{aligned}
$$

where for convenience $\langle\ldots\rangle$ denotes thickness integration and it is defined by

$$
\langle\ldots\rangle=\int_{-h}^{+h}(\ldots) \frac{1}{z_{\alpha}^{(0)} z_{\beta}^{(0)}} d z .
$$

\section{Virtual Work of the Internal Electro-Mechanical Forces}

Considering the strain definitions in Eq. (45), the term $\delta H_{\varepsilon}$ of Eq. (54) is given by

$$
\begin{aligned}
\delta H_{\varepsilon} & =\int_{\Omega_{0}}\left[\int_{-h}^{h}\left(\sigma_{\alpha \alpha} \delta \varepsilon_{\alpha \alpha}+\sigma_{\beta \beta} \delta \varepsilon_{\beta \beta}+\sigma_{\beta z} \delta \varepsilon_{\beta z}+\sigma_{z \alpha} \delta \varepsilon_{z \alpha}+\sigma_{\alpha \beta} \delta \varepsilon_{\alpha \beta}\right) \frac{1}{z_{\alpha}^{(0)} z_{\beta}^{(0)}} d z\right] d \alpha d \beta \\
& =\int_{\Omega_{0}}\left[\delta \frac{\partial u_{0}}{\partial \alpha}\left(N_{\alpha \alpha}^{\star 11}+N_{\beta \beta}^{\star 21}\right)+\delta \frac{\partial u_{0}}{\partial \beta}\left(N_{\alpha \beta}^{51}\right)+\delta u_{0}\left(Q_{z \alpha}^{41}\right)+\delta \frac{\partial v_{0}}{\partial \beta}\left(N_{\alpha \alpha}^{\star 12}+N_{\beta \beta}^{\star 22}\right)\right. \\
& +\delta \frac{\partial v_{0}}{\partial \alpha}\left(N_{\alpha \beta}^{52}\right)+\delta v_{0}\left(Q_{\beta z}^{32}\right)+\delta w_{0}\left(M_{\alpha \alpha}^{\star 131}+M_{\beta \beta}^{\star 231}\right)+\delta \frac{\partial^{2} w_{0}}{\partial \alpha^{2}}\left(M_{\alpha \alpha}^{\star 132}\right)+\delta \frac{\partial^{2} w_{0}}{\partial \beta^{2}}\left(M_{\beta \beta}^{\star 232}\right)
\end{aligned}
$$




$$
\begin{aligned}
& +\delta \frac{\partial^{2} w_{0}}{\partial \alpha \partial \beta}\left(M_{\alpha \beta}^{53}\right)+\delta \frac{\partial w_{0}}{\partial \alpha}\left(Q_{z \alpha}^{43}\right)+\delta \frac{\partial w_{0}}{\partial \beta}\left(Q_{\beta z}^{33}\right)+\delta \frac{\partial \theta_{\alpha}}{\partial \alpha}\left(M_{\alpha \alpha}^{\star 14}+M_{\beta \beta}^{\star 24}\right)+\delta \frac{\partial \theta_{\alpha}}{\partial \beta}\left(M_{\alpha \beta}^{54}\right) \\
& +\delta \theta_{\alpha}\left(Q_{z \alpha}^{44}\right)+\delta \frac{\partial \theta_{\beta}}{\partial \beta}\left(M_{\alpha \alpha}^{\star 15}+M_{\beta \beta}^{\star 25}\right)+\delta \frac{\partial \theta_{\beta}}{\partial \alpha}\left(M_{\alpha \beta}^{55}\right)+\delta \theta_{\beta}\left(Q_{\beta z}^{35}\right)+\delta \frac{\partial \bar{\tau}_{z \alpha}}{\partial \alpha}\left(T_{\alpha \alpha}^{\star 11}+T_{\beta \beta}^{\star 21}\right) \\
& +\delta \bar{\tau}_{z \alpha}\left(T_{z \alpha}^{41}\right)+\delta \frac{\partial \bar{\tau}_{z \alpha}}{\partial \beta}\left(T_{\alpha \beta}^{51}\right)+\delta \frac{\partial \tilde{\tau}_{z \alpha}}{\partial \alpha}\left(T_{\alpha \alpha}^{\star 12}+T_{\beta \beta}^{\star 22}\right)+\delta \tilde{\tau}_{z \alpha}\left(T_{z \alpha}^{42}\right)+\delta \frac{\partial \tilde{\tau}_{z \alpha}}{\partial \beta}\left(T_{\alpha \beta}^{52}\right) \\
& +\delta \frac{\partial \bar{\tau}_{\beta z}}{\partial \beta}\left(T_{\alpha \alpha}^{\star 13}+T_{\beta \beta}^{\star 23}\right)+\delta \bar{\tau}_{\beta z}\left(T_{\beta z}^{33}\right)+\delta \frac{\partial \bar{\tau}_{\beta z}}{\partial \alpha}\left(T_{\alpha \beta}^{53}\right)+\delta \frac{\partial \tilde{\tau}_{\beta z}}{\partial \beta}\left(T_{\alpha \alpha}^{\star 14}+T_{\beta \beta}^{\star 24}\right) \\
& \left.+\delta \tilde{\tau}_{\beta z}\left(T_{\beta z}^{34}\right)+\delta \frac{\partial \tilde{\tau}_{\beta z}}{\partial \alpha}\left(T_{\alpha \beta}^{54}\right)\right] d \alpha d \beta,
\end{aligned}
$$

where

$$
\begin{aligned}
\left(N_{\alpha \alpha}^{\star 11}, N_{\alpha \alpha}^{\star 12}, M_{\alpha \alpha}^{\star 131}, M_{\alpha \alpha}^{\star 132}, M_{\alpha \alpha}^{\star 14}, M_{\alpha \alpha}^{\star 15}\right) & =\left\langle\sigma_{\alpha \alpha}\left(z_{11}^{\star \varepsilon u}, z_{12}^{\star \varepsilon u}, z_{13}^{\star \varepsilon u 1}, z_{13}^{\star \varepsilon u 2}, z_{14}^{\star \varepsilon u}, z_{15}^{\star \varepsilon u}\right)\right\rangle, \\
\left(N_{\beta \beta}^{\star 21}, N_{\beta \beta}^{\star 22}, M_{\beta \beta}^{\star 231}, M_{\beta \beta}^{\star 232}, M_{\beta \beta}^{\star 24}, M_{\beta \beta}^{\star 25}\right) & =\left\langle\sigma_{\beta \beta}\left(z_{21}^{\star \varepsilon u}, z_{22}^{\star \varepsilon u}, z_{23}^{\star \varepsilon u 1}, z_{23}^{\star \varepsilon u 2}, z_{24}^{\star \varepsilon u}, z_{25}^{\star \varepsilon u}\right)\right\rangle, \\
\left(Q_{\beta z}^{32}, Q_{\beta z}^{33}, Q_{\beta z}^{35}\right) & =\left\langle\sigma_{\beta z}\left(z_{32}^{\varepsilon u}, z_{33}^{\varepsilon u}, z_{35}^{\varepsilon u}\right)\right\rangle, \\
\left(Q_{z \alpha}^{41}, Q_{z \alpha}^{43}, Q_{z \alpha}^{44}\right) & =\left\langle\sigma_{z \alpha}\left(z_{41}^{\varepsilon u}, z_{43}^{\varepsilon u}, z_{44}^{\varepsilon u}\right)\right\rangle, \\
\left(N_{\alpha \beta}^{51}, N_{\alpha \beta}^{52}, M_{\alpha \beta}^{53}, M_{\alpha \beta}^{54}, M_{\alpha \beta}^{55}\right) & =\left\langle\sigma_{\alpha \beta}\left(z_{51}^{\varepsilon u}, z_{52}^{\varepsilon u}, z_{53}^{\varepsilon u}, z_{54}^{\varepsilon u}, z_{55}^{\varepsilon u}\right)\right\rangle, \\
\left(T_{\alpha \alpha}^{\star 11}, T_{\alpha \alpha}^{\star 12}, T_{\alpha \alpha}^{\star 13}, T_{\alpha \alpha}^{\star 14}\right) & =\left\langle\sigma_{\alpha \alpha}\left(z_{11}^{\star \varepsilon \tau}, z_{12}^{\star \varepsilon \tau}, z_{13}^{\star \varepsilon \tau}, z_{14}^{\star \varepsilon \tau}\right)\right\rangle, \\
\left(T_{\beta \beta}^{\star 21}, T_{\beta \beta}^{\star 22}, T_{\beta \beta}^{\star 23}, T_{\beta \beta}^{\star 24}\right) & =\left\langle\sigma_{\beta \beta}\left(z_{21}^{\star \varepsilon \tau}, z_{22}^{\star \varepsilon \tau}, z_{23}^{\star \varepsilon \tau}, z_{24}^{\star \varepsilon \tau}\right)\right\rangle, \\
\left(T_{\beta z}^{33}, T_{\beta z}^{34}\right) & =\left\langle\sigma_{\beta z}\left(z_{33}^{\varepsilon \tau}, z_{34}^{\varepsilon \tau}\right)\right\rangle, \\
\left(T_{z \alpha}^{41}, T_{z \alpha}^{42}\right) & =\left\langle\sigma_{z \alpha}\left(z_{41}^{\varepsilon \tau}, z_{42}^{\varepsilon \tau}\right)\right\rangle, \\
\left(T_{\alpha \beta}^{51}, T_{\alpha \beta}^{52}, T_{\alpha \beta}^{53}, T_{\alpha \beta}^{54}\right) & =\left\langle\sigma_{\alpha \beta}\left(z_{51}^{\varepsilon \tau}, z_{52}^{\varepsilon \tau}, z_{53}^{\varepsilon \tau}, z_{54}^{\varepsilon \tau}\right)\right\rangle .
\end{aligned}
$$

Since the direct piezoelectric effect (the stiffness increase due to the induced electric field) was already considered in Sec. C, the electric enthalpy term $\delta H_{E}$ will only consider the applied electric potential counterpart of the transverse electric displacement $D_{z}$ and electric field $E_{z}$ defined in Eqs. (36) and (37). Thus, considering (C16) and an orthotropic layer, the second term of Eq. (54), $\delta H_{E}$, is given by

$$
\begin{aligned}
\delta H_{E} & =\int_{\Omega_{0}}\left[\int_{-h}^{+h} \delta \phi\left(-\frac{\lambda \bar{e}_{31}^{*}}{2 h} \varepsilon_{\alpha \alpha}-\frac{\lambda \bar{e}_{32}^{*}}{2 h} \varepsilon_{\beta \beta}+\frac{\lambda^{2} \bar{\epsilon}_{33}^{*}}{4 h^{2}} \phi\right) \frac{1}{z_{\alpha}^{(0)} z_{\beta}^{(0)}} d z\right] d \alpha d \beta \\
& =\int_{\Omega_{0}} \delta \phi\left[\left(P_{\alpha \alpha}^{\star 11}+P_{\beta \beta}^{\star 21}\right) \frac{\partial u_{0}}{\partial \alpha}+\left(P_{\alpha \alpha}^{\star 12}+P_{\beta \beta}^{\star 22}\right) \frac{\partial v_{0}}{\partial \beta}+\left(P_{\alpha \alpha 1}^{\star 13}+P_{\alpha \alpha 1}^{\star 23}\right) w_{0}+\left(P_{\alpha \alpha 2}^{\star 13}\right) \frac{\partial^{2} w_{0}}{\partial \alpha^{2}}\right. \\
& +\left(P_{\beta \beta 2}^{\star 23}\right) \frac{\partial^{2} w_{0}}{\partial \beta^{2}}+\left(P_{\alpha \alpha}^{\star 14}+P_{\beta \beta}^{\star 24}\right) \frac{\partial \theta_{\alpha}}{\partial \alpha}+\left(P_{\alpha \alpha}^{\star 15}+P_{\beta \beta}^{\star 25}\right) \frac{\partial \theta_{\beta}}{\partial \beta}+\left(R_{\alpha \alpha}^{\star 11}+R_{\beta \beta}^{\star 21}\right) \frac{\partial \bar{\tau}_{z \alpha}}{\partial \alpha} \\
& \left.+\left(R_{\alpha \alpha}^{\star 12}+R_{\beta \beta}^{\star 22}\right) \frac{\partial \tilde{\tau}_{z \alpha}}{\partial \alpha}+\left(R_{\alpha \alpha}^{\star 13}+R_{\beta \beta}^{\star 23}\right) \frac{\partial \bar{\tau}_{\beta z}}{\partial \beta}+\left(R_{\alpha \alpha}^{\star 14}+R_{\beta \beta}^{\star 24}\right) \frac{\partial \tilde{\tau}_{\beta z}}{\partial \beta}+\left(S_{\phi \phi}\right) \phi\right] d \alpha d \beta
\end{aligned}
$$

where

$$
\begin{aligned}
\left(P_{\alpha \alpha}^{\star 11}, P_{\alpha \alpha}^{\star 12}, P_{\alpha \alpha 1}^{\star 13}, P_{\alpha \alpha 2}^{\star 13}, P_{\alpha \alpha}^{\star 14}, P_{\alpha \alpha}^{\star 15}\right) & =\left\langle\frac{\lambda \bar{e}_{31}^{*}}{2 h}\left(z_{11}^{\star \varepsilon u}, z_{12}^{\star \varepsilon u}, z_{13}^{\star \varepsilon u 1}, z_{13}^{\star \varepsilon u 2}, z_{14}^{\star \varepsilon u}, z_{15}^{\star \varepsilon u}\right)\right\rangle, \\
\left(P_{\beta \beta}^{\star 21}, P_{\beta \beta}^{\star 22}, P_{\beta \beta 1}^{\star 23}, P_{\beta \beta 2}^{\star 23}, P_{\beta \beta}^{\star 24}, P_{\beta \beta}^{\star 25}\right) & =\left\langle\frac{\lambda \bar{e}_{32}^{*}}{2 h}\left(z_{21}^{\star \varepsilon u}, z_{22}^{\star \varepsilon u}, z_{23}^{\star \varepsilon u 1}, z_{23}^{\star \varepsilon u 2}, z_{24}^{\star \varepsilon u}, z_{25}^{\star \varepsilon u}\right)\right\rangle, \\
\left(R_{\alpha \alpha}^{\star 11}, R_{\alpha \alpha}^{\star 12}, R_{\alpha \alpha}^{\star 13}, R_{\alpha \alpha}^{\star 14}\right) & =\left\langle\frac{\lambda \bar{e}_{31}^{*}}{2 h}\left(z_{11}^{\star \varepsilon \tau}, z_{12}^{\star \varepsilon \tau}, z_{13}^{\star \varepsilon \tau}, z_{14}^{\star \varepsilon \tau}\right)\right\rangle, \\
\left(R_{\beta \beta}^{\star 21}, R_{\beta \beta}^{\star 22}, R_{\beta \beta}^{\star 23}, R_{\beta \beta}^{\star 24}\right) & =\left\langle\frac{\lambda \bar{e}_{32}^{*}}{2 h}\left(z_{21}^{\star \varepsilon \tau}, z_{22}^{\star \varepsilon \tau}, z_{23}^{\star \varepsilon \tau}, z_{24}^{\star \varepsilon \tau}\right)\right\rangle, \\
S_{\phi \phi} & =\left\langle\frac{\lambda^{2} \bar{\epsilon}_{33}^{*}}{4 h^{2}}\right\rangle .
\end{aligned}
$$




\section{Virtual Work of the Non-Conservative Forces}

In a similar way to what has been done before, the integration with respect to $z$ is conveniently carrying out, and the virtual work of the non-conservative forces $\delta W_{u}$ can be expressed in terms of prescribed forces and moments as

$$
\begin{aligned}
\delta W_{u} & =\int_{\Omega_{0}} \delta w_{0}(Z) d \alpha d \beta+\oint_{\Gamma_{\alpha}}\left[\delta u_{0}\left(\hat{N}_{\beta \alpha}^{11}\right)+\delta v_{0}\left(\hat{N}_{\beta \beta}^{22}\right)+\delta \frac{\partial w_{0}}{\partial \beta}\left(\hat{M}_{\beta \beta}^{23}\right)+\delta \frac{\partial w_{0}}{\partial \alpha}\left(\hat{M}_{\beta \alpha}^{13}\right)+\delta w_{0}\left(\hat{Q}_{\beta z}^{33}\right)\right. \\
& \left.+\delta \theta_{\alpha}\left(\hat{M}_{\beta \alpha}^{14}\right)+\delta \theta_{\beta}\left(\hat{M}_{\beta \beta}^{25}\right)+\delta \bar{\tau}_{z \alpha}\left(\hat{T}_{\beta \alpha}^{11}\right)+\delta \tilde{\tau}_{z \alpha}\left(\hat{T}_{\beta \alpha}^{12}\right)+\delta \bar{\tau}_{\beta z}\left(\hat{T}_{\beta \beta}^{23}\right)+\delta \tilde{\tau}_{\beta z}\left(\hat{T}_{\beta \beta}^{24}\right)\right] d \alpha \\
& +\oint_{\Gamma_{\beta}}\left[\delta u_{0}\left(\hat{N}_{\alpha \alpha}^{11}\right)+\delta v_{0}\left(\hat{N}_{\alpha \beta}^{22}\right)+\delta \frac{\partial w_{0}}{\partial \alpha}\left(\hat{M}_{\alpha \alpha}^{13}\right)+\delta \frac{\partial w_{0}}{\partial \beta}\left(\hat{M}_{\alpha \alpha}^{23}\right)+\delta w_{0}\left(\hat{Q}_{z \alpha}^{33}\right)+\delta \theta_{\alpha}\left(\hat{M}_{\alpha \alpha}^{14}\right)\right. \\
& \left.+\delta \theta_{\beta}\left(\hat{M}_{\alpha \beta}^{25}\right)+\delta \bar{\tau}_{z \alpha}\left(\hat{T}_{\alpha \alpha}^{11}\right)+\delta \tilde{\tau}_{z \alpha}\left(\hat{T}_{\alpha \alpha}^{12}\right)+\delta \bar{\tau}_{\beta z}\left(\hat{T}_{\alpha \beta}^{23}\right)+\delta \tilde{\tau}_{\beta z}\left(\hat{T}_{\alpha \beta}^{24}\right)\right] d \beta,
\end{aligned}
$$

where

$$
\begin{aligned}
\left(\hat{N}_{\alpha \alpha}^{11}, \hat{M}_{\alpha \alpha}^{13}, \hat{M}_{\alpha \alpha}^{14}\right) & =\left\langle\hat{\sigma}_{\alpha \alpha}\left(z_{11}^{u}, z_{13}^{u}, z_{14}^{u}\right)\right\rangle_{\beta}, \\
\left(\hat{N}_{\alpha \beta}^{22}, \hat{M}_{\alpha \beta}^{23}, \hat{M}_{\alpha \beta}^{25}\right) & =\left\langle\hat{\sigma}_{\alpha \beta}\left(z_{22}^{u}, z_{23}^{u}, z_{25}^{u}\right)\right\rangle_{\beta}, \\
\left(\hat{Q}_{z \alpha}^{33}\right) & =\left\langle\hat{\sigma}_{z \alpha}\right\rangle_{\beta}, \\
\left(\hat{T}_{\alpha \alpha}^{11}, \hat{T}_{\alpha \alpha}^{12}\right) & =\left\langle\hat{\sigma}_{\alpha \alpha}\left(z_{11}^{\tau}, z_{12}^{\tau}\right)\right\rangle_{\beta}, \\
\left(\hat{T}_{\alpha \beta}^{23}, \hat{T}_{\alpha \beta}^{24}\right) & =\left\langle\hat{\sigma}_{\alpha \beta}\left(z_{23}^{\tau}, z_{24}^{\tau}\right)\right\rangle_{\beta}, \\
\left(\hat{N}_{\beta \beta}^{22}, \hat{M}_{\beta \beta}^{23}, \hat{M}_{\beta \beta}^{25}\right) & =\left\langle\hat{\sigma}_{\beta \beta}\left(z_{22}^{u}, z_{23}^{u}, z_{25}^{u}\right)\right\rangle_{\alpha}, \\
\left(\hat{N}_{\beta \alpha}^{11}, \hat{M}_{\beta \alpha}^{13}, \hat{M}_{\beta \alpha}^{14}\right) & =\left\langle\hat{\sigma}_{\beta \alpha}\left(z_{11}^{u}, z_{13}^{u}, z_{14}^{u}\right)\right\rangle_{\alpha}, \\
\left(\hat{Q}_{\beta z}^{33}\right) & =\left\langle\hat{\sigma}_{\beta z}\right\rangle_{\alpha}, \\
\left(\hat{T}_{\beta \beta}^{23}, \hat{T}_{\beta \beta}^{24}\right) & =\left\langle\hat{\sigma}_{\beta \beta}\left(z_{23}^{\tau}, z_{24}^{\tau}\right)\right\rangle_{\alpha}, \\
\left(\hat{T}_{\beta \alpha}^{11}, \hat{T}_{\beta \alpha}^{12}\right) & =\left\langle\hat{\sigma}_{\beta \alpha}\left(z_{11}^{\tau}, z_{12}^{\tau}\right)\right\rangle_{\alpha} .
\end{aligned}
$$

Once again, for convenience, $\langle\ldots\rangle_{\alpha}$ and $\langle\ldots\rangle_{\beta}$ denote thickness integration and are defined as

$$
\langle\ldots\rangle_{\alpha}=\int_{-h}^{+h}(\ldots) \frac{1}{z_{\alpha}^{(0)}} d z,\langle\ldots\rangle_{\beta}=\int_{-h}^{+h}(\ldots) \frac{1}{z_{\beta}^{(0)}} d z .
$$

\section{G. Internal Forces and Moments in Terms of Generalized Variables}

$$
\begin{aligned}
& \left\{\begin{array}{c}
\left(N_{\alpha \alpha}^{\star 11}, N_{\alpha \alpha}^{\star 12}\right) \\
\left(N_{\beta \beta}^{\star 21}, N_{\beta \beta}^{\star 22}\right) \\
\left(N_{\alpha \beta}^{51}, N_{\alpha \beta}^{52}\right)
\end{array}\right\}=\left[\begin{array}{ccccc}
A_{11} \partial_{\alpha} & A_{12} \partial_{\beta} & A_{13}^{1}+A_{13}^{2} \partial_{\alpha \alpha}+A_{13}^{3} \partial_{\beta \beta} & A_{14} \partial_{\alpha} & A_{15} \partial_{\beta} \\
A_{21} \partial_{\alpha} & A_{22} \partial_{\beta} & A_{23}^{1}+A_{23}^{2} \partial_{\alpha \alpha}+A_{23}^{3} \partial_{\beta \beta} & A_{24} \partial_{\alpha} & A_{25} \partial_{\beta} \\
A_{51} \partial_{\beta} & A_{52} \partial_{\alpha} & A_{53} \partial_{\alpha \beta} & A_{54} \partial_{\beta} & A_{55} \partial_{\alpha}
\end{array}\right]\left\{\begin{array}{c}
u_{0} \\
v_{0} \\
w_{0} \\
\theta_{\alpha} \\
\theta_{\beta}
\end{array}\right\} \\
& +\left[\begin{array}{cccc}
A_{16} \partial_{\alpha} & A_{17} \partial_{\alpha} & A_{18} \partial_{\beta} & A_{19} \partial_{\beta} \\
A_{26} \partial_{\alpha} & A_{27} \partial_{\alpha} & A_{28} \partial_{\beta} & A_{29} \partial_{\beta} \\
A_{56} \partial_{\beta} & A_{57} \partial_{\beta} & A_{58} \partial_{\alpha} & A_{59} \partial_{\alpha}
\end{array}\right]\left\{\begin{array}{c}
\bar{\tau}_{z \alpha} \\
\tilde{\tau}_{z \alpha} \\
\bar{\tau}_{\beta z} \\
\tilde{\tau}_{\beta z}
\end{array}\right\}+\left\{\begin{array}{c}
\left(P_{\alpha \alpha}^{\star 11}, P_{\alpha \alpha}^{\star 12}\right) \\
\left(P_{\beta \beta}^{\star 21}, P_{\beta \beta}^{\star 22}\right) \\
0
\end{array}\right\} \phi \\
& \left\{\left(\begin{array}{c}
\left(Q_{\beta z}^{32}, Q_{\beta z}^{33}, Q_{\beta z}^{35}\right) \\
\left(Q_{z \alpha}^{41}, Q_{z \alpha}^{43}, Q_{z \alpha}^{44}\right)
\end{array}\right\}=\left[\begin{array}{ccccc}
0 & A_{32} & A_{33} \partial_{\beta} & 0 & A_{35} \\
A_{41} & 0 & A_{43} \partial_{\alpha} & A_{44} & 0
\end{array}\right]\left\{\begin{array}{c}
u_{0} \\
v_{0} \\
w_{0} \\
\theta_{\alpha} \\
\theta_{\beta}
\end{array}\right\}+\left[\begin{array}{cccc}
0 & 0 & A_{38} & A_{39} \\
A_{46} & A_{47} & 0 & 0
\end{array}\right]\left\{\begin{array}{c}
\bar{\tau}_{z \alpha} \\
\tilde{\tau}_{z \alpha} \\
\bar{\tau}_{\beta z} \\
\tilde{\tau}_{\beta z}
\end{array}\right\},\right.
\end{aligned}
$$




$$
\begin{aligned}
& \left\{\begin{array}{c}
\left(M_{\alpha \alpha}^{\star 131}, M_{\alpha \alpha}^{\star 132}, M_{\alpha \alpha}^{\star 14}, M_{\alpha \alpha}^{\star 15}\right) \\
\left(M_{\beta \beta}^{\star 231}, M_{\beta \beta}^{\star 232}, M_{\beta \beta}^{\star 24}, M_{\beta \beta}^{\star 25}\right) \\
\left(M_{\alpha \beta}^{53}, M_{\alpha \beta}^{54}, M_{\alpha \beta}^{55}\right)
\end{array}\right\}=\left[\begin{array}{ccccc}
B_{11} \partial_{\alpha} & B_{12} \partial_{\beta} & B_{13}^{1}+B_{13}^{2} \partial_{\alpha \alpha}+B_{13}^{3} \partial_{\beta \beta} & B_{14} \partial_{\alpha} & B_{15} \partial_{\beta} \\
B_{21} \partial_{\alpha} & B_{22} \partial_{\beta} & B_{23}^{1}+B_{23}^{2} \partial_{\alpha \alpha}+B_{23}^{3} \partial_{\beta \beta} & B_{24} \partial_{\alpha} & B_{25} \partial_{\beta} \\
B_{51} \partial_{\beta} & B_{52} \partial_{\alpha} & B_{53} \partial_{\alpha \beta} & B_{54} \partial_{\beta} & B_{55} \partial_{\alpha}
\end{array}\right]\left\{\begin{array}{c}
u_{0} \\
v_{0} \\
w_{0} \\
\theta_{\alpha} \\
\theta_{\beta}
\end{array}\right\} \\
& +\left[\begin{array}{cccc}
B_{16} \partial_{\alpha} & B_{17} \partial_{\alpha} & B_{18} \partial_{\beta} & B_{19} \partial_{\beta} \\
B_{26} \partial_{\alpha} & B_{27} \partial_{\alpha} & B_{28} \partial_{\beta} & B_{29} \partial_{\beta} \\
B_{56} \partial_{\beta} & B_{57} \partial_{\beta} & B_{58} \partial_{\alpha} & B_{59} \partial_{\alpha}
\end{array}\right]\left\{\begin{array}{c}
\bar{\tau}_{z \alpha} \\
\tilde{\tau}_{z \alpha} \\
\bar{\tau}_{\beta z} \\
\tilde{\tau}_{\beta z}
\end{array}\right\}+\left\{\begin{array}{c}
\left(P_{\alpha \alpha}^{\star 131}, P_{\alpha \alpha}^{\star 132}, P_{\alpha \alpha}^{\star 14}, P_{\alpha \alpha}^{\star 15}\right) \\
\left(P_{\beta \beta}^{\star 231}, P_{\beta \beta}^{\star 232}, P_{\beta \beta}^{\star 24}, P_{\beta \beta}^{\star 25}\right) \\
0
\end{array}\right\} \phi \\
& \left\{\begin{array}{c}
\left(T_{\alpha \alpha}^{\star 11}, T_{\alpha \alpha}^{\star 12}, T_{\alpha \alpha}^{\star 13}, T_{\alpha \alpha}^{\star 14}\right) \\
\left(T_{\beta \beta}^{\star 21}, T_{\beta \beta}^{\star 22}, T_{\beta \beta}^{\star 23}, T_{\beta \beta}^{\star 24}\right) \\
\left(T_{\alpha \beta}^{51}, T_{\alpha \beta}^{52}, T_{\alpha \beta}^{53}, T_{\alpha \beta}^{54}\right)
\end{array}\right\}=\left[\begin{array}{ccccc}
C_{11} \partial_{\alpha} & C_{12} \partial_{\beta} & C_{13}^{1}+C_{13}^{2} \partial_{\alpha \alpha}+C_{13}^{3} \partial_{\beta \beta} & C_{14} \partial_{\alpha} & C_{15} \partial_{\beta} \\
C_{21} \partial_{\alpha} & C_{22} \partial_{\beta} & C_{23}^{1}+C_{23}^{2} \partial_{\alpha \alpha}+C_{23}^{3} \partial_{\beta \beta} & C_{24} \partial_{\alpha} & C_{25} \partial_{\beta} \\
C_{51} \partial_{\beta} & C_{52} \partial_{\alpha} & C_{53} \partial_{\alpha \beta} & C_{54} \partial_{\beta} & C_{55} \partial_{\alpha}
\end{array}\right]\left\{\begin{array}{c}
u_{0} \\
v_{0} \\
w_{0} \\
\theta_{\alpha} \\
\theta_{\beta}
\end{array}\right\} \\
& +\left[\begin{array}{cccc}
C_{16} \partial_{\alpha} & C_{17} \partial_{\alpha} & C_{18} \partial_{\beta} & C_{19} \partial_{\beta} \\
C_{26} \partial_{\alpha} & C_{27} \partial_{\alpha} & C_{28} \partial_{\beta} & C_{29} \partial_{\beta} \\
C_{56} \partial_{\beta} & C_{57} \partial_{\beta} & C_{58} \partial_{\alpha} & C_{59} \partial_{\alpha}
\end{array}\right]\left\{\begin{array}{c}
\bar{\tau}_{z \alpha} \\
\tilde{\tau}_{z \alpha} \\
\bar{\tau}_{\beta z} \\
\tilde{\tau}_{\beta z}
\end{array}\right\}+\left\{\begin{array}{c}
\left(R_{\alpha \alpha}^{\star 11}, R_{\alpha \alpha}^{\star 12}, R_{\alpha \alpha}^{\star 13}, R_{\alpha \alpha}^{\star 14}\right) \\
\left(R_{\beta \beta}^{\star 21}, R_{\beta \beta}^{\star 22}, R_{\beta \beta}^{\star 23}, R_{\beta \beta}^{\star 24}\right) \\
0
\end{array}\right\} \phi, \\
& \left\{\begin{array}{l}
\left(T_{\beta z}^{33}, T_{\beta z}^{34}\right) \\
\left(T_{z \alpha}^{41}, T_{z \alpha}^{42}\right)
\end{array}\right\}=\left[\begin{array}{ccccc}
0 & C_{32} & C_{33} \partial_{\beta} & 0 & C_{35} \\
C_{41} & 0 & C_{43} \partial_{\alpha} & C_{44} & 0
\end{array}\right]\left\{\begin{array}{c}
u_{0} \\
v_{0} \\
w_{0} \\
\theta_{\alpha} \\
\theta_{\beta}
\end{array}\right\}+\left[\begin{array}{cccc}
0 & 0 & C_{38} & C_{39} \\
C_{46} & C_{47} & 0 & 0
\end{array}\right]\left\{\begin{array}{l}
\bar{\tau}_{z \alpha} \\
\tilde{\tau}_{z \alpha} \\
\bar{\tau}_{\beta z} \\
\tilde{\tau}_{\beta z}
\end{array}\right\}
\end{aligned}
$$

Considering the generic internal force or moment terms in Eqs. (73),(77) and (78) as

$$
\left\langle\left[\begin{array}{ccc}
\bar{c}_{11}^{*} & \bar{c}_{12}^{*} & 0 \\
\bar{c}_{12}^{*} & \bar{c}_{22}^{*} & 0 \\
0 & 0 & \bar{c}_{66}^{*}
\end{array}\right]\left\{\begin{array}{l}
\varepsilon_{\alpha \alpha}\left(g_{1}\right) \\
\varepsilon_{\beta \beta}\left(g_{2}\right) \\
\varepsilon_{\alpha \beta}\left(g_{5}\right)
\end{array}\right\}\right\rangle,
$$

where $g_{i}$ is used to denote a series of coefficients $\left(g_{i 1}, g_{i 2}, \ldots\right)$ of the correspondent $z$ 's used to define the internal forces and moments, the following rule holds to determine the $A_{i j}, B_{i j}$ and $C_{i j}$ coefficients (with $i=1,2,5$ and $j=1, \ldots, 9)$, denoted by the generic $G_{i j}$,

$$
\begin{aligned}
& G_{11}=\left\langle\bar{c}_{11}^{*} z_{11}^{\star \varepsilon u} g_{1}+\bar{c}_{12}^{*} z_{21}^{u \star \varepsilon} g_{2}\right\rangle, \quad G_{23}^{3}=\left\langle\bar{c}_{22}^{*} z_{23}^{\star \varepsilon u 2} g_{1}\right\rangle, \\
& G_{12}=\left\langle\bar{c}_{11}^{*} z_{12}^{\star \varepsilon u} g_{1}+\bar{c}_{12}^{*} z_{22}^{\star \varepsilon u} g_{2}\right\rangle, \quad G_{24}=\left\langle\bar{c}_{12}^{*} z_{14}^{\star \varepsilon u} g_{1}+\bar{c}_{22}^{*} z_{24}^{\star \varepsilon u} g_{2}\right\rangle, \\
& G_{13}^{1}=\left\langle\bar{c}_{11}^{*} z_{13}^{\star \varepsilon u 1} g_{1}+\bar{c}_{12}^{*} z_{23}^{\star \varepsilon u 1} g_{2}\right\rangle, \quad G_{25}=\left\langle\bar{c}_{12}^{*} z_{15}^{\star \varepsilon u} g_{1}+\bar{c}_{22}^{*} z_{25}^{\star \varepsilon u} g_{2}\right\rangle, \\
& G_{13}^{2}=\left\langle\bar{c}_{11}^{*} z_{13}^{\star \varepsilon u 2} g_{1}\right\rangle \text {, } \\
& G_{51}=\left\langle\bar{c}_{66}^{*} z_{51}^{\varepsilon u} g_{5}\right\rangle \text {, } \\
& G_{13}^{3}=\left\langle\bar{c}_{12}^{*} z_{23}^{\star \varepsilon u 2} g_{1}\right\rangle \text {, } \\
& G_{52}=\left\langle\bar{c}_{66}^{*} z_{52}^{\varepsilon u} g_{5}\right\rangle, \\
& G_{14}=\left\langle\bar{c}_{11}^{*} z_{14}^{\star \varepsilon u} g_{1}+\bar{c}_{12}^{*} z_{24}^{\star \varepsilon u} g_{2}\right\rangle, \\
& G_{53}=\left\langle\bar{c}_{66}^{*} z_{53}^{\varepsilon u} g_{5}\right\rangle, \\
& G_{15}=\left\langle\bar{c}_{11}^{*} z_{15}^{\star \varepsilon u} g_{1}+\bar{c}_{12}^{*} z_{25}^{\star \varepsilon u} g_{2}\right\rangle \text {, } \\
& G_{54}=\left\langle\bar{c}_{66}^{*} z_{54}^{\varepsilon u} g_{5}\right\rangle, \\
& G_{18}=\left\langle\bar{c}_{11}^{*} z_{13}^{\star \varepsilon \tau} g_{1}+\bar{c}_{12}^{*} z_{23}^{\star \varepsilon \tau} g_{2}\right\rangle, \\
& G_{19}=\left\langle\bar{c}_{11}^{*} z_{14}^{\star \varepsilon \tau} g_{1}+\bar{c}_{12}^{*} z_{24}^{\star \varepsilon \tau} g_{2}\right\rangle, \\
& G_{26}=\left\langle\bar{c}_{12}^{*} z_{11}^{\star \varepsilon \tau} g_{1}+\bar{c}_{22}^{*} z_{21}^{\star \varepsilon \tau} g_{2}\right\rangle, \\
& G_{27}=\left\langle\bar{c}_{12}^{*} z_{12}^{\star \varepsilon \tau} g_{1}+\bar{c}_{22}^{*} z_{22}^{\star \varepsilon \tau} g_{2}\right\rangle, \\
& G_{28}=\left\langle\bar{c}_{12}^{*} z_{13}^{\star \varepsilon \tau} g_{1}+\bar{c}_{22}^{*} z_{23}^{\star \varepsilon \tau} g_{2}\right\rangle \text {, } \\
& G_{29}=\left\langle\bar{c}_{12}^{*} z_{14}^{\star \varepsilon \tau} g_{1}+\bar{c}_{22}^{*} z_{24}^{\star \varepsilon \tau} g_{2}\right\rangle, \\
& G_{56}=\left\langle\bar{c}_{66}^{*} z_{51}^{\varepsilon \tau} g_{5}\right\rangle \text {, } \\
& G_{21}=\left\langle\bar{c}_{12}^{*} z_{11}^{\star \varepsilon u} g_{1}+\bar{c}_{22}^{*} z_{21}^{\star \varepsilon u} g_{2}\right\rangle, \\
& G_{55}=\left\langle\bar{c}_{66}^{*} z_{55}^{\varepsilon u} g_{5}\right\rangle \text {, } \\
& G_{57}=\left\langle\bar{c}_{66}^{*} z_{52}^{\varepsilon \tau} g_{5}\right\rangle, \\
& G_{22}=\left\langle\bar{c}_{12}^{*} z_{12}^{\star \varepsilon u} g_{1}+\bar{c}_{22}^{*} z_{22}^{\star \varepsilon u} g_{2}\right\rangle, \\
& G_{16}=\left\langle\bar{c}_{11}^{*} z_{11}^{\star \varepsilon \tau} g_{1}+\bar{c}_{12}^{*} z_{21}^{\star \varepsilon \tau} g_{2}\right\rangle \text {, } \\
& G_{58}=\left\langle\bar{c}_{66}^{*} z_{53}^{\varepsilon \tau} g_{5}\right\rangle \text {, } \\
& G_{23}^{1}=\left\langle\bar{c}_{12}^{*} z_{13}^{\star \varepsilon u 1} g_{1}+\bar{c}_{22}^{*} z_{23}^{\star \varepsilon u 1} g_{2}\right\rangle, \quad G_{17}=\left\langle\bar{c}_{11}^{*} z_{12}^{\star \varepsilon \tau} g_{1}+\bar{c}_{12}^{*} z_{22}^{\star \varepsilon \tau} g_{2}\right\rangle, \\
& G_{23}^{2}=\left\langle\bar{c}_{12}^{*} z_{13}^{\star \varepsilon u 2} g_{1}\right\rangle \text {. }
\end{aligned}
$$


The shear coefficients $A_{i j}$ (with $i=3,4$ and $l=1, \ldots, 9$ ) are given by

$$
\begin{array}{ll}
A_{32}=\left\langle\bar{c}_{44}^{\star} z_{32}^{\varepsilon u}\left(z_{32}^{\varepsilon u}, z_{33}^{\varepsilon u}, z_{35}^{\varepsilon u}\right)\right\rangle, & A_{33}=\left\langle\bar{c}_{44}^{\star} z_{33}^{\varepsilon u}\left(z_{32}^{\varepsilon u}, z_{33}^{\varepsilon u}, z_{35}^{\varepsilon u}\right)\right\rangle, \\
A_{35}=\left\langle\bar{c}_{44}^{\star} z_{35}^{\varepsilon u}\left(z_{32}^{\varepsilon u}, z_{33}^{\varepsilon u}, z_{35}^{\varepsilon u}\right)\right\rangle, & A_{41}=\left\langle\bar{c}_{55}^{\star} z_{41}^{\varepsilon u}\left(z_{41}^{\varepsilon u}, z_{43}^{\varepsilon u}, z_{44}^{\varepsilon u}\right)\right\rangle, \\
A_{43}=\left\langle\bar{c}_{55}^{\star} z_{43}^{\varepsilon u}\left(z_{41}^{\varepsilon u}, z_{43}^{\varepsilon u}, z_{44}^{\varepsilon u}\right)\right\rangle, & A_{44}=\left\langle\bar{c}_{55}^{\star} z_{44}^{\varepsilon u}\left(z_{41}^{\varepsilon u}, z_{43}^{\varepsilon u}, z_{44}^{\varepsilon u}\right)\right\rangle, \\
A_{38}=\left\langle c_{44}^{\star} z_{33}^{\varepsilon \tau}\left(z_{32}^{\varepsilon u}, z_{33}^{\varepsilon u}, z_{35}^{\varepsilon u}\right)\right\rangle, & A_{39}=\left\langle c_{44}^{\star} z_{34}^{\varepsilon \tau}\left(z_{32}^{\varepsilon u}, z_{33}^{\varepsilon u}, z_{35}^{\varepsilon u}\right)\right\rangle, \\
A_{46}=\left\langle c_{55}^{\star} z_{41}^{\varepsilon \tau}\left(z_{41}^{\varepsilon u}, z_{43}^{\varepsilon u}, z_{44}^{\varepsilon u}\right)\right\rangle, & A_{47}=\left\langle c_{55}^{\star} z_{42}^{\varepsilon \tau}\left(z_{41}^{\varepsilon u}, z_{43}^{\varepsilon u}, z_{44}^{\varepsilon u}\right)\right\rangle .
\end{array}
$$

\section{H. Coefficients of the Finite Element Matrices}

$$
\begin{array}{ll}
\left(M_{i j}^{11}, M_{i j}^{14}, M_{i j}^{16}, M_{i j}^{17}\right)=\left(I_{11}^{u u}, I_{14}^{u u}, I_{11}^{\tau u}, I_{21}^{\tau u}\right) S_{11}^{L L}, & \left(M_{i j}^{44}, M_{i j}^{46}, M_{i j}^{47}\right)=\left(I_{44}^{u u}, I_{14}^{\tau u}, I_{24}^{\tau u}\right) S_{11}^{L L}, \\
M_{i r}^{13}=I_{13}^{u u} S_{1 \alpha}^{L H}, & \left(M_{i j}^{55}, M_{i j}^{58}, M_{i j}^{59}\right)=\left(I_{55}^{u u}, I_{35}^{\tau u}, I_{45}^{\tau u}\right) S_{11}^{L L}, \\
\left(M_{i j}^{22}, M_{i j}^{25}, M_{i j}^{28}, M_{i j}^{29}\right)=\left(I_{22}^{u u}, I_{25}^{u u}, I_{32}^{\tau u}, I_{42}^{\tau u}\right) S_{11}^{L L}, & \left(M_{i j}^{66}, M_{i j}^{67}\right)=\left(I_{11}^{\tau \tau}, I_{12}^{\tau \tau}\right) S_{11}^{L L}, \\
M_{i r}^{23}=I_{23}^{u u} S_{1 \beta}^{L H}, & M_{i j}^{77}=I_{22}^{\tau \tau} S_{11}^{L L}, \\
M_{r s}^{33}=I_{33}^{u u} S_{11}^{H H}+I_{33}^{u \alpha} S_{\alpha \alpha}^{H H}+I_{33}^{u \beta} S_{\beta \beta}^{H H}, & \left(M_{i j}^{88}, M_{i j}^{89}\right)=\left(I_{33}^{\tau \tau}, I_{34}^{\tau \tau}\right) S_{11}^{L L}, \\
\left(M_{r j}^{34}, M_{r j}^{36}, M_{r j}^{37}\right)=\left(I_{34}^{u u}, I_{13}^{\tau u}, I_{23}^{\tau u}\right) S_{\alpha 1}^{H L}, & M_{i j}^{99}=I_{44}^{\tau \tau} S_{11}^{L L} . \\
\left(M_{r j}^{35}, M_{r j}^{38}, M_{r j}^{39}\right)=\left(I_{35}^{u u}, I_{33}^{\tau u}, I_{43}^{\tau u}\right) S_{\beta 1}^{H L}, &
\end{array}
$$

$$
\begin{aligned}
& K_{i j}^{11}=\left[A_{11}^{(11)}+A_{21}^{(21)}\right] S_{\alpha \alpha}^{L L}+A_{51}^{(51)} S_{\beta \beta}^{L L}+A_{41}^{(41)} S_{11}^{L L}, \\
& K_{i j}^{12}=\left[A_{12}^{(11)}+A_{22}^{(21)}\right] S_{\alpha \beta}^{L L}+A_{52}^{(51)} S_{\beta \alpha}^{L L}, \\
& K_{i r}^{13}=\left[A_{13}^{1(11)}+A_{23}^{1(21)}\right] S_{\alpha 1}^{L H}+\left[A_{13}^{2(11)}+A_{23}^{2(21)}\right] S_{\alpha \alpha \alpha}^{L H H} \\
& +\left[A_{13}^{3(11)}+A_{23}^{3(21)}\right] S_{\alpha \beta \beta}^{L H H}+A_{53}^{(51)} S_{\beta \alpha \beta}^{L H H}+A_{43}^{(41)} S_{1 \alpha}^{L H}, \\
& K_{i j}^{14}=\left[A_{14}^{(11)}+A_{24}^{(21)}\right] S_{\alpha \alpha}^{L L}+A_{54}^{(51)} S_{\beta \beta}^{L L}+A_{44}^{(41)} S_{11}^{L L}, \\
& K_{i j}^{15}=\left[A_{15}^{(11)}+A_{25}^{(21)}\right] S_{\alpha \beta}^{L L}+A_{55}^{(51)} S_{\beta \alpha}^{L L}, \\
& K_{i j}^{16}=\left[A_{16}^{(11)}+A_{26}^{(21)}\right] S_{\alpha \alpha}^{L L}+A_{56}^{(51)} S_{\beta \beta}^{L L}+A_{46}^{(41)} S_{11}^{L L}, \\
& K_{i j}^{17}=\left[A_{17}^{(11)}+A_{27}^{(21)}\right] S_{\alpha \alpha}^{L L}+A_{57}^{(51)} S_{\beta \beta}^{L L}+A_{47}^{(41)} S_{11}^{L L}, \\
& K_{i j}^{18}=\left[A_{18}^{(11)}+A_{28}^{(21)}\right] S_{\alpha \beta}^{L L}+A_{58}^{(51)} S_{\beta \alpha}^{L L}, \\
& K_{i j}^{19}=\left[A_{19}^{(11)}+A_{29}^{(21)}\right] S_{\alpha \beta}^{L L}+A_{59}^{(51)} S_{\beta \alpha}^{L L}, \\
& K_{i j}^{22}=\left[A_{12}^{(12)}+A_{22}^{(22)}\right] S_{\beta \beta}^{L L}+A_{52}^{(52)} S_{\alpha \alpha}^{L L}+A_{32}^{(32)} S_{11}^{L L}, \\
& K_{i r}^{23}=\left[A_{13}^{1(12)}+A_{23}^{1(22)}\right] S_{\beta 1}^{L H}+\left[A_{13}^{2(12)}+A_{23}^{2(22)}\right] S_{\beta \alpha \alpha}^{L H H} \\
& +\left[A_{13}^{3(12)}+A_{23}^{3(22)}\right] S_{\beta \beta \beta}^{L H H}+A_{53}^{(52)} S_{\alpha \alpha \beta}^{L H H}+A_{33}^{(32)} S_{1 \beta}^{L H}, \\
& K_{i j}^{24}=\left[A_{14}^{(12)}+A_{24}^{(22)}\right] S_{\beta \alpha}^{L L}+A_{54}^{(52)} S_{\alpha \beta}^{L L}, \\
& K_{i j}^{25}=\left[A_{15}^{(12)}+A_{25}^{(22)}\right] S_{\beta \beta}^{L L}+A_{55}^{(52)} S_{\alpha \alpha}^{L L}+A_{35}^{(32)} S_{11}^{L L}, \\
& K_{i j}^{26}=\left[A_{16}^{(12)}+A_{26}^{(22)}\right] S_{\beta \alpha}^{L L}+A_{56}^{(52)} S_{\alpha \beta}^{L L}, \\
& K_{i j}^{27}=\left[A_{17}^{(12)}+A_{27}^{(22)}\right] S_{\beta \alpha}^{L L}+A_{57}^{(52)} S_{\alpha \beta}^{L L}, \\
& K_{i j}^{28}=\left[A_{18}^{(12)}+A_{28}^{(22)}\right] S_{\beta \beta}^{L L}+A_{58}^{(52)} S_{\alpha \alpha}^{L L}+A_{38}^{(32)} S_{11}^{L L}, \\
& K_{i j}^{29}=\left[A_{19}^{(12)}+A_{29}^{(22)}\right] S_{\beta \beta}^{L L}+A_{59}^{(52)} S_{\alpha \alpha}^{L L}+A_{39}^{(32)} S_{11}^{L L},
\end{aligned}
$$




$$
\begin{aligned}
& K_{r s}^{33}=\left[B_{13}^{1(131)}+B_{23}^{1(231)}\right] S_{11}^{H H}+\left[B_{13}^{2(131)}+B_{23}^{2(231)}\right] S_{1 \alpha \alpha}^{H H H}+\left[B_{13}^{3(131)}+B_{23}^{3(231)}\right] S_{1 \beta \beta}^{H H H} \\
& +B_{13}^{1(132)} S_{\alpha \alpha 1}^{H H H}+B_{13}^{2(132)} S_{\alpha \alpha \alpha \alpha}^{H H H H}+B_{13}^{3(132)} S_{\alpha \alpha \beta \beta}^{H H H}+B_{23}^{1(232)} S_{\beta \beta 1}^{H H H}+B_{23}^{2(232)} S_{\beta \beta \alpha \alpha}^{H H H} \\
& +B_{23}^{3(232)} S_{\beta \beta \beta \beta}^{H H H}+B_{53}^{(53)} S_{\alpha \beta \alpha \beta}^{H H H H}+A_{43}^{(43)} S_{\alpha \alpha}^{H H}+A_{33}^{(33)} S_{\beta \beta}^{H H}, \\
& K_{r j}^{34}=\left[B_{14}^{(131)}+B_{24}^{(231)}\right] S_{1 \alpha}^{H L}+B_{14}^{(132)} S_{\alpha \alpha \alpha}^{H H L}+B_{24}^{(232)} S_{\beta \beta \alpha}^{H H L}+B_{54}^{(53)} S_{\alpha \beta \beta}^{H H L}+A_{44}^{(43)} S_{\alpha 1}^{H L}, \\
& K_{r j}^{35}=\left[B_{15}^{(131)}+B_{25}^{(231)}\right] S_{1 \beta}^{H L}+B_{15}^{(132)} S_{\alpha \alpha \beta}^{H H L}+B_{25}^{(232)} S_{\beta \beta \beta}^{H H L}+B_{55}^{(53)} S_{\alpha \beta \alpha}^{H H L}+A_{35}^{(33)} S_{\beta 1}^{H L}, \\
& K_{r j}^{36}=\left[B_{16}^{(131)}+B_{26}^{(231)}\right] S_{1 \alpha}^{H L}+B_{16}^{(132)} S_{\alpha \alpha \alpha}^{H H L}+B_{26}^{(232)} S_{\beta \beta \alpha}^{H H L}+B_{56}^{(53)} S_{\alpha \beta \beta}^{H H L}+A_{46}^{(43)} S_{\alpha 1}^{H L}, \\
& K_{r j}^{37}=\left[B_{17}^{(131)}+B_{27}^{(231)}\right] S_{1 \alpha}^{H L}+B_{17}^{(132)} S_{\alpha \alpha \alpha}^{H H L}+B_{27}^{(232)} S_{\beta \beta \alpha}^{H H L}+B_{57}^{(53)} S_{\alpha \beta \beta}^{H H L}+A_{47}^{(43)} S_{\alpha 1}^{H L}, \\
& K_{r j}^{38}=\left[B_{18}^{(131)}+B_{28}^{(231)}\right] S_{1 \beta}^{H L}+B_{18}^{(132)} S_{\alpha \alpha \beta}^{H H L}+B_{28}^{(232)} S_{\beta \beta \beta}^{H H L}+B_{58}^{(53)} S_{\alpha \beta \alpha}^{H H L}+A_{38}^{(33)} S_{\beta 1}^{H L}, \\
& K_{r j}^{39}=\left[B_{19}^{(131)}+B_{29}^{(231)}\right] S_{1 \beta}^{H L}+B_{19}^{(132)} S_{\alpha \alpha \beta}^{H H L}+B_{29}^{(232)} S_{\beta \beta \beta}^{H H L}+B_{59}^{(53)} S_{\alpha \beta \alpha}^{H H L}+A_{39}^{(33)} S_{\beta 1}^{H L}, \\
& K_{i j}^{44}=\left[B_{14}^{(14)}+B_{24}^{(24)}\right] S_{\alpha \alpha}^{L L}+B_{54}^{(54)} S_{\beta \beta}^{L L}+A_{44}^{(44)} S_{11}^{L L}, \\
& K_{i j}^{45}=\left[B_{15}^{(14)}+B_{25}^{(24)}\right] S_{\alpha \beta}^{L L}+B_{55}^{(54)} S_{\beta \alpha}^{L L}, \\
& K_{i j}^{46}=\left[B_{16}^{(14)}+B_{26}^{(24)}\right] S_{\alpha \alpha}^{L L}+B_{56}^{(54)} S_{\beta \beta}^{L L}+A_{46}^{(44)} S_{11}^{L L}, \\
& K_{i j}^{47}=\left[B_{17}^{(14)}+B_{27}^{(24)}\right] S_{\alpha \alpha}^{L L}+B_{57}^{(54)} S_{\beta \beta}^{L L}+A_{47}^{(44)} S_{11}^{L L}, \\
& K_{i j}^{48}=\left[B_{18}^{(14)}+B_{28}^{(24)}\right] S_{\alpha \beta}^{L L}+B_{58}^{(54)} S_{\beta \alpha}^{L L}, \\
& K_{i j}^{49}=\left[B_{19}^{(14)}+B_{29}^{(24)}\right] S_{\alpha \beta}^{L L}+B_{59}^{(54)} S_{\beta \alpha}^{L L}, \\
& K_{i j}^{55}=\left[B_{15}^{(15)}+B_{25}^{(25)}\right] S_{\beta \beta}^{L L}+B_{55}^{(55)} S_{\alpha \alpha}^{L L}+A_{35}^{(35)} S_{11}^{L L}, \\
& K_{i j}^{56}=\left[B_{16}^{(15)}+B_{26}^{(25)}\right] S_{\beta \alpha}^{L L}+B_{56}^{(55)} S_{\alpha \beta}^{L L}, \\
& K_{i j}^{57}=\left[B_{17}^{(15)}+B_{27}^{(25)}\right] S_{\beta \alpha}^{L L}+B_{57}^{(55)} S_{\alpha \beta}^{L L}, \\
& K_{i j}^{58}=\left[B_{18}^{(15)}+B_{28}^{(25)}\right] S_{\beta \beta}^{L L}+B_{58}^{(55)} S_{\alpha \alpha}^{L L}+A_{38}^{(35)} S_{11}^{L L}, \\
& K_{i j}^{59}=\left[B_{19}^{(15)}+B_{29}^{(25)}\right] S_{\beta \beta}^{L L}+B_{59}^{(55)} S_{\alpha \alpha}^{L L}+A_{39}^{(35)} S_{11}^{L L}, \\
& K_{i j}^{66}=\left[C_{16}^{(11)}+C_{26}^{(21)}\right] S_{\alpha \alpha}^{L L}+C_{56}^{(51)} S_{\beta \beta}^{L L}+C_{46}^{(41)} S_{11}^{L L}, \\
& K_{i j}^{67}=\left[C_{17}^{(11)}+C_{27}^{(21)}\right] S_{\alpha \alpha}^{L L}+C_{57}^{(51)} S_{\beta \beta}^{L L}+C_{47}^{(41)} S_{11}^{L L}, \\
& K_{i j}^{68}=\left[C_{18}^{(11)}+C_{28}^{(21)}\right] S_{\alpha \beta}^{L L}+C_{58}^{(51)} S_{\beta \alpha}^{L L}, \\
& K_{i j}^{69}=\left[C_{19}^{(11)}+C_{29}^{(21)}\right] S_{\alpha \beta}^{L L}+C_{59}^{(51)} S_{\beta \alpha}^{L L}, \\
& K_{i j}^{77}=\left[C_{17}^{(12)}+C_{27}^{(22)}\right] S_{\alpha \alpha}^{L L}+C_{57}^{(52)} S_{\beta \beta}^{L L}+C_{47}^{(42)} S_{11}^{L L}, \\
& K_{i j}^{78}=\left[C_{18}^{(12)}+C_{28}^{(22)}\right] S_{\alpha \beta}^{L L}+C_{58}^{(52)} S_{\beta \alpha}^{L L}, \\
& K_{i j}^{79}=\left[C_{19}^{(12)}+C_{29}^{(22)}\right] S_{\alpha \beta}^{L L}+C_{59}^{(52)} S_{\beta \alpha}^{L L}, \\
& K_{i j}^{88}=\left[C_{18}^{(13)}+C_{28}^{(23)}\right] S_{\beta \beta}^{L L}+C_{58}^{(53)} S_{\alpha \alpha}^{L L}+C_{38}^{(33)} S_{11}^{L L}, \\
& K_{i j}^{89}=\left[C_{19}^{(13)}+C_{29}^{(23)}\right] S_{\beta \beta}^{L L}+C_{59}^{(53)} S_{\alpha \alpha}^{L L}+C_{39}^{(33)} S_{11}^{L L}, \\
& K_{i j}^{99}=\left[C_{19}^{(14)}+C_{29}^{(24)}\right] S_{\beta \beta}^{L L}+C_{59}^{(54)} S_{\alpha \alpha}^{L L}+C_{39}^{(34)} S_{11}^{L L},
\end{aligned}
$$




$$
\begin{aligned}
& F_{i}^{1}=\oint_{\Gamma_{\alpha}^{e}} L_{i}^{e} \hat{N}_{\alpha \beta}^{11} d \alpha+\oint_{\Gamma_{\beta}^{e}} L_{i}^{e} \hat{N}_{\alpha \alpha}^{11} d \beta, \quad F_{i}^{2}=\oint_{\Gamma_{\alpha}^{e}} L_{i}^{e} \hat{N}_{\beta \beta}^{22} d \alpha+\oint_{\Gamma_{\beta}^{e}} L_{i}^{e} \hat{N}_{\alpha \beta}^{22} d \beta, \\
& F_{i}^{3}=\int_{\Omega_{0}^{e}} H_{r}^{e} Z d \alpha d \beta+\oint_{\Gamma_{\alpha}^{e}}\left(\frac{\partial H_{r}^{e}}{\partial \beta} \hat{M}_{\beta \beta}^{23}+\frac{\partial H_{r}^{e}}{\partial \alpha} \hat{M}_{\alpha \beta}^{13}+H_{r}^{e} \hat{Q}_{\beta z}^{33}\right) d \alpha \\
& +\oint_{\Gamma_{\beta}^{e}}\left(\frac{\partial H_{r}^{e}}{\partial \alpha} \hat{M}_{\alpha \alpha}^{13}+\frac{\partial H_{r}^{e}}{\partial \beta} \hat{M}_{\alpha \alpha}^{23}+H_{r}^{e} \hat{Q}_{z \alpha}^{33}\right) d \beta \\
& F_{i}^{4}=\oint_{\Gamma_{\alpha}^{e}} L_{i}^{e} \hat{M}_{\alpha \beta}^{14} d \alpha+\oint_{\Gamma_{\beta}^{e}} L_{i}^{e} \hat{M}_{\alpha \alpha}^{14} d \beta, \quad F_{i}^{5}=\oint_{\Gamma_{\alpha}^{e}} L_{i}^{e} \hat{M}_{\beta \beta}^{25} d \alpha+\oint_{\Gamma_{\beta}^{e}} L_{i}^{e} \hat{M}_{\alpha \beta}^{25} d \beta, \\
& F_{i}^{6}=\oint_{\Gamma_{\alpha}^{e}} L_{i}^{e} \hat{T}_{\beta \alpha}^{11} d \alpha+\oint_{\Gamma_{\beta}^{e}} L_{i}^{e} \hat{T}_{\alpha \alpha}^{11} d \beta, \quad F_{i}^{7}=\oint_{\Gamma_{\alpha}^{e}} L_{i}^{e} \hat{T}_{\beta \alpha}^{12} d \alpha+\oint_{\Gamma_{\beta}^{e}} L_{i}^{e} \hat{T}_{\alpha \alpha}^{12} d \beta, \\
& F_{i}^{8}=\oint_{\Gamma_{\alpha}^{e}} L_{i}^{e} \hat{T}_{\beta \beta}^{23} d \alpha+\oint_{\Gamma_{\beta}^{e}} L_{i}^{e} \hat{T}_{\alpha \beta}^{23} d \beta \\
& K_{i \phi}^{1}=\int_{\Omega_{0}^{e}} \frac{\partial L_{i}^{e}}{\partial \alpha}\left(P_{\alpha \alpha}^{\star 11}+P_{\beta \beta}^{\star 21}\right) d \alpha d \beta, \quad K_{i \phi}^{2}=\int_{\Omega_{0}^{e}} \frac{\partial L_{i}^{e}}{\partial \beta}\left(P_{\alpha \alpha}^{\star 12}+P_{\beta \beta}^{\star 22}\right) d \alpha d \beta, \\
& K_{r \phi}^{3}=\int_{\Omega_{0}^{e}}\left[H_{r}^{e}\left(P_{\alpha \alpha}^{\star 131}+P_{\beta \beta}^{\star 231}\right)+\frac{\partial^{2} H_{r}^{e}}{\partial \alpha^{2}} P_{\alpha \alpha}^{\star 132}+\frac{\partial^{2} H_{r}^{e}}{\partial \beta^{2}} P_{\beta \beta}^{\star 232}\right] d \alpha d \beta, \\
& K_{i \phi}^{4}=\int_{\Omega_{0}^{e}} \frac{\partial L_{i}^{e}}{\partial \alpha}\left(P_{\alpha \alpha}^{\star 14}+P_{\beta \beta}^{\star 24}\right) d \alpha d \beta, \quad K_{i \phi}^{5}=\int_{\Omega_{0}^{e}} \frac{\partial L_{i}^{e}}{\partial \beta}\left(P_{\alpha \alpha}^{\star 15}+P_{\beta \beta}^{\star 25}\right) d \alpha d \beta, \\
& K_{i \phi}^{6}=\int_{\Omega_{0}^{e}} \frac{\partial L_{i}^{e}}{\partial \alpha}\left(R_{\alpha \alpha}^{\star 11}+R_{\beta \beta}^{\star 21}\right) d \alpha d \beta, \quad K_{i \phi}^{7}=\int_{\Omega_{0}^{e}} \frac{\partial L_{i}^{e}}{\partial \alpha}\left(R_{\alpha \alpha}^{\star 12}+R_{\beta \beta}^{\star 22}\right) d \alpha d \beta, \\
& K_{i \phi}^{8}=\int_{\Omega_{0}^{e}} \frac{\partial L_{i}^{e}}{\partial \beta}\left(R_{\alpha \alpha}^{\star 13}+R_{\beta \beta}^{\star 23}\right) d \alpha d \beta, \quad K_{i \phi}^{9}=\int_{\Omega_{0}^{e}} \frac{\partial L_{i}^{e}}{\partial \beta}\left(R_{\alpha \alpha}^{\star 14}+R_{\beta \beta}^{\star 24}\right) d \alpha d \beta, \\
& K_{\phi \phi}=\int_{\Omega_{0}^{e}} S_{\phi \phi} d \alpha d \beta, \quad Q_{\phi}=\int_{\Omega_{0}^{e}} \tau d \alpha d \beta .
\end{aligned}
$$

where the zero- (1), first- $(\alpha$ or $\beta)$ and second-order $(\alpha \alpha, \beta \beta$ or crossed $\alpha \beta)$ derivatives, taken as subscripts of $S$, of the Lagrange $(L)$ and Hermite $(H)$ interpolation functions, superscripts of $S$, where defined as follows (for the sake of brevity only a few terms are presented since the rest are obvious from the following relations),

$$
\begin{gathered}
S_{11}^{L L}=\int_{\Omega_{0}^{e}} L_{i}^{e} L_{j}^{e} d \alpha d \beta, \quad S_{1 \alpha}^{L H}=\int_{\Omega_{0}^{e}} L_{i}^{e} \frac{\partial H_{r}^{e}}{\partial \alpha} d \alpha d \beta, \quad S_{\beta \beta}^{L L}=\int_{\Omega_{0}^{e}} \frac{\partial L_{i}^{e}}{\partial \beta} \frac{\partial L_{j}^{e}}{\partial \beta} d \alpha d \beta, \\
S_{\beta \alpha \beta}^{L H H}=\int_{\Omega_{0}^{e}} \frac{\partial L_{i}^{e}}{\partial \beta} \frac{\partial^{2} H_{r}^{e}}{\partial \alpha \partial \beta} d \alpha d \beta, \quad S_{\alpha \beta \alpha \beta}^{H H H H}=\int_{\Omega_{0}^{e}} \frac{\partial^{2} H_{r}^{e}}{\partial \alpha \partial \beta} \frac{\partial^{2} H_{r}^{e}}{\partial \alpha \partial \beta} d \alpha d \beta .
\end{gathered}
$$

\section{Acknowledgments}

The financial support given by Fundação para a Ciência e a Tecnologia of the Ministério da Ciência e da Tecnologia of Portugal under grant POSI SFRH/BD/13255/2003, Fundação Calouste Gulbenkian and Fundação Luso-Americana para o Desenvolvimento are gratefully acknowledged.

\section{References}

${ }^{1}$ Kraus, H., Thin Elastic Shells, John Wiley \& Sons, New York, 1967.

${ }^{2}$ Love, A. E. H., Treatise on the Mathematical Theory of Elasticity, Dover Publications, New York, 4th ed., 1944.

${ }^{3}$ Naghdi, P. M., "A survey of recent progress in the theory of elastic shells," Applied Mechanics Reviews, Vol. 9, No. 9, 1956, pp. 365-368.

${ }^{4}$ Leissa, A. W., Vibration of Shells, Acoustical Society of America, 1993.

${ }^{5}$ Yang, H. T. Y., Saigal, S., Masud, A., and Kapania, R. K., "A survey of recent shell finite elements," International Journal for Numerical Methods in Engineering, Vol. 47, No. 1-3, 2000, pp. 101-127. 
${ }^{6}$ Reddy, J. N., Mechanics of Laminated Composite Plates and Shells: Theory and Analysis, CRC Press, Boca Raton, Florida, 2nd ed., 2004.

${ }^{7}$ Koiter, W. T., "A consistent first approximation in the general theory of thin elastic shells," Proceedings of the Symposium on Theory of Thin Elastic Shells (IUTAM), Delft, August 24-28, 1959, North Holland, Delft, 1960, pp. 12-33.

${ }^{8}$ Carrera, E., "A study of transverse normal stress effect on vibration of multilayered plates and shells," Journal of Sound and Vibration, Vol. 225, No. 5, 1999, pp. 803-829.

${ }^{9}$ Srinivas, S., Rao, C. V. J., and Rao, A. K., "Flexural vibration of rectangular plates," Journal of Applied Mechanics, Vol. 23, 1970, pp. 430-436.

${ }^{10}$ Srinivas, S., "Analysis of Laminated, Composite, Circular Cylindrical Shells with General Boundary Conditions," NASA TR R-412, 1974.

${ }^{11}$ Pagano, N. J. and Reddy, J. N., Mechanics of Composite Materials: Selected Works of Nicholas J. Pagano, Solid Mechanics and its Applications, vol. 34, Kluwer Academic Publishers, Dordrecht, 1994.

${ }^{12}$ Ren, J. G., "Exact Solutions for Laminated Cylindrical Shells in Cylindrical Bending," Composites Science and Technology, Vol. 29, No. 3, 1987, pp. 169-187.

${ }^{13}$ Varadan, T. K. and Bhaskar, K., "Bending of laminated orthotropic cylindrical shells - An elasticity approach," Composite Structures, Vol. 17, No. 2, 1991, pp. 141-156.

${ }^{14}$ Bhaskar, K. and Varadan, T. K., "Benchmark elasticity solution for locally loaded laminated orthotropic cylindrical shells," AIAA Journal, Vol. 32, No. 3, 1994, pp. 627-632.

${ }^{15}$ Soldatos, K. P., "Review of three dimensional dynamic analyses of circular cylinders and cylindrical shells," Applied Mechanics Reviews, Vol. 47, No. 10, 1994, pp. 501-516.

${ }^{16}$ Carrera, E., "C $C_{z}^{0}$ Requirements - models for the two dimensional analysis of multilayered structures," Composite Structures, Vol. 37, No. 3-4, 1997, pp. 373-383.

${ }^{17}$ Reddy, J. N. and Liu, C. F., "A Higher-Order Shear Deformation Theory of Laminated Elastic Shells," International Journal of Engineering Science, Vol. 23, No. 3, 1985, pp. 319-330.

${ }^{18}$ Reddy, J. N., "A General Nonlinear Third-Order Theory of Plates with Moderate Thickness," International Journal of Non-Linear Mechanics, Vol. 25, No. 6, 1990, pp. 677-686.

${ }^{19}$ Garção, J. E. S., Soares, C. M. M., Soares, C. A. M., and Reddy, J. N., "Analysis of Laminated Adaptive Plate Structures Using Layerwise Finite Element Models," Computers and Structures, Vol. 82, No. 23-26, 2004, pp. 1939-1959.

${ }^{20}$ Lage, R. G., Soares, C. M. M., Soares, C. A. M., and Reddy, J. N., "Analysis of Adaptive Plate Structures by Mixed Layerwise Finite Elements," Composite Structures, Vol. 66, No. 1-4, 2004, pp. 269-276.

${ }^{21}$ Carrera, E., "Historical Review of Zig-Zag Theories for Multilayered Plates and Shells," Applied Mechanics Reviews, Vol. 56, No. 3, 2003, pp. 287.

${ }^{22}$ Lekhnitskii, S. G., Anisotropic Plates, Translated from the Russian Edition by S. W. Tsai and T. Cheron, Gordon and Breach, New York, 2nd ed., 1968.

${ }^{23}$ Ambartsumyan, S. A., Theory of Anisotropic Plates: Strength, Stability, and Vibrations, Hemisphere, 2nd ed., 1991.

${ }^{24}$ Ambartsumian, S. A., Fragments of the Theory of Anisotropic Shells, World Scientific Publishing, Singapure, 1991.

${ }^{25}$ Ashton, J. E. and Whitney, J. M., Theory of Laminated Plates, Technomic Publishing, Stamford, 1970.

${ }^{26}$ Rath, B. K. and Das, Y. C., "Vibration of Layered Shells," Journal of Sound and Vibration, Vol. 28, No. 4, 1973, pp. $737-757$.

${ }^{27}$ Cho, M. and Parmerter, R. R., "Efficient higher order composite plate theory for general lamination configurations," AIAA Journal, Vol. 31, No. 7, 1993, pp. 1299-1306.

${ }^{28}$ Beakou, A. and Touratier, M., "Rectangular finite element for analysing composite multilayered shallow shells in statics, vibration and buckling," International Journal for Numerical Methods in Engineering, Vol. 36, No. 4, 1993, pp. $627-653$.

${ }^{29}$ Soldatos, K. P. and Timarci, T., "A Unified Formulation of Laminated Composite, Shear-Deformable, Five-Degrees-ofFreedom Cylindrical-Shell Theories," Composite Structures, Vol. 25, No. 1-4, 1993, pp. 165-171.

${ }^{30}$ Reissner, E., "On a Certain Mixed Variational Theorem and a Proposed Application," International Journal for Numerical Methods in Engineering, Vol. 20, No. 7, 1984, pp. 1366-1368.

${ }^{31}$ Murakami, H., "Laminated Composite Plate Theory with Improved In-Plane Responses," Journal of Applied Mechanics, Vol. 53, No. 3, 1986, pp. 661-666.

${ }^{32}$ Carrera, E., "On the use of the Murakami's zig-zag function in the modeling of layered plates and shells," Computers and Structures, Vol. 82, No. 7-8, 2004, pp. 541-554.

${ }^{33}$ Carrera, E., "Theories and Finite Elements for Multilayered Plates and Shells: A Unified Compact Formulation with Numerical Assessment and Benchmarking," Archives of Computational Methods in Engineering, Vol. 10, No. 3, 2003, pp. 215296.

${ }^{34}$ Noor, A. K. and Burton, W. S., "Assessment of Shear Deformation Theories for Multilayered Composite Plates," Applied Mechanics Reviews, Vol. 42, No. 1, 1989, pp. 1-13.

${ }^{35}$ Noor, A. K., Burton, W. S., and Bert, C. W., "Computational Models for Sandwich Panels and Shells," Applied Mechanics Reviews, Vol. 49, No. 3, 1996, pp. 155-199.

${ }^{36}$ Ghugal, Y. M. and Shimpi, R. P., "A review of refined shear deformation theories for isotropic and anisotropic laminated beams," Journal of Reinforced Plastics and Composites, Vol. 20, No. 3, 2001, pp. 255-272.

${ }^{37}$ Ghugal, Y. M. and Shimpi, R. P., "A review of refined shear deformation theories of isotropic and anisotropic laminated plates," Journal of Reinforced Plastics and Composites, Vol. 21, No. 9, 2002, pp. 775-813.

${ }^{38}$ Carrera, E., "Theories and finite elements for multilayered, anisotropic, composite plates and shells," Archives of Computational Methods in Engineering, Vol. 9, No. 2, 2002, pp. 87-140.

${ }^{39}$ Reddy, J. N. and Arciniega, R. A., "Shear Deformation Plate and Shell Theories: From Stavsky to Present," Mechanics of Advanced Materials and Structures, Vol. 11, No. 6, 2004, pp. 535-582. 


\begin{abstract}
${ }^{40}$ Krommer, M. and Irschik, H., "On the influence of the electric field on free transverse vibrations of smart beams," Smart Materials and Structures, Vol. 8, No. 3, 1999, pp. 401-410.

${ }^{41}$ Krommer, M. and Irschik, H., "A Reissner-Mindlin-Type Plate Theory Including the Direct Piezoelectric and the Pyroelectric Effect," Acta Mechanica, Vol. 141, No. 1-2, 2000, pp. 51-69.

${ }^{42}$ Vasques, C. M. A. and Rodrigues, J. D., "Coupled Three-Layered Analysis of Smart Piezoelectric Beams with Different Electric Boundary Conditions," International Journal for Numerical Methods in Engineering, Vol. 62, No. 11, 2005, pp. 14881518.
\end{abstract}

${ }^{43}$ Tiersten, H. F., Linear Piezoelectric Plate Vibrations, Plenum Press, New York, 1969.

${ }^{44}$ Tzou, H. S., Piezoelectric Shells: Distributed Sensing and Control of Continua, Kluwer Academic Publishers, Dordrecht, Netherlands, 1993.

${ }^{45}$ Rogacheva, N. N., The Theory of Piezoelectric Shells and Plates, CRC Press, Boca Raton, Florida, 1994.

${ }^{46} \mathrm{Baz}$, A., "Active Constrained Layer Damping," Proceedings of Damping' 93, Vol. 3, San Francisco, CA, 1993, pp. IBB $1-23$.

${ }^{47}$ Moreira, R. A. and Rodrigues, J. D., "The Modelisation of Constrained Damping Layer Treatment Using the Finite Element Method: Spatial and Viscoelastic Behavior," Proceedings of the International Conference on Structural Dynamics Modelling: Test, Analysis Correlation and Validation, Madeira, Portugal, 2002.

${ }^{48}$ Moreira, R. and Rodrigues, J., "Constrained Damping Layer Treatments: Finite Element Modeling," Journal of Vibration and Control, Vol. 10, No. 4, 2004, pp. 575-595.

${ }^{49}$ Johnson, C. D., Kienholz, D. A., and Rogers, L. C., "Finite element prediction of damping in beams with constrained viscoelastic layers," Shock and Vibration Bulletin, No. 1, 1980, pp. 71-81.

${ }^{50}$ Trindade, M. A., Benjeddou, A., and Ohayon, R., "Modeling of Frequency-Dependent Viscoelastic Materials for ActivePassive Vibration Damping," Journal of Vibration and Acoustics, Vol. 122, No. 2, 2000, pp. 169-174.

${ }^{51}$ Johnson, A. R., "Modeling viscoelastic materials using internal variables," Shock and Vibration Digest, Vol. 31, No. 2, 1999, pp. 91-100.

${ }^{52}$ Golla, D. F. and Hughes, P. C., "Dynamics of Viscoelastic Structures - A Time-Domain, Finite Element Formulation," Jounal of Applied Mechanics, Vol. 52, No. 12, 1985, pp. 897-906.

${ }^{53}$ McTavish, D. J. and Hughes, P. C., "Modeling of Linear Viscoelastic Space Structures," Journal of Vibration and Acoustics, Vol. 115, No. 1, 1993, pp. 103-110.

${ }^{54}$ Lesieutre, G. A. and Bianchini, E., "Time Domain Modeling of Linear Viscoelasticity Using Anelastic Displacement Fields," Journal of Vibration and Acoustics, Vol. 117, No. 4, 1995, pp. 424-430.

${ }^{55}$ Lesieutre, G. A., Bianchini, E., and Maiani, A., "Finite Element Modeling of One-Dimensional Viscoelastic Structures Using Anelastic Displacement Fields," Journal of Guidance Control and Dynamics, Vol. 19, No. 3, 1996, pp. 520-527.

${ }^{56}$ Yiu, Y., "Finite element analysis of structures with classical viscoelastic materials," 34 th AIAA/ASME/ASCE/ AHS/ASC Structures, Structural Dynamics and Materials Conference, Vol. 4, La Jolla, CA, USA, 1993, pp. 2110-2119.

${ }^{57}$ Silva, L. A., Internal Variable and Temperature Modeling Behavior of Viscoelastic Structures - A Control Analysis, $\mathrm{PhD}$ thesis, Virginia Tech, VA, USA, 2003.

${ }^{58}$ Bagley, R. L. and Torvik, P. J., "Fractional Calculus - A Different Approach to The Analysis of Viscoelastically Damped Structures," AIAA Journal, Vol. 21, No. 5, 1983, pp. 741-748.

${ }^{59}$ Bagley, R. L. and Torvik, P. J., "Fractional Calculus in the Transient Analysis of Viscoelastically Damped Structures," AIAA Journal, Vol. 23, No. 6, 1985, pp. 918-925.

${ }^{60}$ Adhikari, S. and Woodhouse, J., "Quantification of non-viscous damping in discrete linear systems," Journal of Sound and Vibration, Vol. 260, No. 3, 2003, pp. 499-518.

${ }^{61}$ Brackbill, C. R., Lesieutre, G. A., Smith, E. C., and Govindswamy, K., "Thermomechanical modeling of elastomeric materials," Smart Materials \&s Structures, Vol. 5, No. 5, 1996, pp. 529-539.

${ }^{62}$ Lesieutre, G. A. and Govindswamy, K., "Finite element modeling of frequency dependent and temperature-dependent dynamic behavior of viscoelastic materials in simple shear," International Journal of Solids and Structures, Vol. 33, No. 3, 1996, pp. 419-432.

${ }^{63}$ Baz, A., "Robust Control of Active Constrained Layer Damping," Journal of Sound and Vibration, Vol. 211, No. 3, 1998, pp. 467-480.

${ }^{64}$ Friswell, M. I. and Inman, D. J., "Hybrid damping treatments in thermal environments," Smart Materials and Structures, edited by G. Tomlinson and W. Bullough, IOP Publishing, Bristol, UK, 1998, pp. 667-674.

${ }^{65}$ Trindade, M. A., Benjeddou, A., and Ohayon, R., "Finite Element Analysis of Frequency- and Temperature-Dependent Hybrid Active-Passive Vibration Damping," Revue Européenne des Éléments Finis, Vol. 9, No. 1-3, 2000, pp. 89-111.

${ }^{66}$ Silva, L. A., Austin, E. M., and Inman, D. J., "Time-Varying Controller for Temperature-Dependent Viscoelasticity," Journal of Vibration and Acoustics, Vol. 127, No. 3, 2005, pp. 215-222.

${ }^{67}$ Pradeep, V. and Ganesan, N., "Vibration behavior of ACLD treated beams under thermal environment," Journal of Sound and Vibration, Vol. 292, No. 3-5, 2006, pp. 1036-1045.

${ }^{68}$ Vasques, C. M. A., Moreira, R. A. S., and Rodrigues, J. D., "Experimental Identification of GHM and ADF Parameters for Viscoelastic Damping Modeling," Proceedings of the III European Conference on Computational Mechanics: Solids, Structures and Coupled Problems in Engineering, edited by C. A. Mota Soares et al., CD-ROM Edition, Springer, 5-8 June, Lisbon, Portugal, 2006 (to be published).

${ }^{69}$ Park, C. H. and Baz, A., "Vibration Damping and Control Using Active Constrained Layer Damping: A Survey," The Shock and Vibration Digest, Vol. 31, No. 5, 1999, pp. 355-364.

${ }^{70}$ Stanway, R., Rongong, J. A., and Sims, N. D., "Active constrained-layer damping: A state-of-the-art review," Proceedings of the Institution of Mechanical Engineers. Part I: Journal of Systems and Control Engineering, Vol. 217, No. 6, 2003, pp. $437-456$. 
${ }^{71}$ Benjeddou, A., "Advances in Piezoelectric Finite Element Modelling of Adaptive Structural Elements: A survey," Computers and Structures, Vol. 76, No. 1-3, 2000, pp. 347-363.

${ }^{72}$ Benjeddou, A., "Advances in Hybrid Active-Passive Vibration and Noise Control Via Piezoelectric and Viscoelastic Constrained Layer Treatments," Journal of Vibration and Control, Vol. 7, No. 4, 2001, pp. 565-602.

${ }^{73}$ Trindade, M. A. and Benjeddou, A., "Hybrid active-passive damping treatments using viscoelastic and piezoelectric materials: Review and assessment," Journal of Vibration and Control, Vol. 8, No. 6, 2002, pp. 699-745.

${ }^{74}$ Inman, D. J., Vibration: With Control, Measurement, and Stability, Prentice Hall, Englewood Cliffs, 1989.

${ }^{75}$ Meirovitch, L., Dynamics and Control of Structures, John Wiley \& Sons, New York, 1990.

${ }^{76}$ Nelson, P. A. and Elliott, S. J., Active Control of Sound, Academic Press, London, 1993.

${ }^{77}$ Fuller, C. R., Elliott, S. J., and Nelson, P. A., Active Control of Vibration, Academic Press, London, 1996.

${ }^{78}$ Hansen, C. H. and Snyder, S. D., Active Control of Noise and Vibration, E \& FN Spon, London, 1997.

${ }^{79}$ Clark, R. L., Saunders, W. R., and Gibbs, G. P., Adaptive Structures: Dynamics and Control, John Wiley \& Sons, New York, 1998.

${ }^{80}$ Elliott, S. J., Signal Processing for Active Control, Signal processing and its applications, Academic Press, San Diego, CA, 2001.

${ }^{81}$ Preumont, A., Vibration Control of Active Structures: An Introduction, Kluwer Academic Publishers, Dordrecht, 2nd ed., 2002.

${ }^{82}$ Alkhatib, R. and Golnaraghi, M. F., "Active structural vibration control: A review," Shock and Vibration Digest, Vol. 35, No. 5, 2003, pp. 367-383.

${ }^{83}$ Trindade, M. A., Benjeddou, A., and Ohayon, R., "Piezoelectric active vibration control of damped sandwich beams," Journal of Sound and Vibration, Vol. 246, No. 4, 2001, pp. 653-677.

${ }^{84}$ Baz, A., "Active Constrained Layer Damping of Thin Cylindrical Shells," Journal of Sound and Vibration, Vol. 240, No. 5, 2001, pp. 921-935.

${ }^{85}$ Ray, M. C. and Reddy, J. N., "Optimal control of thin circular cylindrical laminated composite shells using active constrained layer damping treatment," Smart Materials and Structures, Vol. 13, No. 1, 2004, pp. 64.

${ }^{86} \mathrm{Baz}, \mathrm{A}$. and Chen, T., "Control of axi-symmetric vibrations of cylindrical shells using active constrained layer damping," Thin-Walled Structures, Vol. 36, No. 1, 2000, pp. 1-20.

${ }^{87}$ Gandhi, F. and Munsky, B., "Comparison of Damping Augmentation Mechanisms with Position and Velocity Feedback in Active Constrained Layer Treatments," Journal of Intelligent Material Systems and Structures, Vol. 13, No. 5, 2002, pp. 317326.

${ }^{88}$ Vasques, C. M. A. and Rodrigues, J. D., "Active Vibration Control of Smart Piezoelectric Beams: Comparison of Classical and Optimal Feedback Control Strategies," Computers and Structures, 2005 (to be published).

${ }^{89}$ Poh, S., Baz, A., and Balachandran, B., "Experimental adaptive control of sound radiation from a panel into an acoustic cavity using active constrained layer damping," Smart Materials and Structures, Vol. 5, No. 5, 1996, pp. 649-659.

${ }^{90}$ Illaire, H., A study of active-passive damping treatments, Phd thesis, Department of Applied Acoustics, Chalmers University of Technology, 2004.

${ }^{91}$ Vasques, C. M. A. and Rodrigues, J. D., "Adaptive feedforward control of vibration of a beam with active-passive damping treatments: Numerical analysis and experimental implementation," Proceedings of the 16th International Conference on Adaptive Structures and Technologies (ICAST 2005), DEStech Publications, Paris, France, 2006, pp. 255-262.

${ }^{92}$ Saunders, W. R., Robertshaw, H. H., and Burdisso, R. A., "An evaluation of feedback, adaptive feedforward and hybrid controller designs for active structural control of a lightly-damped structure," 2nd Conference on Recent Advances in Active Control of Sound and Vibration, edited by R. A. Burdisso, Technomic Publishing, Blacksburg, Virginia, 1993, pp. 339-354.

${ }^{93}$ Clark, R. L., "A Hybrid Autonomous Control Approach," Journal of Dynamic Systems Measurement and Control, Vol. 117, No. 2, 1995, pp. 232-240.

${ }^{94}$ Saunders, W. R., Robertshaw, H. H., and Burdisso, R. A., "A hybrid structural control approach for narrow-band and impulsive disturbance rejection," Noise Control Engineering Journal, Vol. 44, No. 1, 1996, pp. 11-21.

${ }^{95}$ Man, P. D. and Preumont, A., "Hybrid Feedback-Feedforward Control for Vibration Suppression," Journal of Structural Control, Vol. 3, No. 1-2, 1996, pp. 33-44.

${ }^{96}$ Vasques, C. M. A. and Rodrigues, J. D., "Simulation of Combined Feedback/Feedforward Active Control of Vibration of Beams with ACLD Treatments," Computers and Structures, 2006 (to be published).

${ }^{97}$ Ambartsumian, S. A., "On a general theory of anisotropic shells," Journal of Applied Mathematics and Mechanics, Vol. 22, No. 2, 1958, pp. 305-319.

${ }^{98}$ Rao, M. K., Desai, Y. M., and Chitnis, M. R., "Free vibrations of laminated beams using mixed theory," Composite Structures, Vol. 52, No. 2, 2001, pp. 149-160.

${ }^{99}$ Rao, M. K. and Desai, Y. M., "Analytical solutions for vibrations of laminated and sandwich plates using mixed theory," Composite Structures, Vol. 63, No. 3-4, 2004, pp. 361-373.

${ }^{100}$ Rao, M. K., Scherbatiuk, K., Desai, Y. M., and Shah, A. H., "Natural vibrations of laminated and sandwich plates," Journal of Engineering Mechanics, Vol. 130, No. 11, 2004, pp. 1268-1278.

${ }^{101}$ Chattopadhyay, A., Gu, H. Z., Beri, R., and Nam, C. H., "Modeling segmented active constrained layer damping using hybrid displacement field," AIAA Journal, Vol. 39, No. 3, 2001, pp. 480-486.

${ }^{102}$ Cho, Y. B. and Averill, R. C., "First-order zig-zag sublaminate plate theory and finite element model for laminated composite and sandwich panels," Composite Structures, Vol. 50, No. 1, 2000, pp. 1-15.

${ }^{103}$ Nye, J. F., Physical Properties of Crystals: Their Representation by Tensors and Matrices, Clarendon Press, Oxford, 1st ed., 1985 .

${ }^{104}$ IEEE, IEEE Standard on Piezoelectricity, ANSI/IEEE Std 176-1987, 1988. 

2003.

${ }^{105}$ Weisstein, E. W., CRC Concise Encyclopedia of Mathematics, Chapman \& Hall / CRC, Boca Raton, CA, 2nd ed.,

${ }^{106}$ Carrera, E., "Transverse normal strain effects on thermal stress analysis of homogeneous and layered plates," AIAA Journal, Vol. 43, No. 10, 2005, pp. 2232-2242.

${ }^{107}$ Reddy, J. N., An Introduction to the Finite Element Method, McGraw-Hill, 2nd ed., 1993.

${ }^{108}$ Kidder, R. L., "Reduction of Structural Frequency Equations," AIAA Journal, Vol. 11, No. 6, 1973, pp. $892-892$.

${ }^{109}$ O'Callahan, J., "A Procedure for an Improved Reduced System (IRS) Model," Proceedings of the 7th International Modal Analysis Conference (IMAC-VII), Society for Experimental Mechanics, Las Vegas, Nevada, 1989, pp. 7-21.

${ }^{110}$ Gordis, J. H., "Analysis of the Improved Reduced System (IRS) Model Reduction Procedure," Modal Analysis: The International Journal of Analytical and Experimental Modal Analysis, Vol. 9, No. 4, 1994, pp. 269-285.

${ }^{111}$ Vasques, C. M. A., Modelização do Controlo Activo de Vibrações de Vigas com Sensores e Actuadores Piezoeléctricos, MSc thesis, Departamento de Engenharia Mecânica e Gestão Industrial, Faculdade de Engenharia da Universidade do Porto, Porto, Portugal, 2003 (in Portuguese).

${ }^{112}$ Vasques, C. M. A., Mace, B. R., Gardonio, P., and Rodrigues, J. D., "Arbitrary Active Constrained Layer Damping Treatments on Beams: Finite Element Modelling and Experimental Validation," Computers and Structures, 2005 (to be published).

${ }^{113}$ Vasques, C. M. A., Mace, B., Gardonio, P., and Rodrigues, J. D., "Analytical Formulation and Finite Element Modelling of Beams with Arbitrary Active Constrained Layer Damping Treatments," Institute of Sound and Vibration Research, Technical Memorandum, TM934, 2004.

${ }^{114}$ Lesieutre, G. A., "Finite Elements for Dynamic Modeling of Uniaxial Rods with Frequency-Dependent Material Properties," International Journal of Solids and Structures, Vol. 29, No. 12, 1992, pp. 1567-1579.

${ }^{115}$ Christensen, R. M., Theory of Viscoelasticity: An Introduction, Academic Press, New York, 2nd ed., 1982.

${ }^{116}$ Burl, J. B., Linear Optimal Control, Addison-Wesley, California, 1999.

${ }^{117}$ Kwakernaak, H. and Sivan, R., Linear Optimal Control Systems, John Wiley \& Sons, New York, 1972.

${ }^{118}$ Lam, M. J., Inman, D. J., and Saunders, W. R., "Hybrid damping models using the Golla-Hughes-McTavish method with internally balanced model reduction and output feedback," Smart Materials and Structures, Vol. 9, No. 3, 2000, pp. 362.

${ }^{119}$ Friswell, M. I. and Inman, D. J., "Reduced-order models of structures with viscoelastic components," AIAA Journal, Vol. 37, No. 10, 1999, pp. 1318-1325.

${ }^{120}$ Park, C. H., Inman, D. J., and Lam, M. J., "Model Reduction of Viscoelastic Finite Element Models," Journal of Sound and Vibration, Vol. 219, No. 4, 1999, pp. 619-637.

${ }^{121}$ Bouc, R. and Friot, E., "Contrôle Optimal par Retroaction du Rayonnement d'une Plaque Munie de Capteurs et d'Actionneurs Piézo-électriques non Colocalisés," 2eme Colloque GDR Vibroacoustique, Marseille, 1996, pp. 229-248.

${ }^{122}$ Widrow, B. and Stearns, S. D., Adaptive Signal Processing, Prentice-Hall, Englewood Cliffs, 1985.

${ }^{123}$ Haykin, S. S., Adaptive Filter Theory, Prentice Hall, Upper Saddle River, NJ, 4th ed., 2001.

${ }^{124}$ Sokolnikoff, I. S., Mathematical Theory of Elasticity, McGraw-Hill, New York, NY, 2nd ed., 1956. 